\title{
Foncteur de Picard d'un champ algébrique
}

\author{
Sylvain Brochard
}

Received: 24 October 2007 / Published online: 23 September 2008

(C) The Author(s) 2008. This article is published with open access at Springerlink.com

Résumé In this article we study the Picard functor and the Picard stack of an algebraic stack. We give a new and direct proof of the representability of the Picard stack. We prove that it is quasi-separated, and that the connected component of the identity is proper when the fibers of $\mathscr{X}$ are geometrically normal. We study some examples of Picard functors of classical stacks. In an appendix, we review the lisse-étale cohomology of abelian sheaves on an algebraic stack.

\section{Table des matières}

1 Introduction . . . . . . . . . . . . . . . . . . . . . . . . . . 542

2 Foncteurs de Picard et champ de Picard . . . . . . . . . . . . . . . . . . . . . . . . . . . . . . . . 544

2.1 Description cohomologique . . . . . . . . . . . . . . . . . . . . . . 544

2.2 Comparaison entre les différents foncteurs de Picard . . . . . . . . . . . . . . . . . . . . . 546

2.3 Le champ de Picard d'un champ algébrique . . . . . . . . . . . . . . . . . . . . . . . 548

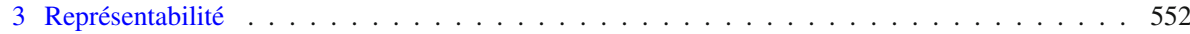

3.1 Un champ algébrique au sens d'Artin . . . . . . . . . . . . . . . . . . . . . . . . . . . . . . . . . 552

3.2 Quasi-séparation et séparation . . . . . . . . . . . . . . . . . . 5 558

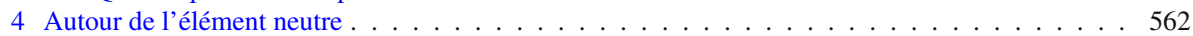

4.1 Lissité et dimension . . . . . . . . . . . . . . . . . . . . . . . . . 562

4.2 Construction de la composante neutre . . . . . . . . . . . . . . . . . . . . . . . . . . . . . . . . . . . . . . . 564

4.3 Propreté de la composante neutre . . . . . . . . . . . . . . . . . . . . . . . 569

5 Quelques exemples . . . . . . . . . . . . . . . . . . . . . . . 5 574

5.1 Espace de module des courbes elliptiques . . . . . . . . . . . . . . . . . . . . 574

5.2 Espaces projectifs à poids . . . . . . . . . . . . . . . . . . . . . . . . . 575

5.3 Racine $n^{\text {ième }}$ d'un faisceau inversible . . . . . . . . . . . . . . . . . . . . . 577

5.4 Courbes tordues d'Abramovich et Vistoli . . . . . . . . . . . . . . . . . . . . . . 583

S. Brochard $(\varangle)$

Mathematisch Instituut, Universiteit Leiden, Postbus 9512,

2300 RA Leiden, The Netherlands

e-mail: brochard@math.leidenuniv.nl 
A Cohomologie lisse-étale sur les champs algébriques . . . . . . . . . . . . . . . . . . . 587

A.1 Topos lisse-étale et cohomologie des faisceaux . . . . . . . . . . . . . . . . . . . . 587

A.2 Suites spectrales . . . . . . . . . . . . . . . . . . . . . . . . . 591

A.3 Comparaison de cohomologies . . . . . . . . . . . . . . . . . . 593

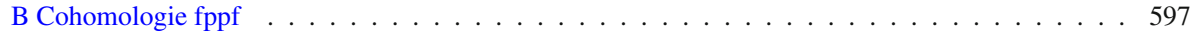

B.1 Sorites sur la cohomologie plate . . . . . . . . . . . . . . . . . . . 597

B.2 Comparaison avec la cohomologie lisse-étale . . . . . . . . . . . . . . . . . . . . . 599

\section{Introduction}

L'objet principal de cet article est l'étude du foncteur de Picard et du champ de Picard d'un champ algébrique. Notre but est de généraliser au cas des champs algébriques les résultats classiques valables dans le cadre des schémas, que l'on peut trouver dans [31] ou [14].

Si $\mathscr{X}$ est un champ algébrique sur un schéma $S$, on appelle champ de Picard de $\mathscr{X}$, et l'on note $\mathscr{P} i c(\mathscr{X} / S)$, le champ classifiant des faisceaux inversibles sur $\mathscr{X}$, i.e. le champ dont la catégorie fibre en $U$ est la catégorie des faisceaux inversibles sur $\mathscr{X} \times{ }_{S} U$. On définit aussi le foncteur de Picard relatif de la manière suivante. On commence par définir un foncteur $P_{\mathscr{X} / S}$ qui à tout $U$ associe

$$
P_{\mathscr{X} / S}(U)=\frac{\operatorname{Pic}\left(\mathscr{X} \times{ }_{S} U\right)}{\operatorname{Pic}(U)} .
$$

On note alors Pic $\mathscr{X} / S$ (Zar) (resp. Pic $\mathscr{X}_{/ S}$ (Ét) , Pic $\mathscr{X} / S$ (fppf) $)$ le faisceau associé à $P_{\mathscr{X} / S}$ pour la topologie de Zariski (resp. étale, fppf) ${ }^{1}$. Nous démêlons dans la première partie les liens étroits qui unissent les différents foncteurs de Picard et le champ de Picard.

La première question qui se pose est celle de l'algébricité du champ de Picard (donc par 2.3.3 celle de la représentabilité du foncteur de Picard). Elle a été résolue par Aoki.

Théorème 1.1 Aoki [11, 5.1], [10] Si $\mathscr{X}$ est propre, plat et de présentation finie sur $S$, alors le champ $\mathscr{P} i c(\mathscr{X} / S)$ est un champ algébrique au sens d'Artin.

Cependant ce résultat apparaît comme un cas particulier d'un théorème plus général et plus difficile. Aoki montre en effet dans [11] que si $\mathscr{X}$ et $\mathscr{Y}$ sont deux champs algébriques, alors sous de bonnes hypothèses le champ $\mathscr{H}$ om $(\mathscr{X}, \mathscr{Y})$ est un champ algébrique au sens d'Artin. La démonstration fait appel à un certain nombre de résultats non triviaux concernant les déformations de morphismes d'espaces algébriques [30], les déformations de morphismes représentables de champs algébriques [42], les déformations de champs algébriques [9] et la théorie du complexe cotangent [30,34, 40]. Le cas du champ de Picard s'en déduit en prenant pour $\mathscr{Y}$ le champ B $\mathbb{G}_{\mathrm{m}}$. Nous en donnons au paragraphe 3.1 une preuve directe, plus rapide et plus concrète.

Nous traitons au paragraphe 3.2 la question de la quasi-séparation. Le résultat principal est le suivant:

\footnotetext{
${ }_{1}^{1}$ Lorsque nous parlerons $d u$ foncteur de Picard de $\mathscr{X} / S$, il s'agira de Pic $\mathscr{X} / S$ (fppf). Nous le noterons Pic $\mathscr{X} / S$ s'il n'y a pas d'ambiguïté.
} 
Théorème 1.2 Soient $S$ un schéma localement noethérien, et $\mathscr{X}$ un S-champ algébrique propre, plat, et cohomologiquement plat en dimension zéro. Alors le champ de Picard $\mathscr{P}$ ic $(\mathscr{X} / S)$ est quasi-séparé (donc est algébrique au sens de [34]).

Nous étudions ensuite la composante neutre du foncteur de Picard. Le résultat des EGA sur la composante connexe des fibres le long d'une section [29, (15.6.5)] que l'on utilise usuellement pour la définir ne s'applique pas tel quel lorsque Pic $\mathscr{X} / S$ est un espace algébrique. Notre premier travail a donc été de généraliser ce résultat au cas des espaces algébriques afin de s'assurer que la construction habituelle fournirait bien un sous-espace algébrique ouvert $\operatorname{Pic}_{\mathscr{X} / S}^{0}$ du foncteur de Picard.

Une fois la composante neutre définie, nous nous posons la question de sa propreté sur $S$. Nous aboutissons au résultat suivant, qui généralise les résultats analogues pour les schémas.

Théorème 1.3 Soit $\mathscr{X}$ un S-champ algébrique propre et plat. On suppose que $\mathscr{X}$ est à fibres géométriquement normales et géométriquement intègres, et que $\mathrm{Pic}_{\mathscr{X}}^{0} / S$ est un ouvert de $\operatorname{Pic} \mathscr{X} / S$ de type fini sur S. Alors Pic $\mathscr{X} / S$ est un $S$-espace algébrique séparé et $\mathrm{Pic}_{\mathscr{X} / S}^{0}$ est propre sur $S$.

Nous montrons d'abord le théorème sur un corps ( $c f$. 4.3.1). La démonstration repose sur une étude des $\mathbb{Z}$-torseurs sur un champ normal : nous commençons par montrer que si $\mathscr{X}$ est un champ algébrique localement noethérien et normal alors le groupe $H^{1}(\mathscr{X}, \mathbb{Z})$ est trivial.

Enfin, nous illustrons le texte en étudiant le foncteur de Picard de quelques champs algébriques classiques : l'espace de modules des courbes elliptiques, les espaces projectifs à poids, le champ des racines $n^{\text {ièmes }}$ d'un faisceau inversible, et les courbes tordues d'Abramovich et Vistoli.

\section{REVUE DE LA COHOMOLOGIE LISSE- ÉTALE}

Le défaut de fonctorialité du topos lisse-étale des champs algébriques est maintenant bien connu : si $f: \mathscr{X} \rightarrow \mathscr{Y}$ est un morphisme de champs algébriques, le foncteur $f^{-1}$ n'est pas toujours exact. Ceci a pour conséquence fâcheuse que la « machine » SGA4 ne s'applique pas toujours et qu'il faut par conséquent travailler un peu plus finement pour obtenir un certain nombre de propriétés d'apparence pourtant élémentaire sur la cohomologie lisse-étale des champs algébriques. Ce travail a été fait en grande partie par Olsson et Laszlo pour le cas des faisceaux quasi-cohérents (voir [40]) ou des coefficients finis (voir [33]). Mais les faisceaux abéliens qui ne jouissent pas d'une telle structure ont pour l'instant été laissés de côté.

Or le faisceau abélien que l'on rencontre le plus souvent lorsque l'on s'intéresse au foncteur de Picard n'est pas quasi-cohérent : il s'agit de $\mathbb{G}_{\mathrm{m}}$. Nous avons donc été amené à démontrer au fil de nos travaux diverses propriétés. Leur nombre croissant nous a finalement conduit à les regrouper dans un chapitre à part. Cette annexe est donc une sorte de «fourre-tout » cohomologique qui, bien loin de prétendre à l'exhaustivité, se contente au contraire de répondre aux strictes exigences des autres chapitres. Nous espérons tout de même que nous aurons ainsi contribué à combler une lacune de la littérature existante.

Je remercie chaleureusement Laurent Moret-Bailly pour la patience et la disponibilité dont il a su faire preuve dans son travail de direction, et pour les relectures 
minutieuses de ma thèse, dont cet article est issu. Je remercie également le rapporteur pour ses nombreuses remarques et corrections, qui ont incontestablement amélioré la qualité de l'article.

\section{Conventions}

Suivant [34], sauf mention expresse du contraire, tous les champs algébriques (a fortiori tous les schémas et tous les espaces algébriques) seront quasi-séparés. Un champ «algébrique » non-quasi-séparé, i.e. un champ dont la diagonale est représentable et localement de type fini, et qui admet une présentation lisse, sera appelé «champ algébrique au sens d'Artin ». Nous renvoyons aux annexes pour le formalisme cohomologique. Nous signalons simplement que, sauf mention explicite du contraire, nous travaillons toujours avec le site lisse-étale des champs algébriques.

\section{Foncteurs de Picard et champ de Picard}

\subsection{Description cohomologique}

Comme dans le cas des schémas, on peut calculer le groupe de Picard de $\mathscr{X}$ à l'aide de la cohomologie de $\mathbb{G}_{\mathrm{m}}$. Rappelons tout d'abord le résultat de descente ci-dessous.

Proposition 2.1.1 [34, (13.5)] Soit $f: \mathscr{Y} \rightarrow \mathscr{X}$ un morphisme fidèlement plat de champs algébriques, que l'on suppose de plus quasi-compact ou localement de présentation finie. Alors la catégorie des faisceaux inversibles sur $\mathscr{X}$ est équivalente à la catégorie $\operatorname{Desc}(\mathscr{Y}, \mathscr{X})$ décrite de la manière suivante. Un objet est un couple $(\mathscr{L}, \alpha)$ où $\mathscr{L}$ est un faisceau inversible sur $\mathscr{Y}$ et $\alpha: p_{1}^{*} \mathscr{L} \rightarrow p_{2}^{*} \mathscr{L}$ est un isomorphisme tel que, à des isomorphismes canoniques près, $\left(p_{23}^{*} \alpha\right) \circ\left(p_{12}^{*} \alpha\right)=p_{13}^{*} \alpha$. Un morphisme de $(\mathscr{L}, \alpha)$ vers $(\mathscr{M}, \beta)$ est un morphisme $\gamma: \mathscr{L} \rightarrow \mathscr{M}$ tel que $\left(p_{2}^{*} \gamma\right) \circ \alpha=\beta \circ\left(p_{1}^{*} \gamma\right)$.

Proposition 2.1.2 Soit $\mathscr{X}$ un S-champ algébrique. Alors:

$$
\operatorname{Pic} \mathscr{X} \simeq H^{1}\left(\mathscr{X}, \mathbb{G}_{\mathrm{m}}\right)
$$

où $H^{1}\left(\mathscr{X}, \mathbb{G}_{\mathrm{m}}\right)$ est le premier groupe de cohomologie du faisceau $\mathbb{G}_{\mathrm{m}}$ sur $\mathscr{X}$ muni de la topologie lisse-étale calculé au sens des foncteurs dérivés.

Démonstration. Soit $T \rightarrow \mathscr{X}$ une présentation de $\mathscr{X}$. On reprend ici les notations de l'annexe A.2 qui précèdent le théorème A.2.1. On considère en particulier le premier groupe de cohomologie « à la Čech » associé à cette présentation :

$$
\check{H}^{1}\left(H^{0}\left(T^{\bullet}, \mathbb{G}_{\mathrm{m}}\right)\right)=\frac{\operatorname{Ker}\left(p_{23}^{*}-p_{13}^{*}+p_{12}^{*}\right)}{\operatorname{Im}\left(p_{1}^{*}-p_{2}^{*}\right)} .
$$

Se donner un 1-cocycle de Čech à valeurs dans $\mathbb{G}_{\mathrm{m}}$ revient à se donner une donnée de descente sur $\mathscr{O}_{T}$, et deux telles données de descente $g_{1}, g_{2}$ définissent le même élément dans $\check{H}^{1}\left(H^{0}\left(T^{\bullet}, \mathbb{G}_{\mathrm{m}}\right)\right)$ si et seulement si $\left(\mathscr{O}_{T}, g_{1}\right)$ et $\left(\mathscr{O}_{T}, g_{2}\right)$ sont isomorphes 
dans la catégorie des faisceaux inversibles sur $T$ munis d'une donnée de descente relativement à $T \rightarrow \mathscr{X}$. Compte tenu de l'équivalence 2.1 .1 entre cette catégorie et la catégorie des faisceaux inversibles sur $\mathscr{X}$, le groupe $\check{H}^{1}\left(H^{0}\left(T^{\bullet}, \mathbb{G}_{\mathrm{m}}\right)\right)$ s'identifie à l'ensemble des classes d'isomorphie de faisceaux inversibles sur $\mathscr{X}$ dont l'image inverse sur $T$ est isomorphe à $\mathscr{O}_{T}$, donc au groupe $\operatorname{Ker}(\operatorname{Pic}(\mathscr{X}) \rightarrow \operatorname{Pic}(T))$. De plus cet isomorphisme est fonctoriel en $T$. En passant à la limite inductive, on obtient un isomorphisme

$$
\underset{\lim }{\longrightarrow} \check{H}^{1}\left(H^{0}\left(T^{\bullet}, \mathbb{G}_{\mathrm{m}}\right)\right) \simeq \operatorname{Pic} \mathscr{X}
$$

où la limite inductive est prise sur l'ensemble des présentations lisses $T \rightarrow \mathscr{X}$ de $\mathscr{X}$. On dispose par ailleurs de la suite exacte en bas degrés associée à la suite spectrale de descente A.2.1 :

$$
0 \rightarrow \check{H}^{1}\left(H^{0}\left(T^{\bullet}, \mathbb{G}_{\mathrm{m}}\right)\right) \rightarrow H^{1}\left(\mathscr{X}, \mathbb{G}_{\mathrm{m}}\right) \rightarrow \check{H}^{0}\left(H^{1}\left(T^{\bullet}, \mathbb{G}_{\mathrm{m}}\right)\right) .
$$

Cette suite exacte étant fonctorielle en $T$, on voit que les injections des $\check{H}^{1}\left(H^{0}\left(T^{\bullet}\right.\right.$, $\left.\left.\mathbb{G}_{\mathrm{m}}\right)\right)$ dans $H^{1}\left(\mathscr{X}, \mathbb{G}_{\mathrm{m}}\right)$ induisent un morphisme injectif de $\lim \check{H}^{1}\left(H^{0}\left(T^{\bullet}, \mathbb{G}_{\mathrm{m}}\right)\right)$ dans $H^{1}\left(\mathscr{X}, \mathbb{G}_{\mathrm{m}}\right)$. Pour conclure, il ne reste plus qu'à montrer, vu la suite exacte en bas degrés ci-dessus, que pour tout $x \in H^{1}\left(\mathscr{X}, \mathbb{G}_{\mathrm{m}}\right)$, il existe une présentation $T \rightarrow \mathscr{X}$ telle que l'image de $x$ dans $H^{1}\left(T, \mathbb{G}_{\mathrm{m}}\right)$ soit nulle. C'est un exercice facile laissé au lecteur.

On peut calculer les groupes de Picard relatifs à l'aide des images directes supérieures de $\mathbb{G}_{\mathrm{m}}$.

Proposition 2.1.3 Soit $\mathscr{X}$ un $S$-champ algébrique. On note $f: \mathscr{X} \rightarrow S$ le morphisme structural. Alors pour tout S-schéma $T$ :

$$
\begin{aligned}
& \operatorname{Pic}_{\mathscr{X} / S(\text { Ét })}(T) \simeq H^{0}\left(T, R^{1} f_{T *} \mathbb{G}_{\mathrm{m}}\right) \\
& \operatorname{Pic}_{\mathscr{X} / S(\text { fppf })}(T) \simeq H_{\mathrm{pl}}^{0}\left(T, R^{1} f_{T *}^{\mathrm{pl}} \mathbb{G}_{\mathrm{m}}\right) \text {. }
\end{aligned}
$$

Démonstration. D'après la proposition A.2.5, la restriction au site étale de $T$ du faisceau $R^{1} f_{T *} \mathbb{G}_{\mathrm{m}}$ est le faisceau étale associé à

$$
U \longmapsto H^{1}\left(\mathscr{X}_{T} \times_{T} U, \mathbb{G}_{\mathrm{m}}\right)=\operatorname{Pic}\left(\mathscr{X}_{T} \times_{T} U\right)
$$

Donc $\left(R^{1} f_{T *} \mathbb{G}_{\mathrm{m}}\right)$ ét $=\operatorname{Pic}_{\mathscr{X}_{T} / T \text { (Ét) }}$ et en particulier

$$
H^{0}\left(T, R^{1} f_{T *} \mathbb{G}_{\mathrm{m}}\right)=\operatorname{Pic}_{\mathscr{X}_{T} / T(\mathrm{E} t)}(T)=\operatorname{Pic}_{\mathscr{X} / S(\text { Ét) }}(T) .
$$

On obtient la seconde assertion en appliquant exactement le même raisonnement pour la topologie fppf. On utilise B.1.4 au lieu de A.2.5. Le groupe $H_{\mathrm{pl}}^{1}\left(\mathscr{X}_{T} \times_{T} U, \mathbb{G}_{\mathrm{m}}\right)$ est isomorphe à $\operatorname{Pic}\left(\mathscr{X}_{T} \times_{T} U\right)$ par B.2.5. 


\subsection{Comparaison entre les différents foncteurs de Picard}

Remarque 2.2.1 On peut d'ores et déjà comparer les groupes de sections des différents foncteurs de Picard dans quelques cas particuliers grâce à la remarque de topologie suivante. Supposons que $U$ soit un $S$-schéma affine tel que, pour toute famille couvrante $\left(U_{i} \rightarrow U\right)_{i \in I}$ pour une certaine topologie (Top), il existe un indice $i \in I$ tel que le morphisme $U_{i} \rightarrow U$ ait une section. Alors le morphisme $\operatorname{Pic}\left(\mathscr{X} \times{ }_{S} U\right) \rightarrow \operatorname{Pic} \mathscr{X} / S($ Top) $(U)$ est un isomorphisme. En particulier, si $U$ est le spectre d'un anneau local (resp. d'un anneau local strictement hensélien, d'un corps

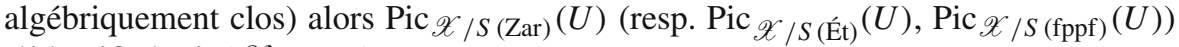
s'identifie à $\operatorname{Pic}\left(\mathscr{X} \times{ }_{S} U\right)$.

La notion de morphisme cohomologiquement plat en dimension zéro se généralise sans difficultés aux champs algébriques.

Définition 2.2.2 On dit qu'un morphisme de champs algébriques $f: \mathscr{X} \rightarrow \mathscr{Y}$ est cohomologiquement plat en dimension zéro s'il est plat et si le morphisme

$$
f^{\sharp}: \mathscr{O}_{\mathscr{Y}} \longrightarrow f_{*} \mathscr{O}_{\mathscr{X}}
$$

est un isomorphisme universellement (en particulier $\mathscr{X}$ et $\mathscr{Y}$ ont alors les mêmes sections globales).

Remarque 2.2.3 Attention, la notion de morphisme cohomologiquement plat en dimension zéro apparaît de différentes manières dans la littérature. Par exemple dans $[14,8.1$, p. 206] ou dans [12, 7.2, p. 67], un morphisme de schémas $f: X \rightarrow S$ est dit cohomologiquement plat en dimension zéro si la formation de $f_{*} \mathscr{O}_{X}$ commute au changement de base. La définition adoptée ici est plus forte.

Remarque 2.2.4 Un morphisme cohomologiquement plat en dimension zéro est surjectif. En effet, pour tout point $s: \operatorname{Spec} k \rightarrow \mathscr{Y}$ de $\mathscr{Y}$, la fibre $\mathscr{X}_{s}$ de $\mathscr{X}$ au-dessus de $s$ est non vide puisque $\Gamma\left(\mathscr{X}_{s}, \mathscr{O}_{\mathscr{X}_{s}}\right)$ est isomorphe à $k$. On en déduit que si $S$ est un schéma et $f: \mathscr{X} \rightarrow S$ un morphisme localement de présentation finie et cohomologiquement plat en dimension zéro de $S$-champs algébriques alors $f$ a une section localement pour la topologie (fppf) sur $S$. En effet, soit $S^{\prime} \rightarrow \mathscr{X}$ une présentation de $\mathscr{X}$. Alors le morphisme $S^{\prime} \rightarrow S$ est couvrant pour la topologie $f p p f$, et il est clair que le morphisme induit par $f$ sur $S^{\prime}$ a une section.

Remarque 2.2.5 La notion de platitude cohomologique est intimement liée à la connexité des fibres géométriques. En effet si $\mathscr{X}$ est cohomologiquement plat en dimension zéro sur $\mathscr{Y}$, ses fibres sont géométriquement connexes. Réciproquement, dans le cas où le morphisme $f$ est propre et plat, il suffit que les fibres de $\mathscr{X}$ soient géométriquement connexes et géométriquement réduites pour que $\mathscr{X}$ soit cohomologiquement plat en dimension zéro sur $\mathscr{Y}$ (au moins lorsque $\mathscr{Y}$ est noethérien et réduit).

Le théorème suivant permet de comparer les différents foncteurs de Picard d'un champ algébrique. Il généralise [31, 2.5]. 
Théorème 2.2.6 Soit $f: \mathscr{X} \rightarrow S$ un $S$-champalgébrique cohomologiquement plat en dimension zéro. Alors les morphismes naturels

$$
P_{\mathscr{X}} / S \stackrel{i_{1}}{\longrightarrow} \operatorname{Pic} \mathscr{X} / S(\text { Zar }) \stackrel{i_{2}}{\longrightarrow} \text { Pic } \mathscr{X} / S(\text { Ét })
$$

sont injectifs, et le morphisme naturel

$$
\operatorname{Pic}_{\mathscr{X} / S(\mathrm{E} t)} \longrightarrow \operatorname{Pic} \mathscr{X} / S(\mathrm{fppf})
$$

est un isomorphisme. Si de plus $f$ a une section localement pour la topologie de Zariski, alors $i_{2}$ est un isomorphisme. Enfin si $f$ a une section, $i_{1}$ est lui aussi un isomorphisme.

Démonstration. Soit $T$ un $S$-schéma. La suite spectrale de Leray

$$
H^{p}\left(T, R^{q} f_{T *} \mathbb{G}_{\mathrm{m}}\right) \Rightarrow H^{p+q}\left(\mathscr{X}_{T}, \mathbb{G}_{\mathrm{m}}\right)
$$

induit la suite exacte longue en bas degrés suivante:

$$
\begin{aligned}
0 & \rightarrow H^{1}\left(T, f_{T *} \mathbb{G}_{\mathrm{m}}\right) \rightarrow H^{1}\left(\mathscr{X}_{T}, \mathbb{G}_{\mathrm{m}}\right) \rightarrow H^{0}\left(T, R^{1} f_{T *} \mathbb{G}_{\mathrm{m}}\right) \rightarrow \\
& \rightarrow H^{2}\left(T, f_{T *} \mathbb{G}_{\mathrm{m}}\right) \rightarrow H^{2}\left(\mathscr{X}_{T}, \mathbb{G}_{\mathrm{m}}\right)
\end{aligned}
$$

(où tous les calculs sont effectués pour la topologie lisse-étale sur le champ algébrique considéré. En particulier lorsqu'il s'agit d'un schéma, cela revient d'après A.1.6 à calculer sa cohomologie pour la topologie étale.) Le morphisme $f$ étant cohomologiquement plat en dimension zéro, on a $f_{T *} \mathbb{G}_{\mathrm{m}}=\mathbb{G}_{\mathrm{m}}$. D'après les propriétés précédentes, le début de la suite exacte ci-dessus fournit la suite exacte:

$$
0 \rightarrow \operatorname{Pic}(T) \rightarrow \operatorname{Pic}\left(\mathscr{X}_{T}\right) \rightarrow \operatorname{Pic}_{\mathscr{X} / S(\mathrm{E} t)}(T)
$$

ce qui montre que le morphisme naturel $P_{\mathscr{X} / S} \rightarrow \mathrm{Pic}_{\mathscr{X} / S \text { (Ét) }}$ est injectif. Il en résulte, d'une part, que le morphisme naturel $P_{\mathscr{X} / S} \rightarrow \mathrm{Pic}_{\mathscr{X}} / S$ (Zar) est injectif, et d'autre part, en appliquant le foncteur «faisceau associé pour la topologie de Zariski », que le morphisme naturel Pic $\mathscr{X} / S$ (Zar) $\rightarrow$ Pic $_{\mathscr{X} / S \text { (Ét) }}$ est injectif.

Dans le cas où $f$ a une section, le morphisme induit par $f$ de $H^{2}\left(T, f_{T *} \mathbb{G}_{\mathrm{m}}\right)$ vers $H^{2}\left(\mathscr{X}_{T}, \mathbb{G}_{\mathrm{m}}\right)$ a une rétraction, donc il est injectif. Mais alors la flèche de $H^{0}\left(T, R^{1} f_{T *} \mathbb{G}_{\mathrm{m}}\right)$ vers $H^{2}\left(T, f_{T *} \mathbb{G}_{\mathrm{m}}\right)$ est nulle, et donc $H^{1}\left(\mathscr{X}_{T}, \mathbb{G}_{\mathrm{m}}\right) \rightarrow H^{0}$ $\left(T, R^{1} f_{T *} \mathbb{G}_{\mathrm{m}}\right)$ est surjectif, d'où une suite exacte courte:

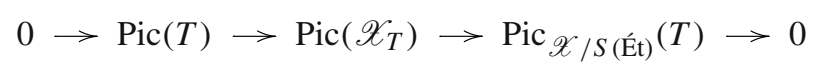

ce qui montre que $i_{1}$ et $i_{2}$ sont des isomorphismes.

L'avant-dernière assertion en découle immédiatement puisque la question de savoir si $i_{2}$ est un isomorphisme est locale pour la topologie de Zariski. 


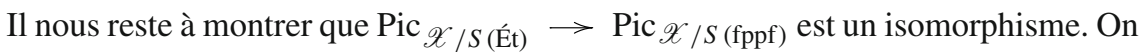
a un diagramme commutatif dans lequel les lignes sont les suites exactes de bas degré associées aux suites spectrales de Leray pour la cohomologie lisse-étale et pour la cohomologie fppf :

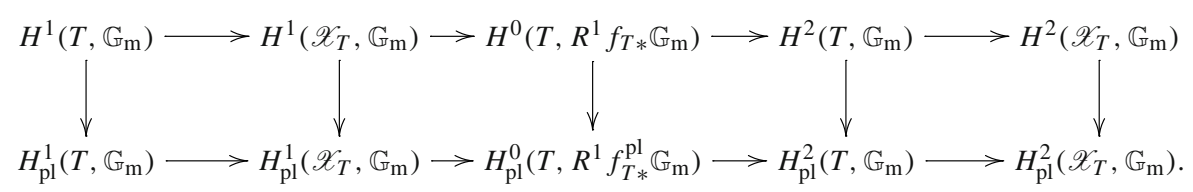

Le diagramme ci-dessus tient compte du fait que, par platitude cohomologique, $f_{T *} \mathbb{G}_{\mathrm{m}}=\mathbb{G}_{\mathrm{m}}$ et $f_{T *}^{\mathrm{pl}} \mathbb{G}_{\mathrm{m}}=\mathbb{G}_{\mathrm{m}}$. Comme $\mathbb{G}_{\mathrm{m}}$ est un groupe lisse sur la base, le théorème B.2.5 nous dit que les deux flèches verticales de gauche et les deux flèches verticales de droite sont des isomorphismes. D'après le lemme des 5 , celle du milieu en est un aussi. Or d'après 2.1.3 cette flèche est précisément le morphisme $\operatorname{Pic}_{\mathscr{X} / S(\text { Ét) }}(T) \rightarrow \operatorname{Pic} \mathscr{X}_{/ S(\text { fppf })}(T)$.

\subsection{Le champ de Picard d'un champ algébrique}

Avec l'apparition des champs, un nouvel objet «classifiant» pour les faisceaux inversibles est venu s'ajouter aux cinq foncteurs précédents : le champ de Picard. Il résulte aisément de 2.1 .1 que le $S$-groupoïde $\mathscr{P}$ ic $(\mathscr{X} / S)$ défini en introduction est bien un $S$-champ. De plus la formation de $\mathscr{P} i c(\mathscr{X} / S)$ commute au changement de base. Par ailleurs, en notant $\mathscr{H}$ om $(\mathscr{X}, \mathscr{Y})$ le $S$-champ dont la fibre en $U$ est la catégorie $\operatorname{Hom}\left(\mathscr{X} \times{ }_{S} U, \mathscr{Y} \times{ }_{S} U\right)$, il est clair que $\mathscr{P} i c(\mathscr{X} / S)$ est isomorphe à $\mathscr{H}$ om $\left(\mathscr{X}, \mathrm{B} \mathbb{G}_{\mathrm{m}}\right)$. La proposition 2.3.3 et le corollaire 2.3.6 montrent que dans le cas cohomologiquement plat, les questions de l'algébricité du champ de Picard et de la représentabilité du foncteur de Picard sont essentiellement équivalentes.

Remarque 2.3.1 La terminologie adoptée ici, qui semble s'imposer naturellement, n'entre pas en conflit avec celle de [34, (14.4.2)], où l'on appelle « champ de Picard» un champ muni d'un morphisme d' addition qui en fait une sorte de « champ en groupes ». En effet, si $\mathscr{X}$ est un $S$-champ, le champ de Picard de $\mathscr{X}$ défini ci-dessus est bien un champ de Picard au sens de [34, (14.4.2)], le 1-morphisme d'addition

$$
+: \mathscr{P}_{i c}(\mathscr{X} / S) \times_{S} \mathscr{P}_{i c}(\mathscr{X} / S) \longrightarrow \mathscr{P} i c(\mathscr{X} / S)
$$

étant donné par le produit tensoriel de faisceaux inversibles.

Le champ $\mathscr{P}$ ic $(\mathscr{X} / S)$ est muni d'un morphisme naturel vers le $S$-groupoïde associé au préfaisceau $P_{\mathscr{X} / S}$, défini sur $\mathscr{P} i c(\mathscr{X} / S)_{U}$ par le foncteur qui envoie un faisceau inversible sur sa classe d'isomorphie dans $P_{\mathscr{X} / S}(U)$. On en déduit par composition un morphisme naturel $\pi_{\text {ét }}\left(\right.$ resp. $\left.\pi_{f p p f}\right)$ de $\mathscr{P}_{i c}(\mathscr{X} / S)$ vers le $S$-espace Pic $\mathscr{X} / S$ (Ét) (resp. Pic $\mathscr{X} / S$ (fppf) ). Il sera noté $\pi$ lorsque Pic $\mathscr{X} / S$ (Ét) et Pic $\mathscr{X} / S$ (fppf) coïncident (par exemple dans le cas cohomologiquement plat). 
Proposition 2.3.2 Le morphisme de $S$-champs $\pi_{\text {ét }}$ (resp. $\pi_{f p p f}$ ) est une gerbe (resp. une gerbe (fppf)), autrement dit c'est un épimorphisme étale (resp. fppf) et son morphisme diagonal aussi.

Démonstration. Notons $P$ le $S$-espace Pic $\mathscr{X} / S$ (Ét) $($ resp. Pic $\mathscr{X} / S$ (fppf) $)$. Soient $U \in$ ob (Aff/S) et $x \in P(U)$. Il existe par définition de $P$ une famille couvrante (pour la topologie considérée) dans (Aff/S), que l'on peut supposer réduite à un élément $\left(U^{\prime} \rightarrow U\right)$, et un élément $l \in P_{\mathscr{X} / S}\left(U^{\prime}\right)$, tels que l'image de $l$ dans $P\left(U^{\prime}\right)$ soit égale à $x_{\mid U^{\prime}}$. En d'autres termes il existe un faisceau inversible $\mathscr{L}$ dans $\mathscr{P}$ ic $(\mathscr{X} / S)_{U^{\prime}}$ dont l'image dans $P\left(U^{\prime}\right)$ est $x_{\mid U^{\prime}}$, ce qui montre que $\pi_{\text {ét }}$ (resp. $\pi_{f p p f}$ ) est un épimorphisme. Ce n'est pas plus difficile pour le morphisme diagonal.

Proposition 2.3.3 Si $f: \mathscr{X} \rightarrow$ S est cohomologiquement plat, et $\operatorname{si} \mathscr{P}$ ic $(\mathscr{X} / S)$ est un champ algébrique (resp. algébrique au sens d'Artin), alors $\mathrm{Pic} \mathscr{X} / S$ est représentable par un $S$-espace algébrique (resp. algébrique au sens d'Artin), et le 1-morphisme $\pi: \mathscr{P} i c(\mathscr{X} / S) \rightarrow \operatorname{Pic}_{\mathscr{X} / S}$ est fidèlement plat et localement de présentation finie.

Démonstration. D'après le corollaire (10.8) de [34], il suffit de vérifier que pour tout $U \in \mathrm{ob}(\mathrm{Aff} / \mathrm{S})$ et pour tout faisceau inversible $\mathscr{L}$ sur $\mathscr{X} \times{ }_{S} U$, le $U$-espace algébrique en groupes $\mathscr{I}_{\text {som }}(\mathscr{L}, \mathscr{L})$ est plat et localement de présentation finie. Or, par platitude cohomologique, il est isomorphe à $\mathbb{G}_{\mathrm{m}}$.

Réciproquement, on peut essayer de déduire l'algébricité du champ de Picard de celle du foncteur de Picard. Voyons comment. Le champ des faisceaux inversibles sur $S$, c'est-à-dire le champ dont la catégorie fibre au-dessus d'un $S$-schéma $U$ est la catégorie des faisceaux inversibles sur $U$, s'identifie naturellement au champ B $\mathbb{G}_{\mathrm{m}}$ sur $S$. Le foncteur «image inverse par $f$ » induit donc un morphisme de $S$-champs algébriques

$$
\mathrm{B} \mathbb{G}_{\mathrm{m}} \stackrel{f^{*}}{\longrightarrow} \mathscr{P} i c(\mathscr{X} / S) \text {. }
$$

De plus ce morphisme est un monomorphisme (i.e. les foncteurs $f_{U}^{*}$ sont pleinement fidèles) dès que $f$ est cohomologiquement plat en dimension zéro. On a donc dans ce cas une « suite exacte» de champs de Picard:

$$
0 \longrightarrow \mathrm{B} \mathbb{G}_{\mathrm{m}} \stackrel{f^{*}}{\longrightarrow} \operatorname{P} i c(\mathscr{X} / S) \stackrel{\pi}{\longrightarrow} \operatorname{Pic} \mathscr{X} / S \longrightarrow 0 .
$$

Autrement dit $f^{*}$ est un monomorphisme, $\pi$ est un épimorphisme, un objet de $\mathscr{P} i c(\mathscr{X} / S)$ est envoyé sur 0 par $\pi$ si et seulement s'il provient de $\mathrm{B} \mathbb{G}_{\mathrm{m}}$, et tous les morphismes sont compatibles aux morphismes d'addition des champs de Picard considérés $^{2}$. La proposition ci-dessous nous permet de déterminer quand cette suite exacte est scindée.

\footnotetext{
2 Si $f$ n'est pas cohomologiquement plat en dimension zéro, $f^{*}$ n'est plus un monomorphisme, mais le reste est presque vrai : il faut juste remplacer «provient » par «provient localement pour la topologie étale ».
} 
Proposition 2.3.4 Soit $\mathscr{X}$ un S-champ algébrique cohomologiquement plat en dimension zéro.

(i) Les propositions suivantes sont équivalentes:

a) La suite exacte ci-dessus est scindée, autrement dit il existe un morphisme $s$ de $\operatorname{Pic} \mathscr{X} / S$ dans $\mathscr{P}$ ic $(\mathscr{X} / S)$ tel que $\pi$ os soit égal à l'identité.

b) Il existe un isomorphisme de $\mathrm{B} \mathbb{G}_{\mathrm{m}} \times{ }_{S} \operatorname{Pic} \mathscr{X} / S$ dans $\mathscr{P}$ ic $(\mathscr{X} / S)$ compatible avec les projections sur $\operatorname{Pic} \mathscr{X} / S$.

c) Il existe sur le $S$-champ (non nécessairement algébrique) $\mathscr{X} \times{ }_{S} \operatorname{Pic} \mathscr{X} / S$ un faisceau inversible "universel » $\mathscr{P}$ qui représente le foncteur $\operatorname{Pic} \mathscr{X} / S$, i.e. tel que pour tout $U \in \mathrm{ob}(\mathrm{Aff} / S)$ et tout élément l de $\operatorname{Pic} \mathscr{X} / S(U)$ on ait $l=\left[\mathscr{P}_{\mid \mathscr{X} \times S_{S} U}\right]=\pi\left(\mathscr{P}_{\mid \mathscr{X} \times{ }_{S} U}\right)$.

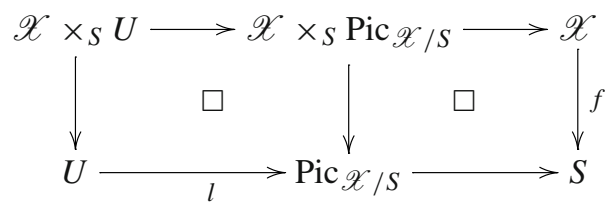

(ii) Les propositions suivantes sont équivalentes:

d) Le morphisme naturel de $P_{\mathscr{X} / S}$ dans $\operatorname{Pic} \mathscr{X} / S$ est un isomorphisme.

e) Il existe un élément de $P_{\mathscr{X} / S}\left(\operatorname{Pic}_{\mathscr{X} / S}\right)\left(c\right.$ 'est-à-dire $\operatorname{Hom}\left(\operatorname{Pic}_{\mathscr{X} / S}, P_{\mathscr{X} / S}\right)$ lorsque Pic $\mathscr{X} / S$ n'est pas représentable) qui a pour image l'identité dans $\operatorname{Pic}_{\mathscr{X} / S}\left(\operatorname{Pic}_{\mathscr{X} / S}\right)\left(\right.$ ou, ce qui revient au même, $P_{\mathscr{X} / S} \rightarrow \operatorname{Pic}_{\mathscr{X} / S}$ a une section).

(iii) Les conditions de (i) impliquent celles de (ii), et la réciproque est vraie si $\mathrm{Pic} \mathscr{X} / S$ est représentable par un schéma. Toutes ces conditions sont vérifiées si le morphisme structural $f: \mathscr{X} \rightarrow S$ a une section.

Démonstration. Les implications $b \Rightarrow a \Rightarrow e, c \Rightarrow a$ et $d \Leftrightarrow e$ sont évidentes.

Montrons que a) implique b). Soit $s$ une section de $\pi$. On vérifie facilement que le morphisme $(\mathscr{M}, l) \mapsto\left(f^{*} \mathscr{M}\right) \otimes s(l)$ de $\mathrm{B} \mathbb{G}_{\mathrm{m}} \times{ }_{S} \operatorname{Pic} \mathscr{X} / s$ dans $\mathscr{P} i c(\mathscr{X} / S)$ est un isomorphisme. (On utilise ici la platitude cohomologique en dimension zéro.) Un quasi-inverse est donné par le foncteur de $\mathscr{P} i c(\mathscr{X} / S)$ vers $\mathrm{B} \mathbb{G}_{\mathrm{m}} \times{ }_{S} \operatorname{Pic} \mathscr{X} / S$ qui à un faisceau inversible $\mathscr{M}$ associe le couple $\left(\mathscr{M} \otimes s(\pi(\mathscr{M}))^{-1}, \pi(\mathscr{M})\right)$, en identifiant $\mathrm{B} \mathbb{G}_{\mathrm{m}}$ à son image essentielle dans $\mathscr{P} i c(\mathscr{X} / S)$.

Montrons maintenant que a) implique c $)^{3}$. On se donne une section $s$ de $\pi$ et l'on va construire un faisceau inversible $\mathscr{P}$ (au sens de [37]) sur le champ $\mathscr{X} \times{ }_{S} \mathrm{Pic} \mathscr{X} / S$. Il faut donc construire pour tout $S$-schéma affine $U$ et tout objet $x$ de $\left(\mathscr{X} \times{ }_{S} \operatorname{Pic} \mathscr{X} / S\right)_{U}$ un faisceau inversible $\mathscr{P}(x)$ sur $U$, et des isomorphismes de transition entre les $\mathscr{P}(x)$. Soit $x$ un objet de $\left(\mathscr{X} \times{ }_{S} \operatorname{Pic} \mathscr{X} / S\right)_{U}$. On note $l=f_{P} \circ x$ et $t_{x}$ la section de $f_{U}$ induite

\footnotetext{
${ }^{3}$ C'est évident si Pic $\mathscr{X} / S$ est représentable par un schéma! Ce que l'on ne suppose pas ici...
} 
par $x$, comme dans le diagramme ci-dessous.

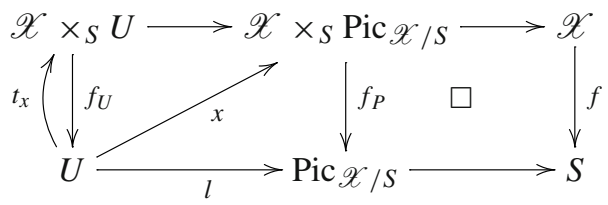

On a un faisceau inversible $s(l)$ sur $\mathscr{X} \times{ }_{s} U$. On pose $\mathscr{P}(x)=t_{x}^{*} s(l)$. Les isomorphismes de transition sont définis de manière évidente et l'on obtient ainsi un faisceau inversible $\mathscr{P}$ sur $\mathscr{X} \times{ }_{S} \operatorname{Pic} \mathscr{X} / S$ dont il ne reste plus qu'à montrer qu'il représente Pic $\mathscr{X} / S$. Soit $l$ un élément de $\operatorname{Pic} \mathscr{X}_{S S}(U)$. Il faut montrer que $l=\pi\left(\mathscr{P}_{\mid \mathscr{X} \times_{S} U}\right)$. Il est évident, vu la construction de $\mathscr{P}$, que $\mathscr{P}_{\mid \mathscr{X} \times{ }_{S} U}$ est isomorphe à $s(l)$. Comme $s$ est une section de $\pi$, on a bien le résultat attendu.

Supposons que $\operatorname{Pic} \mathscr{X} / S$ soit représentable par un schéma et montrons que e) implique c). Sous l'hypothèse e), il existe par définition du foncteur $P_{\mathscr{X} / S}$ un faisceau inversible $\mathscr{P}$ sur $\mathscr{X} \times{ }_{S} \operatorname{Pic} \mathscr{X} / S$ qui induit l'élément identité de $\mathrm{Pic} \mathscr{X} / S(\operatorname{Pic} \mathscr{X} / S)$. Il est clair que $\mathscr{P}$ est universel.

Enfin dans le cas où $f$ a une section $\sigma$, montrons que la condition b) est vérifiée. Le morphisme $f^{*}$ admet dans ce cas une rétraction $\sigma^{*}$.

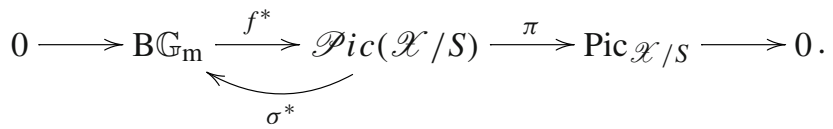

On vérifie alors facilement que le 1-morphisme $\mathscr{M} \mapsto\left(\sigma^{*} \mathscr{M}, \pi(\mathscr{M})\right)$ est un isomorphisme de $\mathscr{P}$ ic $(\mathscr{X} / S)$ dans $\mathrm{B} \mathbb{G}_{\mathrm{m}} \times{ }_{S} \operatorname{Pic} \mathscr{X} / S$.

Remarque 2.3.5 On se donne à la fois une section $\sigma$ de $f$ et un faisceau inversible universel $\mathscr{P}$ sur $\mathscr{X} \times{ }_{S} \operatorname{Pic} \mathscr{X} / S$ (on ne suppose pas Pic $\mathscr{X} / S$ représentable). Alors l'isomorphisme $\mathrm{B} \mathbb{G}_{\mathrm{m}} \times{ }_{S} \operatorname{Pic} \mathscr{X} / S \rightarrow \mathscr{P} i c(\mathscr{X} / S)$ induit par $\mathscr{P}$ est un quasi-inverse de $\left(\sigma^{*}, \pi\right)$ si et seulement si $\sigma^{*} \mathscr{P}$ est trivial. En particulier on obtient toujours un quasi-inverse en «rigidifiant» $\mathscr{P}$ le long de $\sigma$, c'est-à-dire en remplaçant $\mathscr{P}$ par $\mathscr{P} \otimes\left(f^{*} \sigma^{*} \mathscr{P}\right)^{-1}$.

Corollaire 2.3.6 Soit $\mathscr{X}$ un S-champ algébrique localement de présentation finie et cohomologiquement plat en dimension zéro. On suppose que le foncteur de Picard Pic $\mathscr{X} / S$ est représentable par un espace algébrique. Alors le champ de Picard $\mathscr{P}$ ic $(\mathscr{X} / S)$ est algébrique.

Démonstration. Pour un $S$-champ, être algébrique est une condition locale pour la topologie fppf. On peut donc supposer (2.2.4) que le morphisme de $\mathscr{X}$ dans $S$ a une section et il suffit d'appliquer la proposition précédente.

On peut donner une autre interprétation de l'équivalence entre b) et c).

Corollaire 2.3.7 Soit $\omega$ l'élément du groupe $H^{2}\left(\operatorname{Pic}_{\mathscr{X} / S}, \mathbb{G}_{\mathrm{m}}\right)$ défini par la $\mathbb{G}_{\mathrm{m}}$-gerbe $\mathscr{P}$ ic $(\mathscr{X} / S)$. Alors $\omega$ est une classe d'obstruction à l'existence d'unfaisceau inversible 
universel sur $\mathscr{X} \times{ }_{S} \operatorname{Pic} \mathscr{X}_{/ S}$ (au sens où un tel faisceau existe si et seulement si $\omega$ est nul).

\section{Représentabilité}

\subsection{Un champ algébrique au sens d'Artin}

Nous donnons dans cette partie une démonstration directe, en termes de faisceaux inversibles, du théorème d'Aoki cité en introduction.

\section{DÉFORMATIONS DE FAISCEAUX INVERSIBLES}

Commençons par une petite remarque d'algèbre que nous utiliserons abondamment par la suite et que, par commodité, nous énonçons sous la forme d'un lemme.

Lemme 3.1.1 Soient $A$ un anneau et I un idéal de carré nul de A. On note $\pi$ la projection canonique $\pi: A \rightarrow A / I$.

1) Le morphisme de groupes abéliens $\pi^{\times}: A^{\times} \rightarrow(A / I)^{\times}$induit par $\pi$ est surjectif.

2) L'application $x \mapsto 1+x$ induit un isomorphisme de groupes abéliens de I sur $\operatorname{Ker} \pi^{\times}$.

Soit $\mathscr{X}$ un champ algébrique sur un schéma $T$ et soit $\mathscr{L}$ un faisceau inversible sur $\mathscr{X}$. On considère une immersion fermée

$$
i: \mathscr{X} \longrightarrow \widetilde{\mathscr{X}}
$$

définie par un idéal quasi-cohérent $I$ de $\widetilde{\mathscr{X}}$ de carré nul.

Remarque 3.1.2 Si $\mathscr{X}$ et $\widetilde{\mathscr{X}}$ sont des champs de Deligne-Mumford, le morphisme $i$ induit une équivalence de sites entre les sites étales de $\mathscr{X}$ et de $\widetilde{\mathscr{X}}$, ce qui permet d'identifier les topos étales de $\mathscr{X}$ et de $\widetilde{\mathscr{X}}$. Il est alors évident que la catégorie des $\mathscr{O}_{\mathscr{X}}$-modules quasi-cohérents est équivalente à la catégorie des $\mathscr{O}_{\mathscr{X}}$-modules quasicohérents annulés par $I$. Lorsque l'on travaille avec des champs d'Artin (donc avec leurs sites lisses-étales) il faut être plus prudent. En effet le foncteur naturel du site lisse-étale de $\widetilde{\mathscr{X}}$ vers celui de $\mathscr{X}$ n'est même plus fidèle si bien que ces derniers ne sont a priori pas équivalents. Cependant, la descente fidèlement plate des modules quasi-cohérents ( $c f$. par exemple [34, (13.5)]) nous permet encore d'identifier la caté-

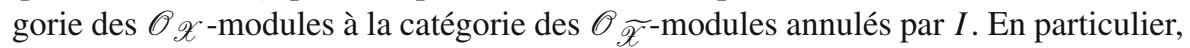
l'idéal $I$ peut être vu comme un $\mathscr{O} \mathscr{X}$-module. De plus si $\mathscr{F}$ est un faisceau abélien (non nécessairement quasi-cohérent) sur $\mathscr{X}$, alors d'après A.3.5 les groupes de cohomologie $H^{n}(\mathscr{X}, \mathscr{F})$ et $H^{n}\left(\widetilde{\mathscr{X}}, i_{*} \mathscr{F}\right)$ sont isomorphes. Ceci s'applique en particulier à $I$ (vu comme $\mathscr{O} \mathscr{X}$-module) et l'on en déduit que les groupes $H^{n}(\mathscr{X}, I)$ et $H^{n}(\widetilde{\mathscr{X}}, I)$ sont isomorphes.

On note $\operatorname{Defm}(\mathscr{L})$ la catégorie des déformations de $\mathscr{L}$ à $\widetilde{\mathscr{X}}$ définie de la manière suivante. Un objet de $\operatorname{Defm}(\mathscr{L})$ est un couple $(\widetilde{\mathscr{L}}, \lambda)$ où $\widetilde{\mathscr{L}}$ est un faisceau inversible 
sur $\widetilde{\mathscr{X}}$ et $\lambda$ est un isomorphisme $\lambda: i^{*} \widetilde{\mathscr{L}} \stackrel{\sim}{\rightarrow} \mathscr{L}$. Un morphisme de $(\widetilde{\mathscr{L}}, \lambda)$ vers $(\widetilde{\mathscr{M}}, \mu)$ est un isomorphisme $\alpha: \widetilde{\mathscr{L}} \stackrel{\sim}{\rightarrow} \widetilde{\mathscr{M}}$ tel que $\mu \circ i^{*} \alpha=\lambda$. On note $\overline{\operatorname{Defm}(\mathscr{L})}$ l'ensemble des classes d'isomorphie de $\operatorname{Defm}(\mathscr{L})$. Le théorème ci-dessous donne une description de la catégorie des déformations de $\mathscr{L}$ à $\widetilde{\mathscr{X}}$. Un petit calcul de complexe cotangent (voir [15, lemme 3.2.3]) permet de le déduire du théorème analogue démontré par Aoki pour les déformations de morphismes de champs algébriques (cf. [11, 2.1]). Il est cependant nettement plus facile à obtenir que ce dernier, comme nous pouvons le voir ci-dessous.

Théorème 3.1.3 (1) Il existe un élément $\omega \in H^{2}(\mathscr{X}, I)$ dont l'annulation équivaut à l'existence d'une déformation de $\mathscr{L}$ à $\widetilde{\mathscr{X}}$.

(2) Si $\omega=0$, alors $\overline{\operatorname{Defm}(\mathscr{L})}$ est un torseur sous $H^{1}(\mathscr{X}, I)$.

(3) Si $(\tilde{\mathscr{L}}, \lambda)$ est une déformation de $\mathscr{L}$, son groupe d'automorphismes est isomorphe à $H^{0}(\mathscr{X}, I)$.

Démonstration. Commençons par le troisième point. Par définition un automorphisme de $(\widetilde{\mathscr{L}}, \lambda)$ est un automorphisme $\varphi$ de $\widetilde{\mathscr{L}}$ qui induit l'identité de $i^{*} \widetilde{\mathscr{L}}$. Autrement dit, $\operatorname{Aut}_{\operatorname{Defm}(\mathscr{L})}(\tilde{\mathscr{L}}, \lambda)$ est le noyau du morphisme $\operatorname{Aut}(\widetilde{\mathscr{L}}) \rightarrow \operatorname{Aut}(\mathscr{L})$ induit par $i^{*}$, c'est-à-dire le noyau du morphisme

$$
H^{0}\left(\widetilde{\mathscr{X}}, \mathscr{O}_{\widetilde{\mathscr{X}}}\right)^{\times} \longrightarrow\left(\frac{H^{0}\left(\widetilde{\mathscr{X}}, \mathscr{O}_{\mathscr{X}}\right)}{H^{0}(\widetilde{\mathscr{X}}, I)}\right)^{\times}
$$

D'après le lemme 3.1.1,2), il est donc isomorphe à $H^{0}(\widetilde{\mathscr{X}}, I)$ via l'application $x \mapsto$ $1+x$.

Montrons maintenant que $\overline{\operatorname{Defm}(\mathscr{L})}$ est isomorphe à l'ensemble $\operatorname{Pic}_{[\mathscr{L}]}(\widetilde{\mathscr{X}})$ des éléments de $\operatorname{Pic}(\widetilde{\mathscr{X}})$ qui sont envoyés sur la classe $[\mathscr{L}]$ de $\mathscr{L}$ dans $\operatorname{Pic}(\mathscr{X})$. On a une application naturelle $\phi: \overline{\operatorname{Defm}(\mathscr{L})} \rightarrow \operatorname{Pic}_{[\mathscr{L}]}(\widetilde{\mathscr{X}})$ qui à une déformation $(\widetilde{\mathscr{L}}, \lambda)$ associe la classe de $\widetilde{\mathscr{L}}$ dans $\operatorname{Pic}(\widetilde{\mathscr{X}})$. Elle est clairement surjective. De plus si $(\widetilde{\mathscr{L}}, \lambda)$ et $(\widetilde{\mathscr{M}}, \mu)$ sont tels que $[\widetilde{\mathscr{L}}]=[\widetilde{\mathscr{M}}]$, il existe un isomorphisme $\alpha: \widetilde{\mathscr{L}} \rightarrow \widetilde{\mathscr{M}}$. On va montrer que l'on peut choisir $\alpha$ de telle sorte que $i^{*} \alpha$ soit égal à $\mu^{-1} \circ \lambda$. Il suffit pour cela de voir que $\operatorname{Aut}(\widetilde{\mathscr{L}}) \rightarrow \operatorname{Aut}\left(i^{*} \tilde{\mathscr{L}}\right)$ est surjectif : il n'y aura plus alors qu'à corriger $\alpha$ par un automorphisme convenablement choisi de $\widetilde{\mathscr{L}}$. Or ce morphisme de groupes s'identifie à $\operatorname{Aut}\left(\mathscr{O}_{\widetilde{X}}\right) \rightarrow \operatorname{Aut}\left(\mathscr{O}_{\mathscr{X}}\right)$, qui est surjectif en vertu de $\left.3.1 .1,1\right)$.

Donc $\overline{\operatorname{Defm}(\mathscr{L})}$ est naturellement un torseur sous le noyau du morphisme $\operatorname{Pic}(\widetilde{\mathscr{X}}) \rightarrow \operatorname{Pic}(\mathscr{X})$. Calculons ce noyau. On a une suite exacte de faisceaux quasicohérents sur $\widetilde{\mathscr{X}}$ :

$$
0 \longrightarrow I \longrightarrow \mathscr{O}_{\mathscr{X}} \longrightarrow i_{*} \mathscr{O}_{\mathscr{X}} \longrightarrow 0
$$

Elle induit via l'application exponentielle ( $c f .3 .1 .1)$ une suite exacte de faisceaux abéliens sur $\widetilde{\mathscr{X}}$ :

$$
0 \longrightarrow I \longrightarrow \mathscr{O}_{\widetilde{X}}^{\times} \longrightarrow i_{*} \mathscr{O}_{\mathscr{X}}^{\times} \longrightarrow 0 .
$$


La suite exacte longue de cohomologie associée nous donne:

$$
H^{1}(\widetilde{\mathscr{X}}, I) \longrightarrow H^{1}\left(\widetilde{\mathscr{X}}, \mathscr{O}_{\widetilde{\mathscr{X}}}^{\times}\right) \longrightarrow H^{1}\left(\widetilde{\mathscr{X}}, i_{*} \mathscr{O}_{\mathscr{X}}^{\times}\right) \longrightarrow H^{2}(\widetilde{\mathscr{X}}, I)
$$

Or d'après 2.1.2, le groupe $H^{1}\left(\widetilde{\mathscr{X}}, \mathscr{O}_{\widetilde{\mathscr{X}}}^{\times}\right)$est isomorphe à $\operatorname{Pic}(\widetilde{\mathscr{X}})$. De plus le lemme A.3.5 fourni en annexe montre que $H^{1}\left(\widetilde{\mathscr{X}}, i_{*} \mathscr{O}_{\mathscr{X}}^{\times}\right) \simeq H^{1}\left(\mathscr{X}, \mathscr{O}_{\mathscr{X}}^{\times}\right) \simeq \operatorname{Pic}(\mathscr{X})$. Montrons enfin que la première flèche de la suite exacte longue ci-dessus est injective. Il suffit pour cela de voir que le morphisme $H^{0}\left(\widetilde{\mathscr{X}}, \mathscr{O}_{\widetilde{\mathscr{X}}}^{\times}\right) \rightarrow H^{0}\left(\widetilde{\mathscr{X}}, i_{*} \mathscr{O}_{\mathscr{X}}^{\times}\right)$est surjectif, ce qui résulte encore du lemme 3.1.1. On obtient donc la suite exacte

$$
0 \longrightarrow H^{1}(\widetilde{\mathscr{X}}, I) \longrightarrow \operatorname{Pic}(\widetilde{\mathscr{X}}) \longrightarrow \operatorname{Pic}(\mathscr{X}) \longrightarrow H^{2}(\widetilde{\mathscr{X}}, I)
$$

ce qui achève notre démonstration.

Remarque 3.1.4 Nous avons montré au passage que le noyau de $\operatorname{Pic}(\widetilde{\mathscr{X}}) \rightarrow \operatorname{Pic}(\mathscr{X})$ s'identifie à $H^{1}(\widetilde{\mathscr{X}}, I)$.

Nous pouvons maintenant utiliser le théorème [13, (5.3)] d'Artin pour (re)démontrer le théorème 1.1 .

Nouvelle démonstration du théorème 1.1 On note $f$ le morphisme de $\mathscr{X}$ dans $S$ et $\mathscr{P}$ le champ $\mathscr{P}$ ic $(\mathscr{X} / S)$. Si $A$ est un anneau sur $S$, on note $\mathscr{P}(A)$ la catégorie fibre de $\mathscr{P}$ au-dessus de Spec $A$. On peut supposer que $S$ est le spectre d'un anneau $R$. On peut même supposer que $R$ est de type fini sur $\mathbb{Z}$ grâce à la proposition (4.18) (ii) de [34]. On vérifie une à une les conditions de [13, (5.3)].

\section{- Présentation finie}

Il faut montrer que si $A$ est une limite inductive d'anneaux noethériens $\lim _{\longrightarrow} A_{i}$, alors le foncteur

$$
\lim _{\longrightarrow} \mathscr{P}\left(A_{i}\right) \longrightarrow \mathscr{P}(A)
$$

est une équivalence de catégories. Ceci résulte immédiatement de la proposition (4.18) de [34] compte tenu du fait que $\mathscr{P}(A)$ s'identifie à $\operatorname{Hom}_{A}\left(\mathscr{X}_{A}, \mathrm{~B} \mathbb{G}_{\mathrm{m}}\right)$ et que $\mathrm{B} \mathbb{G}_{\mathrm{m}}$ est de présentation finie. (Ici un indice comme $\mathscr{X}_{A}$ désigne le changement de base $\left.\mathscr{X} \times_{S} \operatorname{Spec} A\right)$.

- Première condition de Schlessinger (appelée (S1') dans [13, p. 168])

On se donne un carré cartésien d'anneaux noethériens

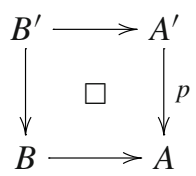


où $A^{\prime}$ est une extension infinitésimale de $A$. Il faut montrer que pour tout objet $a$ de $\mathscr{P}(A)$ le foncteur

$$
\mathscr{P}_{a}\left(B^{\prime}\right) \longrightarrow \mathscr{P}_{a}\left(A^{\prime}\right) \times \mathscr{P}_{a}(B)
$$

est une équivalence de catégories, où pour tout anneau $R^{\prime}$ sur $A, \mathscr{P}_{a}\left(R^{\prime}\right)$ désigne la catégorie des objets de $\mathscr{P}\left(R^{\prime}\right)$ au-dessus de $a$ avec les morphismes au-dessus de l'identité de $a$. C'est une conséquence immédiate du théorème suivant.

Théorème 3.1.5 Si $\mathscr{X}$ est un champ algébrique plat sur $S$, le foncteur naturel

$$
F(\mathscr{X}): \operatorname{Inv}\left(\mathscr{X}_{B^{\prime}}\right) \longrightarrow \operatorname{Inv}\left(\mathscr{X}_{A^{\prime}}\right) \times \operatorname{Inv}\left(\mathscr{X}_{A}\right) \operatorname{Inv}\left(\mathscr{X}_{B}\right)
$$

est une équivalence de catégories (où Inv ( $\mathscr{X}$ ) est la catégorie des faisceaux inversibles sur $\mathscr{X})$.

Démonstration. Commençons par l'observation suivante. Soient $\mathscr{U}$ un $S$-champ algébrique, $U^{1}$ un $S$-espace algébrique, et soit $U^{1} \rightarrow \mathscr{U}$ un épimorphisme. On note $U^{2}=U^{1} \times \mathscr{U} U^{1}$ et $U^{3}=U^{1} \times \mathscr{U} U^{1} \times \mathscr{U} U^{1}$. Alors la catégorie $\operatorname{Inv}(\mathscr{U})$ est équivalente à la catégorie $\operatorname{Desc}\left(U^{1}, \mathscr{U}\right)$ décrite dans 2.1.1. De plus la construction de $\operatorname{Desc}\left(U^{1}, \mathscr{U}\right)$ commute aux produits fibrés de champs, de sorte que si les foncteurs naturels $F\left(U^{i}\right)$ pour $i=1,2,3$, sont des équivalences de catégories, il en est de même du foncteur $F(\mathscr{U})$.

Revenons à la démonstration proprement dite du théorème 3.1.5.

Premier cas : $\mathscr{X}$ est un $S$-schéma affine Spec $R^{\prime}$.

Alors l'assertion résulte du théorème [21,2.2] et du lemme suivant.

Lemme 3.1.6 Si $R^{\prime}$ est une R-algèbre plate, alors le carré obtenu par changement de base

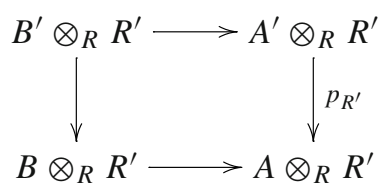

est encore cartésien, et $p_{R^{\prime}}$ est surjectif.

$2^{e ̀ m e}$ cas : $\mathscr{X}$ est un $S$-schéma qui est union disjointe de schémas affines.

On a $\mathscr{X}=\coprod_{i} X_{i}$, où chaque $X_{i}$ est affine. Alors Inv $(\mathscr{X})=\prod_{i} \operatorname{Inv}\left(X_{i}\right)$ universellement, et l'assertion est vraie pour chacun des $S$-schémas $X_{i}$, donc il suffit de montrer que les produits fibrés de catégories commutent aux produits arbitraires, ce qui est purement formel et évident.

$3^{\text {ème }}$ cas : $\mathscr{X}$ est un schéma séparé.

Soit $X$ l'union disjointe d'une famille d'ouverts affines recouvrant $\mathscr{X}$. Alors $X, X \times \mathscr{X}$ $X$, et $X \times \mathscr{X} X \times \mathscr{X} X$ sont unions disjointes de schémas affines, donc d'après la remarque qui amorçait notre démonstration, l'assertion résulte du second cas. 
$4^{e ̀ m e}$ cas : $\mathscr{X}$ est un $S$-schéma quelconque.

De même soit $X$ la somme des ouverts d'un recouvrement affine de $\mathscr{X}$. Alors les schémas $X, X \times \mathscr{X} X$ et $X \times \mathscr{X} X \times \mathscr{X} X$ sont séparés, donc il suffit d'appliquer le troisième cas.

$5^{\text {ème }}$ cas : $\mathscr{X}$ est un $S$-espace algébrique.

On choisit un morphisme étale surjectif $X \rightarrow \mathscr{X}$ où $X$ est un schéma. Alors $X \times \mathscr{X} X$ et $X \times \mathscr{X} X \times \mathscr{X} X$ sont encore des schémas, de sorte que l'assertion résulte du cas précédent.

$6^{\text {ème }}$ cas : $\mathscr{X}$ est un $S$-champ algébrique.

On choisit une présentation $P: X \rightarrow \mathscr{X}$ par un espace algébrique. Alors $X \times \mathscr{X} X$ et $X \times \mathscr{X} X \times \mathscr{X} X$ sont encore des espaces algébriques, donc le résultat se déduit du cinquième cas.

- Seconde condition de Schlessinger (appelée (S2) dans [13, p. 168])

Soient $A$ une $R$-algèbre noethérienne et $M$ un $A$-module de type fini. On peut supposer, et l'on suppose, $A=R$. On note $A[M]$ l'extension infinitésimale de $A$ par $M$. Si $a$ est un objet de $\mathscr{P}(A)$, on note

$$
D_{a}(M)=\mathscr{P}_{a}(A[M]) / \sim
$$

Il faut vérifier que $D_{a}(M)$ est un $A$-module de type fini. Or $D_{a}(M)$ n'est autre que l'ensemble appelé $\overline{\operatorname{Defm}(a)}$ ci-dessus, et l'on a vu que cet ensemble est naturellement un torseur sous $H^{1}(\widetilde{\mathscr{X}}, I)$, où $\widetilde{\mathscr{X}}$ désigne le produit fibré $\mathscr{X} \times{ }_{S} \operatorname{Spec} A[M]$. Il suffit de vérifier que $H^{1}(\widetilde{\mathscr{X}}, I)$ est un $A$-module de type fini. On vérifie facilement que l'idéal $I$ qui définit l'extension infinitésimale $\mathscr{X} \hookrightarrow \widetilde{\mathscr{X}}$ s'identifie à $i_{*} f^{*} M$. D'après A.3.5 le module $H^{1}(\widetilde{\mathscr{X}}, I)$ est donc isomorphe à $H^{1}\left(\mathscr{X}, f^{*} M\right)$. L'assertion résulte maintenant de la finitude de la cohomologie des faisceaux cohérents sur un champ algébrique propre et localement noethérien [41, théorème (1.2)], ou [20].

- Fin de la condition (1) du théorème [13, (5.3)]

Il faut vérifier que si $a$ est un objet de $\mathscr{P}(A)$ et si $M$ est un $A$-module de type fini, alors l'ensemble $\operatorname{Aut}_{a}(M)$ des automorphismes infinitésimaux, défini par

$$
\operatorname{Aut}_{a}(M):=\operatorname{Ker}\left(\operatorname{Aut}_{a}(A[M]) \rightarrow \operatorname{Aut}_{a}(A)\right)
$$

est un $A$-module de type fini. (Dans le membre de droite, Aut ${ }_{a}$ désigne le foncteur des automorphismes de $a$. $\operatorname{Aut}_{a}(A[M])$ est donc l'ensemble des automorphismes de la déformation triviale $\tilde{a}$ de $a$, image de $a$ par le morphisme $\mathscr{P}(A) \rightarrow \mathscr{P}(A[M])$.) D'après le troisième point du théorème 3.1 .3 ci-dessus, $\operatorname{Aut}_{a}(M)$ est naturellement isomorphe à $H^{0}\left(\mathscr{X}, f^{*} M\right)$, qui est bien de type fini d'après le théorème de finitude de Faltings-Olsson [41, (1.2)].

\section{- Commutation aux limites projectives}

Il faut vérifier que si $A$ est un anneau noethérien local complet d'idéal maximal m, alors le foncteur naturel

$$
\mathscr{P}(A) \longrightarrow \lim _{\longleftarrow} \mathscr{P}\left(A / \mathfrak{m}^{n+1}\right)
$$


est une équivalence de catégories. C'est une conséquence immédiate du théorème

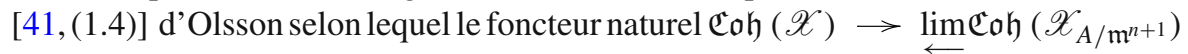
est une équivalence.

- Conditions sur la théorie des déformations

Il faut vérifier que les modules $\operatorname{Aut}_{a}(M), D_{a}(M)$ et $\mathscr{O}_{a}(M)$ (où $\mathscr{O}_{a}(M)$ doit être un module dans lequel vit une classe d'obstruction à l'existence d'une déformation de l'élément $a$ à $A[M], c f$. [13, (2.6)]) vérifient les conditions (4.1) de [13], c'est-à-dire :

(i) qu'ils commutent à la localisation étale, i.e. si $A \rightarrow B$ est étale alors $\operatorname{Aut}_{b}\left(M \otimes_{A} B\right)$ (où $b$ est l'image de $a$ ) est isomorphe à $\operatorname{Aut}_{a}(M) \otimes_{A} B$, et idem pour les autres;

(ii) qu'ils sont compatibles aux limites projectives, i.e. si m est un idéal maximal de $A$ et si $\hat{A}$ est le complété de $A$ relativement à m, alors le morphisme naturel

$$
\operatorname{Aut}_{a}(M) \otimes_{A} \hat{A} \longrightarrow \lim _{\longleftarrow} \operatorname{Aut}_{a_{n}}\left(M / \mathfrak{m}^{n} M\right)
$$

est un isomorphisme, etc...

(iii) (constructibilité) Il existe un ouvert dense de points de type fini $p \in \operatorname{Spec} A$ tels que

$$
\operatorname{Aut}_{a}(M) \otimes_{A} \kappa(p) \longrightarrow \operatorname{Aut}_{a_{p}}\left(M \otimes_{A} \kappa(p)\right)
$$

soit un isomorphisme, etc...

Or d'après ce qui précède, ces modules s'identifient respectivement à $H^{0}\left(\mathscr{X}, f^{*} M\right)$, $H^{1}\left(\mathscr{X}, f^{*} M\right)$ et $H^{2}\left(\mathscr{X}, f^{*} M\right)$. La condition (i) résulte de la proposition A.3.3. La condition (ii) est une conséquence de [40] thm. 8.1.

Enfin pour la condition (iii), nous devons montrer que si $A_{0}$ est une $R$-algèbre intègre de type fini, il existe un ouvert non vide $U$ de Spec $A_{0}$ tel que pour tout point fermé $s$ dans $U$ le $\kappa(s)$-espace vectoriel $D\left(k, M \otimes_{A_{0}} \kappa(s), \xi_{0_{s}}\right)$ soit isomorphe à $D\left(A_{0}, M, \xi_{0}\right) \otimes_{A_{0}} \kappa(s)$. En d'autres termes on demande que pour un tel point $s$, le morphisme canonique

$$
H^{q}\left(\mathscr{X}_{A_{0}}, f_{A_{0}}^{*} M\right) \otimes_{A_{0}} \kappa(s) \rightarrow H^{q}\left(\mathscr{X}_{s}, f_{s}^{*}\left(M \otimes_{A_{0}} \kappa(s)\right)\right)
$$

soit un isomorphisme ( $q=0,1$ ou 2). Montrons d'abord qu'il existe un ouvert non vide de Spec $A_{0}$ sur lequel $M$ est libre de rang fini. On note $K$ le corps des fractions de $A_{0}$. Comme $M$ est de type fini, $M_{K}$ est un $K$-espace vectoriel de dimension finie. Soient $\left(x_{1}, \ldots, x_{n}\right)$ des éléments de $M$ dont les images dans $M_{K}$ engendrent $M_{K}$. Soit $\left(y_{1}, \ldots, y_{n}\right)$ une famille génératrice de $M$. Il existe $a_{0} \in A_{0}$ non nul tel que pour tout $j$ on puisse écrire $y_{j}$ comme combinaison linéaire des $\frac{x_{i}}{a_{0}}$ dans $M_{K}$. Donc quitte à localiser par $a_{0}$ on peut supposer que la famille $\left(x_{1}, \ldots, x_{n}\right)$ engendre $M$. C'est alors aussitôt une base de $M$. En effet, on a un morphisme surjectif $\varphi: A^{n} \rightarrow M$ qui induit un isomorphisme $\varphi_{K}: A_{K}^{n} \rightarrow M_{K}$, et le morphisme de localisation $A^{n} \rightarrow K^{n}$ est injectif puisque $A$ est intègre, donc $\varphi$ est aussi injectif. Maintenant, $M$ est libre de rang fini sur Spec $A_{0}$, donc $f_{A_{0}}^{*} M \simeq\left(\mathscr{O}_{\mathscr{X} \times{ }_{S} \operatorname{Spec} A_{0}}\right)^{n}$ est cohérent et plat sur Spec $A_{0}$ 
(car $f$ est plat). Donc le résultat de Mumford ([38] paragraphe 5) généralisé par Aoki aux champs algébriques [11, théorème A.1], s'applique et il existe un ouvert non vide $U$ de Spec $A_{0}$ tel que, pour tout point $s$ de $U$, le morphisme (1) ci-dessus soit un isomorphisme.

- Quasi-séparation du morphisme diagonal

Soient $A$ une $R$-algèbre de type fini et $\mathscr{L}$ un faisceau inversible sur $\mathscr{X}_{A}$. Soit $\varphi$ un automorphisme de $\mathscr{L}$ qui induit l'identité dans $\mathscr{P}(k)$ pour un ensemble dense de points $A \rightarrow k$. Il faut montrer que $\varphi$ est l'identité sur un ouvert non vide de Spec A. Remarquons tout de suite qu'en fait, en lisant la preuve de [13] (5.3) (haut de la page 184, voir aussi [12, p. 59, Sect. 2] et regarder la condition [3'](b) du théorème 5.3 dans [12], p. 48), on voit qu'on peut supposer $A$ intègre. Soit $X \rightarrow \mathscr{X}$ une présentation de $\mathscr{X}$, avec $X$ affine d'anneau $B$. On note encore $\varphi$ l'élément de $\Gamma\left(\mathscr{X}_{A}, \mathscr{O}_{\mathscr{X}_{A}}\right)^{\times}$correspondant à l'automorphisme $\varphi$. Il suffit clairement de montrer que $\varphi_{\left.\right|_{X}}$ vaut 1 . Notons $b$ l'élément $\varphi_{\left.\right|_{X}}-1$ de $B$, et $H$ l'ensemble des points $p$ de Spec $A$ tels que $b_{p}=0$. Par hypothèse $H$ est dense dans Spec $A$. Par ailleurs, d'après [29] (9.4.6), $H$ est constructible. Donc le point générique $\xi$ de Spec $A$ est dans $H$ et $b_{\xi}=0$. Comme $B$ est plat sur $A$, ceci prouve que $b$ est nul.

\subsection{Quasi-séparation et séparation}

Nous montrons maintenant que, sous des hypothèses légèrement plus fortes qu'en 1.1 (on suppose de plus $\mathscr{X}$ cohomologiquement plat en dimension zéro sur $S$ ), le champ de Picard est algébrique au sens de [34], c'est-à-dire en un sens un peu plus fort que celui d'Artin. La différence porte uniquement sur les conditions de finitude imposées au morphisme diagonal : les champs algébriques de [34] sont supposés quasi-séparés, autrement dit leur diagonale est quasi-compacte (et séparée). La question de la quasi-séparation étant parfois délicate, nous proposons ci-dessous un critère général de quasi-séparation, inspiré des techniques employées par Artin dans [12]. La fin de cette section est consacrée à un critère de séparation pour le foncteur de Picard (cf. 3.2.5).

Proposition 3.2.1 Soient $S$ un schéma localement noethérien et $\mathscr{X}$ un $S$-champ algébrique (au sens d'Artin) localement de présentation finie. On suppose que les deux conditions suivantes sont remplies.

(i) Pour tout $U \in \mathrm{ob}(\mathrm{Aff} / S)$ et tout $x \in \mathrm{ob} \mathscr{X}_{U}$, le morphisme $\underline{\operatorname{Aut}}(x) \rightarrow U$ est quasi-compact et séparé.

(ii) Soit $U \in \mathrm{ob}(\mathrm{Aff} / S)$ intègre, et soient $x, y \in \mathrm{ob} \mathscr{X}_{U}$. On suppose qu'il existe un ensemble dense de points $t$ de $U$, tels qu'il existe une extension $L(t)$ de $\kappa(t)$ telle que $x_{L(t)} \simeq y_{L(t)}$. Alors $x$ et $y$ sont isomorphes sur un ouvert dense de $U$.

Alors $\mathscr{X}$ est quasi-séparé (donc est algébrique au sens de [34]).

Démonstration. Soit $U \rightarrow \mathscr{X} \times_{S} \mathscr{X}$ une présentation de $\mathscr{X} \times_{S} \mathscr{X}$, où $U$ est un schéma localement noethérien. Alors par descente fidèlement plate, pour montrer que $\Delta: \mathscr{X} \rightarrow \mathscr{X} \times_{S} \mathscr{X}$ est quasi-compact, il suffit de montrer que le morphisme 
obtenu par changement de base

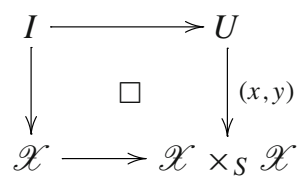

est quasi-compact. Comme c'est une question locale sur $U$, on peut supposer $U$ affine.

Notons $G$ le $U$-schéma en groupes $\underline{\operatorname{Aut}}(y)$. L'action à gauche naturelle de $G$ sur $I$ fait clairement de $I \rightarrow U$ un pseudo-torseur ${ }^{4}$ sous $G$. Par récurrence noethérienne sur les fermés de $U$, on peut supposer que pour tout fermé strict $F$ de $U, I \times_{U} F$ est quasi-compact. Il suffit maintenant de trouver un ouvert non vide $V$ de $U$ tel que $I \times{ }_{U} V$ soit quasi-compact. En effet, si $V$ est un tel ouvert, alors en notant $F=U \backslash V$, l'espace algébrique $I^{\prime}=\left(I \times_{U} V\right) \coprod\left(I \times_{U} F\right)$ est quasi-compact, et le morphisme $I^{\prime} \rightarrow I$ obtenu par changement de base à partir de $V \amalg F \rightarrow U$ est surjectif, donc $I$ est quasi-compact.

Notons $f$ le morphisme $I \rightarrow U$ et $Z=\overline{f(I)}$. Soit $W$ un ouvert affine intègre

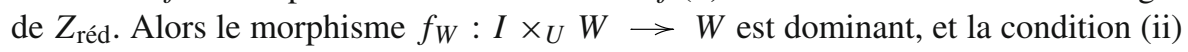
signifie précisément que dans ce cas, quitte à choisir $W$ plus petit, $f_{W}$ a une section. Ceci prouve que $I \times_{U} W$ est un torseur trivial sous $G$, donc est quasi-compact. Ceci étant, on peut écrire $W=Z_{\text {réd }} \cap V=Z_{\text {réd }} \times_{U} V$ où $V$ est un ouvert (non vide) de $U$. On vérifie sans peine que le morphisme induit $I \times_{U} W \rightarrow I \times{ }_{U} V$ est surjectif (c'est même un homéomorphisme), ce qui montre que $I \times_{U} V$ est quasi-compact, et achève la démonstration.

Pour montrer que $\Delta$ est séparé, il faut montrer que pour tout diagramme comme ci-dessus, le morphisme $I \rightarrow U$ est séparé. En utilisant le critère valuatif [34] (A.3), on peut supposer que $U$ est le spectre d'un anneau de valuation discrète et il faut alors montrer que deux sections quelconques de $I \rightarrow U$ sont égales si et seulement si elles sont égales sur la fibre générique. Or si $I \rightarrow U$ a une section, $I$ est isomorphe au schéma $\underline{\operatorname{Aut}}(x)$ pour un certain objet $x$ de $\mathscr{X}_{U}$. Il est donc séparé par hypothèse.

Nous sommes maintenant en mesure de montrer que le champ de Picard est quasiséparé ( $c f .1 .2)$. Ceci résulte immédiatement du critère ci-dessus et des lemmes 3.2.2 et 3.2 .3 ci-dessous.

Lemme 3.2.2 Soient $A$ un anneau intègre, $T=\operatorname{Spec} A$, et $f: \mathscr{X} \rightarrow T$ un $T$-champ algébrique propre, plat, de présentation finie, et cohomologiquement plat en dimension zéro. Soit $\mathscr{L}$ un faisceau inversible sur $\mathscr{X}$.

(i) Si A est un anneau local (en particulier si A est un anneau de valuation discrète), de corps résiduel $k$ et de corps des fractions $K$, alors $\mathscr{L}$ est trivial si et seulement si $\mathscr{L}_{k}$ et $\mathscr{L}_{K}$ le sont.

(ii) S'il existe un ensemble dense $\mathscr{S}$ de points de $T$ tel que pour tout $t \in \mathscr{S}, \mathscr{L}_{t}$ soit trivial, alors il existe un ouvert non vide $U$ de $T$ tel que $\mathscr{L}_{U}$ soit trivial.

\footnotetext{
${ }^{4}$ Autrement dit la flèche $I \longrightarrow U$ est invariante sous $G$, et le morphisme naturel $G \times I \rightarrow I \times U I$ est un isomorphisme.
} 
Démonstration. D'après l'annexe A de [11] les corollaires 1 et 2 du paragraphe 5 de [38] sont encore valables. Ils nous apprennent que la fonction

$$
t \longmapsto d(t):=\operatorname{dim}_{\kappa(t)} H^{0}\left(\mathscr{X}_{t}, \mathscr{L}_{t}\right)
$$

est semi-continue supérieurement, et que si elle est constante sur $T$, alors le morphisme naturel

$$
\left(f_{*} \mathscr{L}\right) \otimes_{\mathscr{O}_{T}} \kappa(t) \longrightarrow H^{0}\left(\mathscr{X}_{t}, \mathscr{L}_{t}\right)
$$

est un isomorphisme pour tout $t \in T$. De plus en un point où $\mathscr{L}_{t}$ est trivial, on a $H^{0}\left(\mathscr{X}_{t}, \mathscr{L}_{t}\right)=H^{0}\left(\mathscr{X}_{t}, \mathscr{O}_{\mathscr{X}_{t}}\right) \simeq \kappa(t)$ par platitude cohomologique, donc $d(t)=1$.

(i) Ici comme $\mathscr{L}_{K}$ et $\mathscr{L}_{k}$ sont triviaux, la fonction $d$ vaut 1 au point générique et au point fermé, donc elle est constante égale à 1 sur $T$. D'après ce qui précède, on a en particulier un isomorphisme $H^{0}\left(T, f_{*} \mathscr{L}\right) \otimes_{A} k=H^{0}(\mathscr{X}, \mathscr{L}) \otimes_{A} k \rightarrow H^{0}\left(\mathscr{X}_{k}, \mathscr{L}_{k}\right)$. Le faisceau $\mathscr{L}_{k}$ étant trivial, il a une section globale partout non nulle sur $\mathscr{X}_{k}$. D'après l'isomorphisme précédent, cette section provient d'une section globale $s$ de $\mathscr{L}$ (comme $k$ est un quotient de $A$, les éléments de $H^{0}(\mathscr{X}, \mathscr{L}) \otimes_{A} k$ peuvent tous s'écrire sous la forme $s \otimes 1$ ). Alors $s$ est non nulle en tout point $x$ de $\mathscr{X}$ d'image Spec $k$ dans $T$. Or l'ensemble $C$ des points de $\mathscr{X}$ où $s$ est nulle est un fermé de $|\mathscr{X}|$ (voir par exemple $[15,2.1 .2 .2])$. Comme $f$ est propre, l'image de $C$ est un fermé de $T$, qui de plus ne contient pas le point fermé. Nécessairement $C$ est vide, donc $s$ est partout non nulle et $\mathscr{L}$ est trivial $[15,2.1 .2]$.

(ii) La fonction $d$ étant semi-continue supérieurement, il existe un ouvert non vide sur lequel elle est constante et quitte à remplacer $T$ par cet ouvert, on peut supposer qu'elle est constante. Dès lors pour tout $t \in T$ le morphisme naturel

$$
H^{0}(\mathscr{X}, \mathscr{L}) \otimes_{A} \kappa(t) \longrightarrow H^{0}\left(\mathscr{X}_{t}, \mathscr{L}_{t}\right)
$$

est un isomorphisme. Soit $t \in \mathscr{S}$. Alors $\mathscr{L}_{t}$ est trivial donc il a une section globale $s_{t} \in H^{0}\left(\mathscr{X}_{t}, \mathscr{L}_{t}\right)$ partout non nulle sur $\mathscr{X}_{t}$. D'après l'isomorphisme précédent, elle provient d'un élément du groupe $H^{0}(\mathscr{X}, \mathscr{L}) \otimes_{A} \kappa(t)$ que l'on peut écrire sous la forme $s \otimes\left(\frac{1}{f}\right)$ où $f \in A$ n'est pas dans l'idéal premier correspondant à $t$. Quitte à remplacer $T$ par $D(f)$, on peut supposer $f=1$ et on a donc trouvé une section $s \in H^{0}(\mathscr{X}, \mathscr{L})$ dont la réduction $s_{t}$ à $\mathscr{X}_{t}$ est partout non nulle.

Maintenant l'ensemble $C$ des points de $\mathscr{X}$ où $s$ est nulle est un fermé de $|\mathscr{X}|$, et son image est un fermé $F$ de $T$, qui ne contient pas $t$. Son complémentaire $U=T \backslash F$ est alors un ouvert non vide de $T$, et la section $s_{U}$ induite par $s$ sur $\mathscr{X} \times_{T} U$ est partout non nulle sur $\mathscr{X} \times_{T} U$, de sorte que $\mathscr{L}_{U}$ est trivial.

Lemme 3.2.3 Soit $\mathscr{X}$ un champ algébrique quasi-compact sur un corps $k$ et soit $\mathscr{L}$ un faisceau inversible sur $\mathscr{X}$. On suppose que $H^{0}\left(\mathscr{X}, \mathscr{O}_{\mathscr{X}}\right)=k$ (par exemple $\mathscr{X}$ cohomologiquement plat en dimension zéro) et qu'il existe une extension $k^{\prime}$ de $k$ telle que $\mathscr{L}_{k^{\prime}}$ soit trivial. Alors $\mathscr{L}$ est trivial.

Démonstration. D'après A.3.3, le groupe $H^{0}(\mathscr{X}, \mathscr{L}) \otimes_{k} k^{\prime}$ est isomorphe à $H^{0}\left(\mathscr{X}_{k^{\prime}}, \mathscr{L}_{k^{\prime}}\right)$ (lui-même isomorphe à $\left.k^{\prime}\right)$. On en déduit immédiatement que $\mathscr{L}$ est 
engendré par ses sections globales ${ }^{5}$. Il en est de même pour $\mathscr{L}^{-1}$. Soit $x$ un point de $|\mathscr{X}|$. Il existe des sections globales $s \in H^{0}(\mathscr{X}, \mathscr{L})$ et $s^{\prime} \in H^{0}\left(\mathscr{X}, \mathscr{L}^{-1}\right)$ non nulles en $x$. Alors $s \otimes s^{\prime} \in H^{0}(\mathscr{X}, \mathscr{O} \mathscr{X})$ est non nulle en $x$. Comme $H^{0}(\mathscr{X}, \mathscr{O} \mathscr{X})=k$, on en déduit que $s \otimes s^{\prime}$ est partout non nulle, donc $s$ aussi, et $\mathscr{L}$ est trivial.

Corollaire 3.2.4 Soient $S$ un schéma localement noethérien, et $\mathscr{X}$ un champ algébrique propre, plat et cohomologiquement plat sur $S$, alors le foncteur de Picard Pic $\mathscr{X} / S$ est un espace algébrique localement séparé.

Démonstration. Il faut montrer que le morphisme diagonal $\Delta$ de Pic $\mathscr{X} / S$ est une immersion quasi-compacte. Nous laissons au lecteur le soin de vérifier que la quasicompacité résulte de 1.2. Pour montrer que $\Delta$ est une immersion, on peut appliquer le critère valuatif d'Artin pour les immersion quasi-compactes, énoncé et démontré en détail dans la thèse de l'auteur, $c f$. [15] 2.1.1.4.

Proposition 3.2.5 Soient $S$ un schéma et $\mathscr{X}$ un $S$-champ algébrique propre, plat et de présentation finie sur $S$. On suppose que les fibres de $\mathscr{X}$ sont géométriquement normales et géométriquement intègres. Alors le foncteur de Picard Pic $\mathscr{X} / S$ est un espace algébrique séparé sur $S$.

Démonstration. D'après la remarque 2.2.5, $\mathscr{X}$ est cohomologiquement plat, donc on sait déjà que Pic $\mathscr{X} / S$ est un espace algébrique. On peut clairement supposer que $\mathscr{X}$ a une section et que $S$ est noethérien. Alors d'après le théorème 2.2.6, $\operatorname{Pic} \mathscr{X} / S$ est donné par $\operatorname{Pic} \mathscr{X}_{/ S}(T)=\operatorname{Pic}\left(\mathscr{X}_{T}\right) / \operatorname{Pic}(T)$. En utilisant le critère valuatif [34] (A.3), on peut supposer que $S$ est le spectre d'un anneau de valuation discrète $\Lambda$ (on notera $K$ son corps des fractions et $U=\operatorname{Spec} K$ ), et l'on est ramené à montrer qu'un faisceau inversible $\mathscr{L}$ sur $\mathscr{X}$ qui est trivial sur la fibre générique $\mathscr{X}_{U}$ est trivial. Vu que le $K$-espace vectoriel $\Gamma\left(\mathscr{X}_{U}, \mathscr{L}_{\mathscr{X}_{U}}\right)$ est isomorphe à $\Gamma(\mathscr{X}, \mathscr{L}) \otimes_{\Lambda} K($ A.3.3), il existe une section globale $l \in \Gamma(\mathscr{X}, \mathscr{L})$ qui engendre $\mathscr{L}$ en tout point de $\mathscr{X}_{U}$. Notons $\eta$ le point générique de la fibre spéciale, et $t$ une uniformisante de $\Lambda$.

Commençons par quelques considérations sur les présentations de $\mathscr{X}$. Soit $X=$ Spec $A$ un voisinage lisse affine de $\eta$ tel que la fibre spéciale de $X$ soit connexe (donc intègre, puisqu'elle est aussi normale). L'idéal de $A$ correspondant au point générique de la fibre spéciale de $X$ est $t A$. On notera $A_{(t)}$ l'anneau local en ce point. Vu que $A$ est plat sur $\Lambda$, il s'injecte dans $A \otimes_{\Lambda} K$. On en déduit d'une part qu'il est réduit, et d'autre part que $t$ est non diviseur de zéro dans $A$. Il en résulte que $A$ est intègre. En particulier $A_{(t)}$ est un anneau de valuation discrète.

- Montrons que l'on peut choisir la section l de telle sorte qu'elle engendre $\mathscr{L}$ en $\eta$. Si $X=\operatorname{Spec} A$ est un voisinage de $\eta$ comme ci-dessus, on note $n$ la valuation de $l$ dans $A_{(t)}(n$ ne dépend pas du choix de $X)$. Il suffit de montrer qu'il existe $l^{\prime} \in \Gamma(\mathscr{X}, \mathscr{L})$ tel que $l=t^{n} l^{\prime}$. Soit $\left(U_{i}\right)_{i \in I}$ un recouvrement lisse de $\mathscr{X}$ par des schémas affines intègres, dont la fibre spéciale est intègre ou vide, et qui trivialisent $\mathscr{L}$. On note $A_{i}$ l'anneau de $U_{i}$, et $l_{i}$ l'élément de $A_{i}$ induit par $l$ (bien défini à un inversible près).

\footnotetext{
5 On dit qu'un faisceau inversible $\mathscr{L}$ sur $\mathscr{X}$ est engendré par ses sections globales si pour point $x$ de $|\mathscr{X}|$, $\mathscr{L}$ admet une section globale non nulle en $x$.
} 
Que la fibre spéciale de $U_{i}$ soit intègre ou bien vide, on vérifie facilement qu'il existe un unique $l_{i}^{\prime} \in A_{i}$ tel que $l_{i}=t^{n} l_{i}^{\prime}$. Par unicité les $l_{i}^{\prime}$ se recollent, ce qui permet de conclure.

- Montrons que $\mathscr{L}$ est trivial.

On note $\mathscr{V}$ l'ouvert de $\mathscr{X}$ où la section $l$ est non nulle, et $i$ l'immersion ouverte de $\mathscr{V}$ dans $\mathscr{X}$. Alors $\mathscr{L}_{\left.\right|_{V}}$ est trivial. Nous allons montrer que les morphismes canoniques $\mathscr{L} \rightarrow i_{*}\left(\mathscr{L}_{\mid \mathscr{V}}\right)$ et $\mathscr{O}_{\mathscr{X}} \rightarrow i_{*} \mathscr{O}_{\mathscr{V}}$ sont des isomorphismes. C'est une question locale sur $\mathscr{X}$ pour la topologie lisse, donc en considérant une présentation $\pi: X \rightarrow \mathscr{X}$ de $\mathscr{X}$ qui trivialise $\mathscr{L}$, il suffit de montrer que $\mathscr{O}_{X} \rightarrow j_{*} \mathscr{O}_{V}$ est un isomorphisme, où $j: V \rightarrow X$ est l'immersion ouverte induite par $i$. Par [28] 5.10.5, on est ramené à montrer que tout point $z$ de $Z=X \backslash V$ est de profondeur au moins 2 dans $X$, i.e. que prof $\left(\mathscr{O}_{X, z}\right) \geq 2$ pour tout $z \in Z$. Pour cette question, on peut clairement supposer $X$ affine, et même, vu les considérations ci-dessus, intègre et à fibre spéciale intègre. C'est alors un exercice facile laissé au lecteur.

Remarque 3.2.6 En général, le champ de Picard $\mathscr{P} i c(\mathscr{X} / S)$ n'est pas séparé. Par exemple sous les hypothèses du théorème précédent, $\mathscr{P} i c(\mathscr{X} / S)$ n'est jamais séparé (essentiellement parce que $\mathbb{G}_{\mathrm{m}}$ n'est pas propre).

Remarque 3.2.7 Il est vraisemblable que dans le théorème précédent, supposer les fibres de $\mathscr{X}$ géométriquement normales soit superflu ( $c f$. [14] 8.4 thm. 3).

\section{Autour de l'élément neutre}

\subsection{Lissité et dimension}

Soient $k$ un corps, $P$ un $k$-espace algébrique, et $y:$ Spec $k \rightarrow P$ un $k$-point de $P$. Soit $k[\varepsilon]=k[X] /\left(X^{2}\right)$. On note $i$ l'immersion fermée de $\operatorname{Spec} k$ dans $\operatorname{Spec} k[\varepsilon]$, et $T_{y} P$ l'espace tangent à $P$ en $y$.

$$
T_{y} P=\{\varphi: \operatorname{Spec} k[\varepsilon] \rightarrow P \mid \varphi \circ i=y\}
$$

Si $f: X \rightarrow P$ est un morphisme de $k$-espaces algébriques, et si $x$ est un $k$-point de $X$, on note $T_{x} f: T_{x} X \rightarrow T_{f(x)} P$ le morphisme naturel $\varphi \mapsto f \circ \varphi$. Nous rappelons ci-dessous quelques résultats bien utiles.

Lemme 4.1.1 1) Soit $f: X \rightarrow P$ un morphisme formellement lisse (resp. formellement non ramifié, formellement étale) de $k$-espaces algébriques et soit $x$ : Spec $k \rightarrow X$ un point k-rationnel de $X$. Alors $T_{x} f: T_{x} X \rightarrow T_{f(x)} P$ est surjective (resp. injective, resp. un isomorphisme).

2) Soient $X$ un k-espace algébrique localement de type fini, $x$ un k-point de $X$, et $L$ une extension de $k$. On note $X_{L}$ le L-espace algébrique obtenu par changement de base, et $x_{L}$ le point de $X_{L}$ induit par $x$. Alors le morphisme naturel

$$
\left(T_{x} X\right) \otimes_{k} L \longrightarrow T_{x_{L}}\left(X_{L}\right)
$$

est un isomorphisme. 
Démonstration. Le point 1) résulte immédiatement des définitions. Pour le second point, on se ramène par [32] II (6.4) au cas où $X$ est un schéma affine d'anneau $A$. L'assertion n'est maintenant plus qu'un exercice facile d'algèbre commutative (rédigé en détail dans [15]).

Nous rappelons également le résultat suivant, valable aussi pour un espace algébrique puisqu'un espace algébrique en groupes sur un corps est toujours un schéma.

Proposition 4.1.2 [31, 5.13 et 5.14] Soient $P$ un $k$-schéma en groupes localement de type fini, et e le k-point neutre. Alors $P$ a la même dimension en tout point. De plus cette dimension est inférieure à $\operatorname{dim}_{k}\left(T_{e} P\right)$, et les propriétés suivantes sont équivalentes:

(i) $\operatorname{dim} P=\operatorname{dim}_{k}\left(T_{e} P\right)$;

(ii) $P$ est lisse en 0 ;

(iii) P est lisse.

Elles sont vérifiées lorsque $k$ est de caractéristique nulle.

Théorème 4.1.3 Soient $k$ un corps, $S$ son spectre, et $\mathscr{X}$ un $S$-champ algébrique. On note Pic $\mathscr{X} / k$ le foncteur de Picard relatif Pic $\mathscr{X}_{/ S \text { (Ét) }}$ et on suppose qu'il est représentable par un $S$-espace algébrique localement de type fini.

a) Alors l'espace tangent à l'origine est

$$
T_{0} \operatorname{Pic} \mathscr{X} / k=H^{1}\left(\mathscr{X}, \mathscr{O}_{\mathscr{X}}\right) \text {. }
$$

b) L'espace algébrique $\operatorname{Pic}_{\mathscr{X} / k}$ a la même dimension en tout point. De plus cette dimension est inférieure à $\operatorname{dim}_{k} H^{1}(\mathscr{X}, \mathscr{O} \mathscr{X})$, et il y a égalité si et seulement si $\mathrm{Pic} \mathscr{X} / k$ est lisse à l'origine. Dans ce cas, Pic $\mathscr{X}_{/ k}$ est lisse de dimension $\operatorname{dim}_{k} H^{1}\left(\mathscr{X}, \mathscr{O}_{\mathscr{X}}\right)$ partout. Il en est toujours ainsi lorsque $k$ est de caractéristique nulle.

Démonstration. a) On note encore $P=\operatorname{Pic} \mathscr{X} / k$. L'espace tangent à $P$ en 0 est par définition le noyau de $P(k[\varepsilon]) \rightarrow P(k)$. On note $\widetilde{\mathscr{X}}=\mathscr{X} \otimes_{k} k[\varepsilon]$, et $i$ l'injection canonique de $\mathscr{X}$ dans $\mathscr{X}$. On voit facilement que l'idéal $I$ sur $\mathscr{X}$ de carré nul qui définit $\mathscr{X}$ comme sous-champ fermé de $\widetilde{\mathscr{X}}$ est isomorphe à $i_{*} \mathscr{O} \mathscr{X}$. La remarque 3.1.4 montre alors que le noyau de $\operatorname{Pic}(\widetilde{\mathscr{X}}) \rightarrow \operatorname{Pic}(\mathscr{X})$ s'identifie à $H^{1}\left(\widetilde{\mathscr{X}}, i_{*} \mathscr{O}_{\mathscr{X}}\right)$. Or d'après le lemme A.3.5, $H^{1}\left(\widetilde{\mathscr{X}}, i_{*} \mathscr{O} \mathscr{X}\right)$ est isomorphe à $H^{1}(\mathscr{X}, \mathscr{O} \mathscr{X})$. On a par ailleurs un carré commutatif:

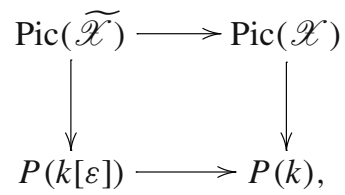

qui induit un morphisme entre les noyaux des flèches horizontales, et donc, d'après ce qui précède, un morphisme $v: H^{1}\left(\mathscr{X}, \mathscr{O}_{\mathscr{X}}\right) \rightarrow T_{0} P$. Montrons que $v$ est un isomorphisme. Remarquons tout d'abord que si $k$ est algébriquement clos, les flèches verticales du carré ci-dessus sont des isomorphismes en vertu de 2.2.1, de sorte que $v$ en est un aussi. Passons maintenant au cas général. Soit $\bar{k}$ une clôture algébrique de 
$k$. Le carré ci-dessus s'envoie alors sur le carré correspondant obtenu après extension du corps de base à $\bar{k}$. On en déduit en regardant les noyaux un carré commutatif de $k$-espaces vectoriels:

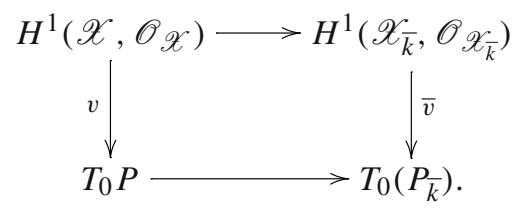

Il résulte du cas précédent que $\bar{v}$ est un isomorphisme. De plus d'après A.3.3 et 4.1.12), $\bar{v}$ s'identifie à $v \otimes_{k} \bar{k}$. On en déduit que $v$ est un isomorphisme. Pour b) il suffit d'appliquer 4.1.2.

\subsection{Construction de la composante neutre}

On rappelle le résultat suivant concernant la composante neutre d'un $k$-schéma en groupes localement de type fini.

Théorème 4.2.1 [31, 5.1] Soient $k$ un corps et $G$ un $k$-schéma en groupes localement de type fini. Alors $G$ est séparé. Soit $G^{0}$ la composante connexe de l'élément neutre de $G$. Alors $G^{0}$ est un sous-schéma en groupes ouvert et fermé de G. Il est de type fini et géométriquement irréductible. De plus, la formation de $G^{0}$ commute aux extensions du corps $k$.

Naturellement, tout ceci est valable pour le foncteur de Picard Pic $\mathscr{X} / k$ dès qu'il est représentable par un espace algébrique localement de type fini sur $k$. En effet, c'est alors automatiquement un schéma en vertu d'un lemme d'Artin [12, lemme 4.2, p. 43]. En particulier, c'est le cas dès que $\mathscr{X}$ est un champ algébrique propre et cohomologiquement plat en dimension zéro sur $k$.

La notion de faisceaux inversibles algébriquement équivalents se généralise très bien aux champs algébriques, et comme dans le cas des schémas elle permet de caractériser les $k$-points du foncteur de Picard qui sont dans la composante neutre.

Définition 4.2.2 ([31, 5.9]) Soit $\mathscr{X}$ un champ algébrique sur un corps $k$. Soient $\mathscr{L}$ et $\mathscr{N}$ deux faisceaux inversibles sur $\mathscr{X}$. On dit que $\mathscr{L}$ et $\mathscr{N}$ sont algébriquement équivalents s'il existe une suite de $k$-schémas connexes de type fini $T_{1}, \ldots, T_{n}$, des points géométriques $s_{i}, t_{i}$ de $T_{i}$ (pour tout $i$ ) ayant tous le même corps, et un faisceau inversible $\mathscr{M}_{i}$ sur $\mathscr{X} \times_{k} T_{i}$ tels que

$$
\mathscr{L}_{s_{1}} \simeq \mathscr{M}_{1, s_{1}}, \quad \mathscr{M}_{1, t_{1}} \simeq \mathscr{M}_{2, s_{2}}, \ldots, \quad \mathscr{M}_{n-1, t_{n-1}} \simeq \mathscr{M}_{n, s_{n}}, \quad \mathscr{M}_{n, t_{n}} \simeq \mathscr{N}_{t_{n}}
$$

Théorème 4.2.3 ([31,5.10]) Soit $\mathscr{X}$ un champ algébrique sur un corps $k$. On suppose que Pic $\mathscr{X}_{/ k}$ est représentable par un schéma localement de type fini. Soit $\mathscr{L}$ un faisceau inversible sur $\mathscr{X}$ et soit $\lambda$ le point correspondant de $\operatorname{Pic}_{\mathscr{X}} / k$. Alors $\mathscr{L}$ est algébriquement équivalent à $\mathscr{O}_{\mathscr{X}}$ si et seulement si $\lambda$ est dans la composante neutre $\operatorname{Pic}_{\mathscr{X} / k}^{0}$. 
Démonstration. On peut recopier telle quelle la démonstration qui accompagne l'énoncé référencé ci-dessus.

Comme toujours, la définition de la composante neutre est plus délicate lorsque la base n'est plus un corps, mais un schéma quelconque. En effet, on dispose, dans chaque fibre $\operatorname{Pic}_{\mathscr{X}_{s} / \kappa(s)}$, d'un ouvert $\operatorname{Pic}_{\mathscr{X}_{s} / \kappa(s)}^{0}$. Mais il se pourrait très bien que la réunion des $\operatorname{Pic}_{\mathscr{X}_{s} / \kappa(s)}^{0}$ ne soit pas un ouvert de $\operatorname{Pic} \mathscr{X} / s$. On peut tout de même définir $a$ priori la composante neutre comme sous-foncteur de $\operatorname{Pic} \mathscr{X} / S$ (voir la remarque 4.2.9).

Définition 4.2.4 Soient $S$ un schéma et $\mathscr{X}$ un $S$-champ algébrique. On suppose que le foncteur de Picard Pic $\mathscr{X} / S$ est représentable par un espace algébrique localement de type fini. On désigne alors par $\operatorname{Pic}_{\mathscr{X} / S}^{0}$ le sous-foncteur de $\mathrm{Pic} \mathscr{X} / S$ défini de la manière suivante. Pour tout $S$-schéma $S^{\prime}$ et pour tout $\xi \in \operatorname{Pic}_{\mathscr{X} / S}\left(S^{\prime}\right)$, on dit que $\xi$ appartient à $\operatorname{Pic}_{\mathscr{X} / S}^{0}\left(S^{\prime}\right)$ si et seulement si pour tout point $s^{\prime}$ de $S^{\prime}$, l'élément $\xi_{\left.\right|_{s^{\prime}}}$ de $\operatorname{Pic} \mathscr{X}_{s^{\prime} / \kappa\left(s^{\prime}\right)}\left(\kappa\left(s^{\prime}\right)\right)$ est dans la composante neutre $\operatorname{Pic}_{\mathscr{X}_{s^{\prime}} / \kappa\left(s^{\prime}\right)}^{0}\left(\kappa\left(s^{\prime}\right)\right)$.

Remarque 4.2.5 Il est clair que $\operatorname{Pic}_{\mathscr{X} / S}^{0}$ est bien un sous-foncteur en groupes de Pic $\mathscr{X} / S$. De plus, la formation de $\operatorname{Pic}_{\mathscr{X} / S}^{0}$ commute au changement de base. Par ailleurs, si $S$ est le spectre d'un corps $k$ et si Pic $\mathscr{X} / k$ est représentable par un schéma en groupes localement de type fini, alors le sous-foncteur $\operatorname{Pic}_{\mathscr{X} / k}^{0}$ ainsi défini coïncide avec le sous-foncteur ouvert défini par la composante connexe de l'élément neutre dans $\operatorname{Pic} \mathscr{X} / k$.

Les lemmes techniques qui suivent ont pour but de généraliser au cas des espaces algébriques un résultat de Grothendieck sur les composantes connexes des fibres le long d'une section (voir 4.2.8).

Lemme 4.2.6 Soient $k$ un corps, $X$ un $k$-schéma connexe localement de type fini, $L$ une extension de $k$ et $p$ la projection de $X_{L}$ dans $X$. Alors les fibres de $p$ rencontrent toutes les composantes connexes de $X_{L}$. Autrement dit, pour toute composante connexe $U$ de $X_{L}$, le morphisme induit $p_{\left.\right|_{U}}: U \rightarrow X$ est surjectif.

Démonstration. En dévissant l'extension, il suffit clairement de traiter le cas d'une extension algébrique et le cas d'une extension transcendante pure. Dans le premier cas, le morphisme $\operatorname{Spec} L \rightarrow$ Spec $k$ est universellement ouvert ([28] 2.4.9) et universellement fermé ([25] 6.1.10). Si $U$ est une composante connexe de $X_{L}$, son image $p(U)$ dans $X$ est ouverte, fermée et non vide, donc c'est $X$ tout entier.

Supposons maintenant l'extension $L / k$ transcendante pure. Si $\Omega$ est une autre extension de $k$, alors l'anneau $L \otimes_{k} \Omega$ est intègre. Il en résulte que les fibres de $p$ sont géométriquement intègres. Notons $\left(X_{i}\right)_{i \in I}$ la famille des composantes connexes de $X_{L}$. Chaque $X_{i}$ est une réunion de fibres, donc les $p\left(X_{i}\right)$ forment une partition de $X$, et ils sont ouverts puisque $p$ est ouvert ([28] 2.4.9). Par connexité un seul d'entre euX est non vide, donc $X_{L}$ est connexe.

Lemme 4.2.7 Soient $V \stackrel{e}{\longrightarrow} X \stackrel{f}{\longrightarrow} S$ deux morphismes de schémas, avec $f$ localement de présentation finie. On note $g=f \circ e$. Si $s \in S$, on note $X_{s}$ la fibre $f^{-1}(s)$ et $X_{s}^{0}(e)$ la réunion des composantes connexes de $X_{s}$ qui rencontrent e $(V)$, et l'on note $X^{0}(e)$ la réunion des $X_{s}^{0}(e)$. (On notera $X_{s}^{0}$ et $X^{0} s^{\prime}$ 'il n'y a pas d'ambiguïté). 
(i) Soit $\varphi: S^{\prime} \rightarrow$ S un morphisme de changement de base. On adopte les notations du diagramme suivant:

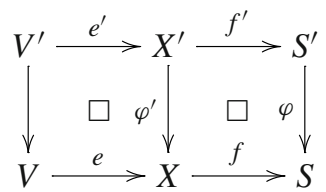

Alors $X^{\prime 0}=\varphi^{\prime-1}\left(X^{0}\right)$.

Dans le cas où $\varphi=g$, le morphisme $f^{\prime}: X^{\prime} \rightarrow S^{\prime}$ a une section $\sigma$. On note $C_{s^{\prime}}^{\prime 0}$ la composante connexe de $\sigma\left(s^{\prime}\right)$ dans $X_{s^{\prime}}^{\prime}$ pour tout $s^{\prime} \in S^{\prime}$, et $C^{\prime 0}$ la réunion $\operatorname{des} C_{s^{\prime}}^{\prime 0}$. Alors $X^{0}=\varphi^{\prime}\left(C^{\prime 0}\right)$.

(ii) On suppose que $f$ est universellement ouvert en tout point de $X^{0}$, et que ses fibres sont géométriquement réduites. On suppose de plus que l'on est dans l'un des deux cas suivants:

a) g est universellement ouvert;

b) e est une immersion ouverte.

Alors $X^{0}$ est un ouvert de $X$.

Démonstration. (i) Pour la première assertion, on peut supposer que $S$ et $S^{\prime}$ sont des spectres de corps. L'inclusion $X^{\prime 0} \subset \varphi^{\prime-1}\left(X^{0}\right)$ est évidente. Réciproquement, soit $x^{\prime} \in X^{\prime}$ tel que $x=\varphi^{\prime}\left(x^{\prime}\right)$ appartienne à $X^{0}$. Soit $C$ la composante connexe de $x$ dans $X$. Par hypothèse il existe un point $v \in V$ tel que $e(v) \in C$. Soit $C^{\prime}$ la composante connexe de $x^{\prime}$ dans $X^{\prime}$. Alors par 4.2.6 on a $\varphi^{\prime}\left(C^{\prime}\right)=C$, donc il existe $x_{1}^{\prime} \in C^{\prime}$ tel que $\varphi^{\prime}\left(x_{1}^{\prime}\right)=e(v)$. Clairement $x_{1}^{\prime}$ est dans l'image de $V^{\prime}$, donc $C^{\prime}$ recontre $e^{\prime}\left(V^{\prime}\right)$ et $x^{\prime}$ appartient à $X^{\prime 0}$.

Pour la seconde assertion, on constate tout d'abord que, vu la construction de $\sigma$, $\sigma\left(S^{\prime}\right)$ est inclus dans $e^{\prime}\left(V^{\prime}\right)$ d'où l'on déduit immédiatement l'inclusion $C^{\prime 0} \subset X^{\prime 0}$, donc $\varphi^{\prime}\left(C^{\prime 0}\right) \subset X^{0}$ grâce à la première assertion. Réciproquement, soit $x \in X^{0}$. On note $s=f(x)$ et $C$ la composante connexe de $x$ dans $X_{s}$. Soit $v \in V$ tel que $e(v) \in C$. On considère le point $s^{\prime}=v$ dans $S^{\prime}=V$. Alors $\varphi\left(s^{\prime}\right)=s$. Soit $C^{\prime}$ la composante connexe de $\sigma\left(s^{\prime}\right)$ dans $X_{s^{\prime}}^{\prime}$. D'après 4.2.6, $\varphi^{\prime}\left(C^{\prime}\right)=C$, ce qui prouve que $x$ appartient à $\varphi^{\prime}\left(C^{\prime 0}\right)$.

(ii) On effectue le changement de base par $g: V \rightarrow S$. Avec les notations de (i), $C^{\prime 0}$ est un ouvert de $X^{\prime}$ par [29] (15.6.4). D'après (i), $X^{0}$ est égal à $\varphi^{\prime}\left(C^{\prime 0}\right)$. On en déduit immédiatement que $X^{0}$ est ouvert dans le cas a).

Dans le cas b), $e$ est universellement ouvert et $f$ l'est en tout point de $e(V)$, donc on est ramené au cas a).

Lemme 4.2.8 Soient $S$ un schéma et $f: X \rightarrow S$ un $S$-espace algébrique localement de présentation finie, à fibres géométriquement réduites, muni d'une section $e: S \rightarrow X$. Pour tout s appartenant à $S$, on note $X_{s}^{0}$ la composante connexe de e $(s)$ dans la fibre $X_{s}$. On note $X^{0}$ la réunion des $X_{s}^{0}$. 
(i) On suppose que $f$ est universellement ouvert en tout point de $X^{0}$ et que pour tout $s, X_{s}^{0}$ est irréductible. Alors $X^{0}$ est un ouvert de $X$.

(ii) On suppose que pour tout $s, X_{s}^{0}$ est géométriquement irréductible, et que la fonction $s \mapsto \operatorname{dim}\left(X_{s}^{0}\right)$ est localement constante sur $S$. Alors $f$ est universellement ouvert en tout point de $X^{0}$. En particulier $X^{0}$ est un ouvert de $X$.

Démonstration. (i) Soit $\pi: X_{1} \rightarrow X$ une présentation de $X$. On note $S_{1}$ le produit fibré $X_{1} \times{ }_{X} S, e_{1}$ la projection de $S_{1}$ sur $X_{1}$ et $f_{1}$ le composé $f \circ \pi$. On applique alors le lemme 4.2.7 au diagramme

$$
S_{1} \stackrel{e_{1}}{\longrightarrow} X_{1} \stackrel{f_{1}}{\longrightarrow} S \text {. }
$$

On vérifie facilement que $\pi$ envoie $X_{1}^{0}\left(e_{1}\right)$ dans $X^{0}$. On en déduit, vu que $\pi$ est étale, que $f_{1}$ est universellement ouvert en tout point de $X_{1}^{0}\left(e_{1}\right)$. Il résulte alors de 4.2.7 (ii), cas a) que $X_{1}^{0}\left(e_{1}\right)$ est ouvert dans $X_{1}$.

On note maintenant $U=\pi\left(X_{1}^{0}\left(e_{1}\right)\right.$ ) (c'est un ouvert de $X$ inclus dans $X^{0}$ ) et $V_{1}=\pi^{-1}(U)$. Autrement dit $V_{1}$ est le saturé de $X_{1}^{0}\left(e_{1}\right)$ pour la relation d'équivalence définie par $\pi$. On note alors $W_{1}=X_{1}^{0}\left(V_{1} \hookrightarrow X_{1}\right)$. En d'autres termes, $W_{1}$ est le sous-ensemble de $X_{1}$ défini de la manière suivante : dans une fibre $X_{1 s}$ au-dessus d'un point $s \in S, W_{1 s}$ est la réunion des composantes connexes de $X_{1 s}$ qui rencontrent $V_{1}$. De même que précédemment on vérifie facilement que $\pi\left(W_{1}\right)$ est inclus dans $X^{0}$, si bien que $f_{1}$ est universellement ouvert en tout point de $W_{1}$. En appliquant le Lemme 4.2.7 (ii), cas b) au diagramme

$$
V_{1} \stackrel{f_{1}}{\longrightarrow} S \text {, }
$$

on en déduit que $W_{1}$ est un ouvert de $X_{1}$. Il suffit donc de montrer que $X^{0}$ est égal à $\pi\left(W_{1}\right)$ pour conclure.

On peut pour cela supposer que $S$ est le spectre d'un corps. De plus, $\pi$ étant surjectif, il suffit de montrer que $\pi^{-1}\left(X^{0}\right)$ est inclus dans $W_{1}$. Maintenant $X^{0}$ est un ouvert irréductible de $X$. Notons $\eta$ son point générique. Vu la construction de $W_{1}$ à partir de $V_{1}$, il suffit de montrer que $V_{1}$ contient le point générique de toute composante irréductible de $\pi^{-1}\left(X^{0}\right)$. Or, $\pi$ étant ouvert, il envoie nécéssairement tous ces points génériques sur $\eta$ d'après [27] (1.10.4). Comme $V_{1}$ est saturé pour la relation d'équivalence définie par $\pi$, il suffit de montrer qu'il contient le point générique d'une composante irréductible de $\pi^{-1}\left(X^{0}\right)$, ce qui est évident puisque c'en est un ouvert non vide.

(ii) La formation de $X^{0}$ commutant au changement de base, il suffit de montrer que $f$ est ouvert en tout point de $X^{0}$. Comme $f$ est localement de présentation finie, il suffit de montrer que $f$ est générisant en tout point de $X^{0}$. Soient $x \in X^{0}$ et $s^{\prime}$ une générisation de $s=f(x)$. On note $x^{\prime}$ le point générique de $X_{s^{\prime}}^{0}$. Il suffit maintenant de montrer que $X_{s}^{0}$ est inclus dans $\overline{X_{s^{\prime}}^{0}}$. 
Or on a les inégalités :

$$
\operatorname{dim}\left(X_{s^{\prime}}^{0}\right) \leq \operatorname{dim}_{e(s)}\left(\overline{X_{s^{\prime}}^{0}} \cap f^{-1}(s)\right) \leq \operatorname{dim}\left(X_{s}^{0}\right)
$$

La première résulte du théorème de semi-continuité de Chevalley ([29] (13.1.3)), compte tenu du fait que $X_{s^{\prime}}^{0}$ est égal à $\overline{X_{s^{\prime}}^{0}} \cap f^{-1}\left(s^{\prime}\right)$. La seconde résulte du fait que toute composante irréductible de $\overline{X_{s^{\prime}}^{0}} \cap f^{-1}(s)$ contenant $e(s)$ est nécessairement incluse dans $X_{s}^{0}$. Si l'on suppose de plus que la fonction $t \mapsto \operatorname{dim}\left(X_{t}^{0}\right)$ est localement constante, alors les membres de gauche et de droite sont égaux, d'où $\operatorname{dim}_{e(s)}\left(\overline{X_{s^{\prime}}^{0}} \cap\right.$ $\left.f^{-1}(s)\right)=\operatorname{dim}\left(X_{s}^{0}\right)$. Maintenant soit $Z$ une composante irréductible de $\overline{X_{s^{\prime}}^{0}} \cap f^{-1}(s)$ contenant $e(s)$ et de dimension $\operatorname{dim}\left(X_{s}^{0}\right)$. Alors $Z$ est nécessairement inclus dans $X_{s}^{0}$, donc $Z=X_{s}^{0}$ puisqu'ils sont de irréductibles et de même dimension, d'où $X_{s}^{0} \subset \overline{X_{s^{\prime}}^{0}}$.

Remarque 4.2.9 Comme sous-foncteur de $X$, l'ouvert $X^{0}$ est caractérisé par la propriété suivante. Pour tout $S$-schéma $T$ et pour tout $\xi \in X(T)$, $\xi$ appartient à $X^{0}(T)$ si et seulement si pour tout $s \in S$, le point $\xi_{s} \in X_{S}\left(T_{S}\right)$ obtenu par changement de base appartient à $X_{s}^{0}\left(T_{s}\right)$, autrement dit le morphisme correspondant $T_{S} \rightarrow X_{S}$ se factorise par $X_{s}^{0}$. Notons aussi que la formation de $X^{0}$ commute au changement de base.

Nous pouvons maintenant énoncer le résultat principal de cette section.

Proposition 4.2.10 Soient $S$ un schéma et $\mathscr{X}$ un $S$-champ algébrique. On suppose que le foncteur de Picard $\operatorname{Pic} \mathscr{X}_{/ S}$ est représentable par un espace algébrique localement de présentation finie sur $S$ et que les fibres $\operatorname{Pic} \mathscr{X}_{s} / \kappa(s)$ sont géométriquement réduites. On suppose de plus que l'une ou l'autre des hypothèses suivantes est vérifiée:

a) le morphisme $\operatorname{Pic} \mathscr{X} / S \rightarrow S$ est universellement ouvert en tout point de $\operatorname{Pic}_{\mathscr{X} / S}^{0}$ (vrai par exemple s'il est plat);

b) la fonction $s \mapsto \operatorname{dim}\left(\mathrm{Pic}_{\mathscr{X}_{s} / \kappa(s)}\right)$ est localement constante sur $S$ (hypothèse vérifiée par exemple si $\operatorname{Pic}_{\mathscr{X}} / \mathrm{S}$ est lisse le long de la section unité).

Alors le morphisme naturel

$$
\operatorname{Pic}_{\mathscr{X} / S}^{0} \longrightarrow \operatorname{Pic} \mathscr{X} / S
$$

est une immersion ouverte. De plus, $\operatorname{Pic}_{\mathscr{X} / S}^{0}$ est de type fini sur $S$.

Démonstration. Que $\operatorname{Pic}_{\mathscr{X} / S}^{0}$ soit un ouvert de Pic $\mathscr{X} / S$ résulte immédiatement du lemme 4.2.8. Pour la dernière assertion il suffit d'appliquer le lemme 4.2.12 ci-dessous.

Remarque 4.2.11 Le lecteur remarquera que dans 4.2.10, la condition b) implique en fait la condition a) (4.2.8 (ii)). 
Lemme 4.2.12 Soient $S$ un schéma et $G$ un S-espace algébrique en groupes localement de type fini et à fibres connexes. Alors le morphisme $\sigma: G \rightarrow S$ est quasicompact.

Démonstration. La question est locale sur $S$ donc on peut supposer $S$ quasi-compact. Maintenant, l'image de la section neutre dans l'espace topologique sous-jacent à $G$ (munie de la topologie induite) est quasi-compacte, donc il existe un ouvert quasicompact $U$ de $G$ qui contient la section neutre. L'espace algébrique $U \times{ }_{S} U$ est quasicompact (on rappelle que $S$ est quasi-séparé) donc pour conclure il suffit de montrer que le morphisme $\alpha: U \times{ }_{S} U \rightarrow G$ défini par $\alpha(g, h)=g h^{-1}$ est surjectif. On peut supposer pour cela que $S$ est le spectre d'un corps algébriquement clos. Si $h$ est un $k$-point de $G$, alors les ouverts $U$ et $h U$ sont non vides, donc s'intersectent puisque $G$ est irréductible (4.2.1). Il existe donc un $k$-point $g$ dans $U$ tel que $h^{-1} g$ appartienne à $U$. Alors $\alpha\left(U, h^{-1} g\right)=U g^{-1} h$ est un voisinage ouvert de $h$. L'image de $\alpha$ contient un ouvert qui contient tout $k$-point, donc elle est égale à $G$.

Exemple 4.2.13 Soit $S$ un schéma de caractéristique zéro et soit $\mathscr{X}$ un $S$-champ algébrique tel que la fonction $s \mapsto \operatorname{dim}_{\kappa(s)} H^{1}\left(\mathscr{X}_{s}, \mathscr{O}_{\mathscr{X}_{s}}\right)$ soit localement constante (il est probable qu' un champ propre et lisse sur $S$ vérifie cette condition, $c f$. [19] (5.5) (i)). On suppose de plus que Pic $\mathscr{X} / S$ est un espace algébrique localement de présentation finie. Alors d'après 4.1.3, les hypothèses de 4.2.10 sont satisfaites.

\subsection{Propreté de la composante neutre}

Théorème 4.3.1 On suppose que $\mathscr{X}$ est un champ algébrique propre, géométriquement normal et cohomologiquement plat en dimension zéro sur Spec $k$. Alors la composante neutre $\operatorname{Pic}_{\mathscr{X} / k}^{0}$ du schéma de Picard est propre sur $k$.

Démonstration. Si $\bar{k}$ est une clôture algébrique de $k$ alors le champ $\mathscr{X}_{\bar{k}}$ obtenu par changement de base est normal d'après [28] (6.7.7). Il vérifie clairement les autres hypothèses du théorème donc par descente fidèlement plate on peut supposer le corps $k$ algébriquement clos.

Il suffit (cf. argumentaire de Kleiman au cours de la démonstration du théorème 5.4 de [31]) de montrer que tout morphisme de schémas de $G$ vers Pic $\mathscr{X} / k$ est constant, avec $G=\mathbb{G}_{a}$ ou $G=\mathbb{G}_{\mathrm{m}}$. (En fait il suffirait même de le faire pour $\mathbb{G}_{\mathrm{m}}$ puisqu'alors c'est aussi vrai pour $G=\mathbb{G}_{a}$, mais cette restriction n'apporte pas grandchose.) En effet, il suffit de montrer que le réduit $\left(\mathrm{Pic}_{\mathscr{X} / k}^{0}\right)$ réd est propre, or ce dernier est lisse donc on peut lui appliquer le théorème de structure de Chevalley et Rosenlicht (cf. par exemple [18], théorème 1.1). On en déduit que $\left(\mathrm{Pic}_{\mathscr{X} / k}^{0}\right)_{\text {réd }}$ a un sous-groupe algébrique linéaire $H$, fermé et distingué dans $\left(\operatorname{Pic}_{\mathscr{X} / k}^{0}\right)_{\text {réd }}$, tel que le quotient $\left(\mathrm{Pic}_{\mathscr{X} / k}^{0}\right)_{\text {réd }} / H$ soit une variété abélienne. Il suffit de montrer que $H$ est trivial. Le groupe $H$ est commutatif donc résoluble. Il est dès lors triangulable d'après le théorème de Lie-Kolchin. On en déduit que s'il était non trivial, il contiendrait un sous-groupe isomorphe à $\mathbb{G}_{\mathrm{m}}$ ou $\mathbb{G}_{a}$ (voir par exemple le livre de Springer [44], lemme 6.3.4). 
Vu que $\mathscr{X}$ a une section, et vu que les groupes de Picard de $\operatorname{Spec} k$ et de $G$ sont triviaux, dire que tout morphisme de schémas de $G$ vers Pic $\mathscr{X} / k$ est constant revient à dire, grâce au théorème 2.2.6, que le morphisme naturel

$$
\operatorname{Pic}(\mathscr{X}) \longrightarrow \operatorname{Pic}(\mathscr{X} \times G)
$$

est un isomorphisme. (Les produits qui apparaissent dans cette démonstration sont des produits au-dessus de $\operatorname{Spec} k$.) La suite exacte des termes de bas degré associée à la suite spectrale de Leray du morphisme $p: \mathscr{X} \times G \rightarrow \mathscr{X}$ s'écrit:

$$
0 \longrightarrow H^{1}\left(\mathscr{X}, p_{*} \mathbb{G}_{\mathrm{m}}\right) \longrightarrow H^{1}\left(\mathscr{X} \times G, \mathbb{G}_{\mathrm{m}}\right) \longrightarrow H^{0}\left(\mathscr{X}, R^{1} p_{*} \mathbb{G}_{\mathrm{m}}\right) .
$$

Commençons par montrer que le faisceau $R^{1} p_{*} \mathbb{G}_{\mathrm{m}}$ est nul. On sait d'après le calcul des images directes supérieures effectué en annexe, que c'est le faisceau associé au préfaisceau qui à tout ouvert lisse-étale $(U, u)$ de $\mathscr{X}$ associe $H^{1}\left(U \times G, \mathbb{G}_{\mathrm{m}}\right)$. Donc il suffit de montrer que pour tout schéma affine $U$ lisse sur $\mathscr{X}$ et pour tout $\xi \in$ $H^{1}\left(U \times G, \mathbb{G}_{\mathrm{m}}\right)$, il existe une famille couvrante étale $V \rightarrow U$ telle que l'élément $\xi_{\left.\right|_{V}}$ de $H^{1}\left(V \times G, \mathbb{G}_{\mathrm{m}}\right)$ soit nul. Mais pour démontrer ceci il suffit clairement de savoir que le morphisme $\operatorname{Pic}(U) \rightarrow \operatorname{Pic}(U \times G)$ est surjectif. Nous sommes donc ramenés à montrer que si $U$ est un schéma affine normal (que l'on peut aussi supposer intègre, vu que $U$ est de toute manière somme disjointe finie de schémas intègres) sur $\operatorname{Spec} k$, alors $\operatorname{Pic}(U \times G)$ s'identifie à $\operatorname{Pic}(U)$. Ce fait est démontré par Kleiman dans [31], au cours de la démonstration du théorème (5.4).

Calculons maintenant le faisceau $p_{*} \mathbb{G}_{\mathrm{m}}$. Considérons d'abord le cas où $G=\mathbb{G}_{\mathrm{m}}$. Nous allons montrer que $p_{*} \mathbb{G}_{\mathrm{m}}$ s'identifie à $\mathbb{G}_{\mathrm{m}} \times \mathbb{Z}$. Il suffit bien évidemment de vérifier que ces deux faisceaux coïncident sur le site Lis-ét $(\mathscr{X})$. Si $U=\operatorname{Spec} A$ est un schéma affine lisse sur $\mathscr{X}$, il est en particulier normal, donc somme disjointe finie de schémas affines intègres, si bien que l'on peut supposer $U$ intègre. Alors $\mathbb{G}_{\mathrm{m}} \times \mathbb{Z}(U)=A^{\times} \times \mathbb{Z}$. Par ailleurs, vu que $p$ est représentable, on a

$$
p_{*} \mathbb{G}_{\mathrm{m}}(U)=\mathbb{G}_{\mathrm{m}}((\mathscr{X} \times G) \times \mathscr{X} U)=\mathbb{G}_{\mathrm{m}}(U \times G)=A\left[X, X^{-1}\right]^{\times} .
$$

Or lorsque $A$ est intègre, il est clair que les éléments inversibles de l'anneau $A\left[X, X^{-1}\right]$ sont les éléments de la forme $a X^{n}$ avec $a \in A^{\times}$et $n \in \mathbb{Z}$. Dans le cas où $G=\mathbb{G}_{a}$, le lecteur vérifiera facilement que l'on trouve $p_{*} \mathbb{G}_{\mathrm{m}}=\mathbb{G}_{\mathrm{m}}$.

Or le groupe $H^{1}(\mathscr{X}, \mathbb{Z})$ est réduit à zéro d'après le théorème 4.3 .2 ci-dessous. On a alors, que $G$ soit égal à $\mathbb{G}_{a}$ ou $\mathbb{G}_{\mathrm{m}}$,

$$
H^{1}\left(\mathscr{X}, p_{*} \mathbb{G}_{\mathrm{m}}\right)=H^{1}\left(\mathscr{X}, \mathbb{G}_{\mathrm{m}}\right)=\operatorname{Pic}(\mathscr{X})
$$

ce qui, vu la suite exacte évoquée ci-dessus, fournit l'isomorphisme désiré.

Théorème 4.3.2 Soit $\mathscr{X}$ un champ algébrique localement noethérien et normal. Alors le groupe $H^{1}(\mathscr{X}, \mathbb{Z})$ est nul. 
Nous allons pour cela montrer que tout $\mathbb{Z}$-torseur sur $\mathscr{X}$ est trivial. Notre démarche est fortement inspirée de l'étude des préschémas constants tordus quasi-isotriviaux proposée dans SGA3 ([1] exposé X, paragraphe 5).

Lemme 4.3.3 Soit $f: \mathscr{X} \rightarrow \mathscr{Y}$ un morphisme représentable de S-champs algébriques. Soit y un point de l'espace topologique $|\mathscr{Y}|$ sous-jacent à $\mathscr{Y}$. Les propositions suivantes sont équivalentes:

(i) Pour un représentant $\operatorname{Spec} K \rightarrow \mathscr{Y}$ de $y$, le morphisme $f_{K}$ induit par changement de base de $\mathscr{X}_{K}=\mathscr{X} \times \mathscr{Y}$ Spec $K$ vers $\operatorname{Spec} K$ est fini.

(ii) Pour tout représentant $\operatorname{Spec} K \rightarrow \mathscr{Y}$ de y, le morphisme $f_{K}$ est fini.

Lorsqu'elles sont vérifiées, on dit que $f_{y}: \mathscr{X}_{y} \rightarrow y$ est fini, ou encore que $\mathscr{X}_{y}$ est fini.

Démonstration. Il suffit clairement de montrer que si $L$ est une extension de $K$ et si $f_{L}$ est fini, alors $f_{K}$ est fini. Or si $f_{L}$ est fini, $\mathscr{X}_{L}$ est un schéma affine, et $\mathscr{X}_{K}$ aussi par descente $f p q c$ pour les morphismes affines. Maintenant la descente fidèlement plate pour les morphismes finis de schémas ([28] (2.7.1)) assure que $f_{K}$ est fini.

Le lemme suivant généralise le lemme 5.13 de [1], exposé $\mathrm{X}$.

Lemme 4.3.4 Soit $p: \mathscr{P} \rightarrow \mathscr{X}$ un morphisme représentable de S-champs algébriques, avec $\mathscr{X}$ localement noethérien. On suppose qu'il existe une présentation $X \rightarrow \mathscr{X}$ de $\mathscr{X}$ telle que $P=\mathscr{P} \times \mathscr{X} X$ soit une union disjointe de copies de $X$. Soit $\mathscr{C}$ un sous-champ ouvert et fermé de $\mathscr{P}$. Alors l'ensemble des points $x$ de $|\mathscr{X}|$ tels que $\mathscr{C}_{x}$ soit fini est ouvert et fermé dans $|\mathscr{X}|$. Si on note $\mathscr{U}$ le sous-champ ouvert et fermé que cet ensemble définit, le champ $\mathscr{C}_{\mathscr{U}}=\mathscr{C} \times \mathscr{X} \mathscr{U}$ est fini sur $\mathscr{U}$.

Démonstration. Notons $C$ le sous-espace algébrique ouvert et fermé de $P$ obtenu par changement de base à partir de $\mathscr{C}$. Les propriétés que l'on veut montrer sont clairement de nature locale pour la topologie lisse sur $\mathscr{X}$, donc il suffit de montrer qu'elles sont vérifiées par $C \rightarrow P \rightarrow X$. Comme $X$ est lui aussi localement noethérien, ses composantes connexes sont ouvertes et fermées donc on peut supposer que $X$ est un schéma connexe. Dans ce cas, vu que $P$ est une union disjointe de copies de $X$, le sous-schéma ouvert et fermé $C$ est lui-même l'union disjointe de certaines de ces copies. Si elles sont en nombre fini alors $C$ est fini sur $X$, sinon l'ensemble des points $x$ de $X$ où $C_{x}$ est fini est vide.

Démonstration du théorème 4.3.2 Nous allons utiliser la description du premier groupe de cohomologie en termes de torseurs (cf. paragraphe A.3). Il suffit en vertu de A.3.10 de montrer que tout $\mathbb{Z}$-torseur sur $\mathscr{X}$ au sens de A.3.9 est trivial. Le cas où $\mathscr{X}$ est le spectre d'un corps est bien connu et nous nous en servirons par la suite. Remarquons tout d'abord que dans tous les champs algébriques (a fortiori tous les schémas ou espaces algébriques) qui vont intervenir au cours de la démonstration, les composantes connexes sont irréductibles. En effet, ils seront tous normaux et localement noethériens car ce sont là des propriétés de nature locale pour la topologie lisse. Notre affirmation résulte alors de la proposition 4.13 de [34].

Donnons-nous donc un $\mathbb{Z}$-torseur sur $\mathscr{X}$, c'est-à-dire un morphisme $p: \mathscr{P} \rightarrow \mathscr{X}$ représentable et lisse muni d'une action de $\mathbb{Z}$ qui en fait un torseur (cf. A.3.9). Pour 
montrer que $\mathscr{P}$ est trivial, on peut supposer $\mathscr{X}$ connexe donc irréductible. Soit $\mathscr{C}$ une composante connexe de $\mathscr{P}$. Notons $\eta$ le point générique de l'espace $|\mathscr{X}|$ sous-jacent à $\mathscr{X}$.

- La fibre générique $\mathscr{C}_{\eta}$ est finie.

Soit $u: U \rightarrow \mathscr{X}$ un morphisme lisse, où $U$ est un schéma affine irréductible (donc intègre, puisque $U$ est normal). On note $s$ le point générique de $U$ et on adopte encore les notations du diagramme suivant:

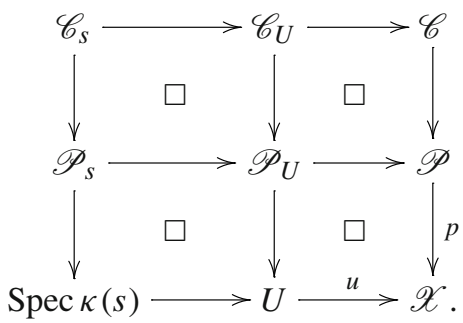

Notons $\left(\mathscr{C}_{i}\right)_{i \in I}$ les composantes connexes de $\mathscr{C}_{U}$ et pour chaque $i \in I$ notons $\eta_{i}$ le point générique de $\left|\mathscr{C}_{i}\right|$. Notons enfin $\xi$ le point générique de $|\mathscr{C}|$ et $\operatorname{Spec} K \rightarrow \mathscr{C}$ l'un de ses représentants. Pour tout $i$ le morphisme de $\mathscr{C}_{i}$ vers $\mathscr{C}$ est lisse donc générisant ([34] (5.8)) si bien qu'il envoie le point $\eta_{i}$ sur $\xi$. On en déduit (cf. par exemple [34] (5.4) (iv)) que le champ $\mathscr{C}_{i, K}=\mathscr{C}_{i} \times \mathscr{C}_{C}$ Spec $K$ est non vide. Maintenant, les $\left|\mathscr{C}_{i, K}\right|$ forment une partition ouverte de $\left|\mathscr{C}_{U, K}\right|$, où $\mathscr{C}_{U, K}$ est le produit fibré $\mathscr{C}_{U} \times \mathscr{C}_{S} \operatorname{Spec} K$. Or le champ

$$
\mathscr{C}_{U, K}=U \times \mathscr{X} \operatorname{Spec} K
$$

est quasi-compact car $\mathscr{X}$ est quasi-séparé donc les $\left|\mathscr{C}_{i, K}\right|$ sont en nombre fini et finalement $\mathscr{C}_{U}$ n'a qu'un nombre fini de composantes connexes.

Par ailleurs, pour chaque $i$ le morphisme naturel de $\mathscr{C}_{i}$ vers $U$ est lui aussi générisant donc il envoie $\eta_{i}$ sur $s$. En particulier sa fibre générique est irréductible donc $\mathscr{C}_{s}$ est une union finie d'irréductibles. Comme $\mathscr{P}_{S}$ est un $\mathbb{Z}$-torseur sur un corps, il est nécessairement trivial, donc $\mathscr{C}_{s}$ est une union disjointe finie de copies $\operatorname{de} \operatorname{Spec} \kappa(s)$. Or le morphisme de $\operatorname{Spec} \kappa(s)$ vers $\mathscr{X}$ est un représentant de $\eta$ donc $\mathscr{C}_{\eta}$ est fini.

- Montrons que $\mathscr{C}$ est fini sur $\mathscr{X}$.

D'après le lemme 4.3.4, l'ensemble des points $x$ de $|\mathscr{X}|$ où $\mathscr{C}_{x}$ est fini est ouvert et fermé dans $|\mathscr{X}|$. Or il est non vide puisqu'il contient $\eta$, donc par connexité c'est $|\mathscr{X}|$ tout entier. Le même lemme prouve alors que le morphisme de $\mathscr{C}$ vers $\mathscr{X}$ est fini.

- Montrons que $\mathscr{C} \rightarrow \mathscr{X}$ est étale.

Il s'agit d'un morphisme fini. En particulier il est schématique. Notre assertion résulte alors du fait qu'un morphisme fini et lisse de schémas est étale ([5] II 1.4).

- Montrons que $\mathscr{C} \rightarrow \mathscr{X}$ est radiciel.

Supposons qu'il existe un corps $K$ et un morphisme Spec $K \rightarrow \mathscr{X}$ tel que le schéma $\mathscr{C}_{K}$ obtenu par changement de base contienne au moins deux points $x_{1}$ et $x_{2}$. Notons 
$c_{1}$ et $c_{2}$ leurs images dans $|\mathscr{C}| \subset|\mathscr{P}|$. Soit $n$ l'unique élément de $\mathbb{Z} \backslash\{0\}$ tel que l'automorphisme $\tau_{n}$ correspondant envoie $x_{1}$ sur $x_{2}$. On note encore $\tau_{n}$ l'automorphisme de $|\mathscr{P}|$ qui correspond à $n$. Il est clair qu'il envoie $c_{1}$ sur $c_{2}$. Or $\tau_{n}(|\mathscr{C}|)$ est un connexe qui contient $c_{2}$ donc il est inclus dans la composante connexe de $c_{2}$, à savoir $|\mathscr{C}|$. On montre de même que $\tau_{n}^{-1}(|\mathscr{C}|)$ est inclus dans $|\mathscr{C}|$ donc $\tau_{n}$ induit un automorphisme de $|\mathscr{C}|$ et tous les $\tau_{n}^{k}\left(c_{1}\right), k \in \mathbb{Z}$, sont dans $|\mathscr{C}|$. Donc tous les $\tau_{n}^{k}\left(x_{1}\right)$ sont dans l'ouvert $\mathscr{C}_{K}$ de $\mathscr{P}_{K}$, ce qui contredit le fait qu'il est de type fini.

\section{- Conclusion.}

Le morphisme de $\mathscr{C}$ vers $\mathscr{X}$ est schématique, étale, radiciel et de type fini donc par [5] I 5.1 c'est une immersion ouverte. De plus il est aussi fermé puisqu'il est fini donc par connexité de $\mathscr{X}$ il est surjectif. Ceci prouve que c'est un isomorphisme, donc le torseur $\mathscr{P} \rightarrow \mathscr{X}$ est trivial.

Théorème 4.3.5 Soit $\mathscr{X}$ un $S$-champ algébrique. On suppose que $\mathrm{Pic} \mathscr{X} / S$ est représentable par un espace algébrique localement de présentation finie, que $\mathrm{Pic}_{\mathscr{X} / S}^{0}$ est un ouvert de $\operatorname{Pic} \mathscr{X} / S$ et qu'il est de plus séparé et de type fini sur $S$. Alors l'ensemble $U$ des points $s$ de $S$ tels que $\mathrm{Pic}_{\mathscr{X}_{s} / \kappa(s)}^{0}$ soit propre sur $\kappa(s)$ est un ouvert de $S$. De plus $\operatorname{Pic}_{\mathscr{X} / S}^{0} \times{ }_{S} U$ est propre sur $U$.

Démonstration. On note $P$ l'espace algébrique $\operatorname{Pic}_{\mathscr{X} / S}^{0}$ et $f$ son morphisme structural vers $S$. Des arguments standard de passage à la limite permettent de supposer $S$ noethérien. Il faut montrer que tout point $s$ de $S$ tel que $P_{S}$ soit propre sur $\kappa(s)$ admet un voisinage ouvert $U$ tel que le morphisme induit $f^{-1}(U) \rightarrow U$ soit propre. D'après [29] 8.10 .5 (xii) (combiné avec le lemme de Chow pour les espaces algébriques), on peut supposer que $S$ est le spectre d'un anneau local noethérien $A$, et il faut montrer que $f$ est propre dès que la fibre du point fermé l'est. Par un argument de descente fidèlement plate, on peut même supposer $A$ complet. Nous montrons d'abord l'assertion suivante.

- Assertion : Si $f: P \rightarrow$ S est un morphisme séparé et de type fini d'espaces algébriques (où $S$ est le spectre d'un anneau local noethérien complet) et si la fibre spéciale de $f$ est propre, alors $P$ admet un sous-espace ouvert et fermé $P_{0}$ qui est propre sur $S$ et qui contient la fibre spéciale.

On peut clairement supposer la fibre spéciale non vide. Commençons par traiter le cas où $P$ est irréductible. En utilisant le lemme de Chow pour les espaces algébriques ([32] IV 3.1), on peut trouver un schéma irréductible $V$ et un morphisme surjectif et projectif $g$ de $V$ vers $P$. Maintenant, vu que la fibre spéciale de $P$ est propre, celle de $V$ l'est aussi. D'après [26] 5.5.2, on en déduit que $V$ lui-même est propre sur $S$, ce qui prouve que $P$ est propre puisqu'il est déjà séparé et de type fini et que $g$ est surjectif.

Supposons maintenant $P$ connexe. On le décompose alors en composantes irréductibles $P_{1}, \ldots, P_{n}$. Soit $Z$ la réunion des composantes qui rencontrent la fibre spéciale. Chacune de ces composantes est propre d'après le cas précédent, si bien que $Z$ est propre. De plus, $Z$ est non vide puisque la fibre spéciale est supposée non vide. Soit $F$ la réunion des autres composantes. Supposons $F$ non vide. Alors $F$ rencontre $Z$ puisque $P$ est connexe, et le produit fibré $Z \times{ }_{P} F$ est propre sur $S$. En particulier, son 
image est un fermé non vide de $S$, donc elle contient le point fermé. Ceci prouve que $F$ rencontre la fibre spéciale, ce qui est absurde. Donc $F$ est vide et finalement $P$ est propre.

Pour prouver l'assertion dans le cas général, il suffit d' appliquer le résultat à chacune des composantes connexes de $P$, puis de regrouper celles qui rencontrent la fibre spéciale d'une part, et les autres d'autre part.

\section{- Conclusion}

D'après l'assertion précédente $P$ s'écrit comme l'union disjointe d'un sous-espace propre $P_{0}$ et d'un sous-espace $P_{1}$ qui s'envoie dans le complémentaire du point fermé. Vu que $S$ est connexe, l'image de la section neutre est entièrement contenue dans $P_{0}$. Mais comme par ailleurs les fibres de $f$ sont connexes, le fait qu'elles rencontrent $P_{0}$ montre qu'elles sont incluses dans $P_{0}$, si bien que $P_{1}$ est vide et que $P$ est propre.

Exemple 4.3.6 Sous réserve que l'on dispose pour les champs algébriques d'un analogue de [19] 5.5, si $S$ est de caractéristique zéro, et si $\mathscr{X}$ est un $S$-champ algébrique propre, lisse et à fibres géométriquement connexes, alors $\mathrm{Pic} \mathscr{X} / S$ est un espace algébrique localement de présentation finie et séparé, et $\operatorname{Pic}_{\mathscr{X} / S}^{0}$ est un ouvert de $\mathrm{Pic}_{\mathscr{X}} / S$ qui est propre sur $S$ ( $c f .3 .2 .5,4.2 .13,4.3 .1$ et 4.3.5).

\section{Quelques exemples}

\subsection{Espace de module des courbes elliptiques}

Soient $S$ un schéma et $\mathscr{M}_{1,1, S}$ le $S$-champ qui classifie les courbes elliptiques. Mumford a calculé en 1965 le groupe de Picard de $\mathscr{M}_{1,1, S}$ lorsque $S$ est le spectre d'un corps de caractéristique différente de 2 et 3 . Ce groupe est isomorphe à $\mathbb{Z} / 12 \mathbb{Z}$, engendré par le fibré de Hodge $\lambda$ défini de la manière suivante. Si $t: T \rightarrow \mathscr{M}_{1,1, S}$ est un $T$-point de $\mathscr{M}_{1,1, S}$ correspondant à une courbe elliptique $f: E \rightarrow T$, la restriction $t^{*} \lambda$ est le faisceau inversible $f_{*} \Omega_{E / T}$. Fulton et Olsson généralisent ce résultat dans [22]. Ils montrent que si la base $S$ est réduite ou est un $\mathbb{Z}\left[\frac{1}{2}\right]$-schéma, alors le groupe $\operatorname{Pic}\left(\mathscr{M}_{1,1, S}\right)$ s'identifie à

$$
\operatorname{Pic}\left(\mathbb{A}_{S}^{1}\right) \times \mathbb{Z} / 12 \mathbb{Z}(S)
$$

On en déduit que le foncteur de Picard Pic $\mathscr{M}_{1,1, S} / S$ n'est pas représentable. En effet, dans le cas contraire, le foncteur de Picard de la droite affine serait représentable, ${ }^{6}$ ce que l'on sait être faux.

Fulton et Olsson calculent aussi le groupe de Picard de la compactification standard $\overline{\mathscr{M}}_{1,1, S}$ de $\mathscr{M}_{1,1, S}$. Il est isomorphe à $\mathbb{Z}(S) \times \operatorname{Pic}(S)$. On en déduit immédiatement que le foncteur de Picard de $\overline{\mathscr{M}_{1,1, S}}$ est représentable, isomorphe à $\mathbb{Z}$, et que son champ de Picard est isomorphe à $\mathbb{Z} \times \mathrm{B} \mathbb{G}_{\mathrm{m}}$.

\footnotetext{
6 au moins dans le cas où $S$ est un $\mathbb{Z}\left[\frac{1}{2}\right]$-schéma. Pour le cas général, on obtient aussi une contradiction car pour montrer que $\mathrm{Pic}_{\mathbb{A}_{S}} / S$ n'est pas représentable, il suffit de considérer des schémas de base réduits.
} 
5.2 Espaces projectifs à poids

Définition 5.2.1 Soient $n \geq 1$ et $a_{0}, \ldots, a_{n}$ des entiers strictement positifs. Le groupe $\mathbb{G}_{\mathrm{m}}$ agit sur $\mathbb{A}_{\mathbb{Z}}^{n+1} \backslash\{0\}$ par

$$
\lambda \cdot\left(x_{0}, \ldots, x_{n}\right)=\left(\lambda^{a_{0}} x_{0}, \ldots, \lambda^{a_{n}} x_{n}\right) .
$$

On note $\mathbb{P}\left(a_{0}, \ldots, a_{n}\right)$ le champ quotient $\left[\mathbb{A}_{\mathbb{Z}}^{n+1} \backslash\{0\} / \mathbb{G}_{\mathrm{m}}\right]$ de $\mathbb{A}_{\mathbb{Z}}^{n+1} \backslash\{0\}$ par cette action, et on l'appelle espace projectif de poids $\left(a_{0}, \ldots, a_{n}\right)$.

Nous allons voir que la théorie développée ci-dessus permet de retrouver très rapidement le résultat de [39], à savoir que le champ de Picard de $\mathbb{P}\left(a_{0}, \ldots, a_{n}\right)$ est isomorphe à $\mathbb{Z} \times \mathrm{B} \mathbb{G}_{\mathrm{m}}$. On note $\mathscr{X}=\mathbb{P}\left(a_{0}, \ldots, a_{n}\right)$ et $\mathscr{X}_{T}=\mathscr{X} \times T$ pour tout schéma $T$. Il est bien connu que $\mathscr{X}$ est propre, plat et cohomologiquement plat sur $S=\operatorname{Spec} \mathbb{Z}$, donc on sait déjà que Pic $\mathscr{X} / S$ est représentable par un espace algébrique localement séparé. De plus $\mathscr{X} \rightarrow S$ a une section donc son champ de Picard est isomorphe à Pic $\mathscr{X} / S \times \mathrm{B} \mathbb{G}_{\mathrm{m}}$ et il suffit de voir que Pic $\mathscr{X} / S$ est isomorphe à $\mathbb{Z}$.

Lemme 5.2.2 Le morphisme $\mathrm{Pic} \mathscr{X} / S \rightarrow S$ est non ramifié.

Démonstration. Il est déjà localement de présentation finie. Il suffit donc de montrer que pour tout schéma affine $S^{\prime}$, tout sous-schéma fermé $S_{0}^{\prime}$ de $S^{\prime}$ défini par un idéal $I$ de carré nul, et tout morphisme de $S^{\prime}$ dans $S$, l'application canonique de

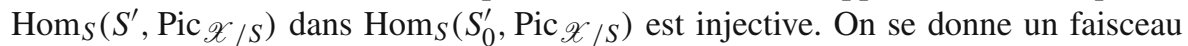
inversible $\mathscr{L}$ sur $\mathscr{X}_{S^{\prime}}$ dont la restriction $\mathscr{L}_{0}$ à $\mathscr{X}_{S_{0}^{\prime}}$ provient de la base $S_{0}^{\prime}$. Il faut montrer que $\mathscr{L}$ provient de la base $S^{\prime}$. Par hypothèse, $\mathscr{L}_{0}$ provient d'un faisceau $\mathscr{B}_{0}$ sur $S_{0}^{\prime}$, qui lui-même provient d'un faisceau inversible $\mathscr{B}$ sur $S^{\prime}$ puisque $H^{2}\left(S^{\prime}, I\right)$ est nul (on utilise 3.1.3). Maintenant $\mathscr{L}$ et $\mathscr{B}_{\mid \mathscr{X}_{S^{\prime}}}$ sont deux déformations de $\mathscr{L}_{0}$ à $\mathscr{X}_{S^{\prime}}$. D'après le théorème 3.1 .3 , pour montrer qu'elles sont isomorphes il suffit de montrer que le groupe $H^{1}\left(\mathscr{X}_{S^{\prime}}, I\right)$ est nul. Or en considérant la suite exacte longue de cohomologie associée à la suite exacte courte

$$
0 \longrightarrow I \longrightarrow \mathscr{O}_{\mathscr{X}_{S^{\prime}}} \longrightarrow i_{*} \mathscr{O}_{\mathscr{X}_{S_{0}^{\prime}}} \longrightarrow 0
$$

on voit que le groupe $H^{1}\left(\mathscr{X}_{S^{\prime}}, I\right)$ s'injecte dans $H^{1}\left(\mathscr{X}_{S^{\prime}}, \mathscr{O} \mathscr{X}_{S^{\prime}}\right)$ donc il suffit d'appliquer le lemme suivant.

Lemme 5.2.3 Pour tout $S^{\prime}$ affine, le groupe $H^{1}\left(\mathscr{X}_{S^{\prime}}, \mathscr{O}_{\mathscr{X}_{S^{\prime}}}\right)$ est nul.

Démonstration. Nous allons faire le calcul «à la Čech ». On considère la présentation $\pi: X=\mathbb{A}_{S^{\prime}}^{n+1} \backslash\{0\} \rightarrow \mathscr{X}_{S^{\prime}}$ de $\mathscr{X}_{S^{\prime}}$. La suite spectrale A.2.1 associée à ce recouvrement induit la suite exacte longue en bas degrés (avec les notations de A.2.1):

$$
0 \longrightarrow \check{H}^{1}\left(H^{0}\left(X^{\bullet}, \mathscr{O}_{X}^{\bullet}\right)\right) \longrightarrow H^{1}\left(\mathscr{X}_{S^{\prime}}, \mathscr{O}_{\mathscr{X}_{S^{\prime}}}\right) \longrightarrow \check{H}^{0}\left(H^{1}\left(X^{\bullet}, \mathscr{O}_{X}^{\bullet}\right)\right)
$$


Donc pour conclure il suffit de montrer que le groupe de gauche et le groupe de droite sont nuls. En identifiant $X \times \mathscr{X}_{S^{\prime}} X$ à $X \times{ }_{S^{\prime}} \mathbb{G}_{\mathrm{m}}$ et $X \times \mathscr{X}_{S^{\prime}} X \times \mathscr{X}_{S^{\prime}} X$ à $X \times{ }_{S^{\prime}} \mathbb{G}_{\mathrm{m}} \times{ }_{S^{\prime}} \mathbb{G}_{\mathrm{m}}$, le premier de ces groupes est l'homologie du complexe

$$
\begin{aligned}
& H^{0}\left(X, \mathscr{O}_{X}\right) \stackrel{p_{1}^{*}-p_{2}^{*}}{\longrightarrow} H^{0}\left(X \times_{S^{\prime}} \mathbb{G}_{\mathrm{m}}, \mathscr{O}_{X \times_{S^{\prime}} \mathbb{G}_{\mathrm{m}}}\right) \\
& \stackrel{p_{23}^{*}-p_{13}^{*}+p_{12}^{*}}{\longrightarrow} H^{0}\left(X \times_{S^{\prime}} \mathbb{G}_{\mathrm{m}} \times_{S^{\prime}} \mathbb{G}_{\mathrm{m}}, \mathscr{O}_{X \times_{S^{\prime}}} \mathbb{G}_{\mathrm{m}} \times_{S^{\prime}} \mathbb{G}_{\mathrm{m}}\right)
\end{aligned}
$$

où les morphismes $p_{i}: X \times_{S^{\prime}} \mathbb{G}_{\mathrm{m}} \rightarrow X$ et $p_{i j}: X \times{ }_{S^{\prime}} \mathbb{G}_{\mathrm{m}} \times{ }_{S^{\prime}} \mathbb{G}_{\mathrm{m}} \rightarrow X \times{ }_{S^{\prime}} \mathbb{G}_{\mathrm{m}}$ sont donnés par:

$$
\begin{aligned}
& p_{1}:(x, g) \mapsto x \\
& p_{2}:(x, g) \mapsto x \cdot g \\
& p_{12}:(x, g, h) \mapsto(x, g) \\
& p_{13}:(x, g, h) \mapsto(x, g h) \\
& p_{23}:(x, g, h) \mapsto(x . g, h)
\end{aligned}
$$

Or les groupes $H^{0}(X), H^{0}\left(X \times_{S^{\prime}} \mathbb{G}_{\mathrm{m}}\right)$ et $H^{0}\left(X \times_{S^{\prime}} \mathbb{G}_{\mathrm{m}} \times{ }_{S^{\prime}} \mathbb{G}_{\mathrm{m}}\right)$ s'identifient respectivement à $H^{0}\left(\mathbb{A}_{S^{\prime}}^{n+1}\right), H^{0}\left(\mathbb{A}_{S^{\prime}}^{n+1} \times{ }_{S^{\prime}} \mathbb{G}_{\mathrm{m}}\right)$ et $H^{0}\left(\mathbb{A}_{S^{\prime}}^{n+1} \times{ }_{S^{\prime}} \mathbb{G}_{\mathrm{m}} \times{ }_{S^{\prime}} \mathbb{G}_{\mathrm{m}}\right)$. On note $A$ l'anneau de $S^{\prime}$ et $\mathbb{A}_{S^{\prime}}^{n+1}=\operatorname{Spec} A\left[x_{0}, \ldots, x_{n}\right]=\operatorname{Spec} A[\underline{x}]$. On note aussi $\underline{x}^{y}$ le $(n+1)$-uplet $\left(x_{0} y^{a_{0}}, \ldots, x_{n} y^{a_{n}}\right)$. Alors l'image de $p_{1}^{*}-p_{2}^{*}$ s'identifie à l'ensemble des polynômes de la forme $P(\underline{x})-P\left(\underline{x}^{y}\right)$ dans $A\left[\underline{x}, y, y^{-1}\right]$, où $P \in A[\underline{x}]$. Le noyau de $p_{23}^{*}-p_{13}^{*}+p_{12}^{*}$ s'identifie à l'ensemble des polynômes $Q$ de $A\left[\underline{x}, y, y^{-1}\right]$ vérifiant l'équation

$$
Q(\underline{x}, y z)=Q(\underline{x}, y)+Q\left(\underline{x}^{y}, z\right) .
$$

On vérifie facilement qu'un tel polynôme est nécessairement dans $A[\underline{x}, y]$, puis qu'il est égal à $P(\underline{x})-P\left(\underline{x}^{y}\right)$ où $P$ est donné par $P(\underline{x})=Q(\underline{x}, 0)$. Ceci montre que le groupe de gauche dans la suite exacte longue considérée ci-dessus est nul. Le calcul pour le groupe de droite est du même tonneau et laissé au lecteur.

Se donner un faisceau inversible sur $\mathscr{X}_{S}$ revient à se donner un faisceau inversible $\mathbb{G}_{\mathrm{m}}$-équivariant sur $\mathbb{A}_{S}^{n+1} \backslash\{0\}$, c'est-à-dire un faisceau inversible $\mathscr{L}$ sur $\mathbb{A}_{S}^{n+1} \backslash\{0\}$ et un élément de $\mathbb{G}_{\mathrm{m}}\left(\mathbb{A}_{S}^{n+1} \backslash\{0\} \times \mathbb{G}_{\mathrm{m}}\right)$ qui vérifie une certaine condition de cocycle. Si $d$ est un entier, l'élément $\lambda^{d}$ de $\mathbb{G}_{\mathrm{m}}\left(\mathbb{G}_{\mathrm{m}}\right)=\mathbb{Z}\left[\lambda, \lambda^{-1}\right]^{\times}$définit un élément de $\mathbb{G}_{\mathrm{m}}\left(\mathbb{A}_{S}^{n+1} \backslash\{0\} \times \mathbb{G}_{\mathrm{m}}\right)$ qui vérifie bien cette condition de cocycle. On note $\mathscr{O}(d)$ le faisceau inversible ainsi construit sur $\mathscr{X}_{S}$. Alors l'application $d \mapsto \mathscr{O}(d)$ définit un morphisme d'espaces algébriques

$$
\varphi: \mathbb{Z} \longrightarrow \operatorname{Pic} \mathscr{X} / S
$$

On va construire son inverse via le degré. Si $S$ est le spectre d'un corps $k$ un calcul élémentaire montre que l'application de $\mathbb{Z}$ dans $\operatorname{Pic}\left(\mathscr{X}_{k}\right)$ décrite ci-dessus est un isomorphisme. Si $\mathscr{L}$ est un faisceau inversible sur $\mathscr{X}_{k}$, on note $\operatorname{deg}(\mathscr{L})$ l'entier 
correspondant à $\mathscr{L}$. Maintenant si $S$ est une base quelconque et $\mathscr{L}$ un faisceau inversible sur $\mathscr{X}_{S}$, on définit une fonction $\operatorname{deg}_{\mathscr{L}}$ sur $S$ à valeurs dans $\mathbb{Z}$ en associant à chaque point $s$ de $S$ le degré du faisceau $\mathscr{L}_{s}$ sur $\mathscr{X}_{\kappa(s)}$. D'après 5.2.2, la diagonale $\Delta: \operatorname{Pic} \mathscr{X} / S \rightarrow \operatorname{Pic} \mathscr{X} / S \times{ }_{S} \operatorname{Pic} \mathscr{X} / S$ est une immersion ouverte. On en déduit facilement que $\operatorname{deg} \mathscr{L}$ est une fonction localement constante sur $S$. L'application $\mathscr{L} \mapsto \operatorname{deg} \mathscr{L}$ définit donc un morphisme de $\operatorname{Pic}\left(\mathscr{X}_{S}\right)$ dans $\mathbb{Z}(S)$, c'est-à-dire un morphisme de préfaisceaux de $P$ dans le faisceau constant $\mathbb{Z}$, où $P$ est le préfaisceau $S \mapsto \operatorname{Pic}\left(\mathscr{X}_{S}\right)$. Par propriété universelle du faisceau associé à un préfaisceau, on en déduit un morphisme de faisceaux de $\operatorname{Pic} \mathscr{X} / S$ dans $\mathbb{Z}$, dont il est clair qu'il est un inverse de $\varphi$.

\subsection{Racine $n^{\text {ième }}$ d'un faisceau inversible}

Soient $X$ un $S$-schéma et $\mathscr{L}$ un faisceau inversible sur $X$. Soit $n$ un entier strictement positif. On fabrique un champ $\left[\mathscr{L}^{\frac{1}{n}}\right]$ à partir de ces données de la manière suivante. Si $U$ est un objet de $(\mathrm{Aff} / \mathrm{S}),\left[\mathscr{L}^{\frac{1}{n}}\right]_{U}$ est la catégorie des triplets $(x, \mathscr{M}, \varphi)$ où

$$
\left\{\begin{array}{l}
x: U \rightarrow X \text { est un élément de } X(U) \\
\mathscr{M} \text { est un faisceau inversible sur } \mathrm{U} \\
\varphi: \mathscr{M}^{\otimes n} \rightarrow x^{*} \mathscr{L} \text { est un isomorphisme de faisceaux inversibles. }
\end{array}\right.
$$

On note $\pi$ le morphisme canonique de $\left[\mathscr{L}^{\frac{1}{n}}\right]$ dans $X$. Si $U \in$ ob (Aff/S) et si $\alpha$ est un objet de $\left[\mathscr{L}^{\frac{1}{n}}\right]_{U}$, le foncteur $\mathscr{A} u t{ }_{U}(\alpha)$ est représentable par $\mu_{n}$. Plus généralement, si $\alpha_{1}, \alpha_{2}$ sont deux objets de $\left[\mathscr{L}^{\frac{1}{n}}\right]_{U}$, alors le foncteur $\mathscr{I}_{\text {Som }}\left(\alpha_{1}, \alpha_{2}\right)$ est représentable par un schéma fini sur $U$ (localement ce schéma est de la forme $\operatorname{Spec}\left(A[X] /\left(X^{n}-\gamma\right)\right.$ ) où Spec $A$ est un ouvert de $U$ qui trivialise les objets $\alpha_{1}$ et $\alpha_{2}$ et $\gamma$ est un élément de $A^{\times}$). En d'autres termes le morphisme diagonal $\Delta_{\pi}$ est schématique et fini. En particulier $\left[\mathscr{L}^{\frac{1}{n}}\right]$ est un $S$-préchamp. Il est clair que c'est même un $S$-champfppf. Si $\psi: Y \rightarrow X$ est un morphisme de $S$-schémas, alors le $S$-champ $\left[\mathscr{L}^{\frac{1}{n}}\right] \times_{X} Y$ est canoniquement 1-isomorphe à $\left[\left(\psi^{*} \mathscr{L}\right)^{\frac{1}{n}}\right]$.

Proposition 5.3.1 1) Le champ $\left[\mathscr{L}^{\frac{1}{n}}\right]$ est une gerbe pour la topologie fppf sur $X$. Si S est un $\mathbb{Z}\left[\frac{1}{n}\right]$-schéma, alors $\left[\mathscr{L}^{\frac{1}{n}}\right]$ est même une gerbe pour la topologie étale.

2) Si $\mathscr{L}$ a une racine $n^{\text {ième }}$ sur $X$ alors $\left[\mathscr{L}^{\frac{1}{n}}\right]$ est canoniquement (une fois qu'on a fixé un faisceau inversible $\mathscr{M}$ et un isomorphisme entre $\mathscr{M}^{\otimes n}$ et $\mathscr{L}$ ) 1-isomorphe au champ classifiant $\left(\mathrm{B} \mu_{n}\right)_{\mathrm{fppf}} d u$ groupe $\mu_{n}$ pour la topologie fppf. En particulier c'est un champ algébrique ([34] (10.6) et (10.13.1)).

3) Le champ $\left[\mathscr{L}^{\frac{1}{n}}\right]$ est algébrique.

Démonstration. 1) Il est clair que $\left[\mathscr{L}^{\frac{1}{n}}\right]$ a des objets partout localement pour la topologie de Zariski, donc $\pi$ est un épimorphisme. Pour montrer que le morphisme diagonal est un épimorphisme on est ramené à montrer que pour tout schéma affine Spec $A$, tout élément de $A^{\times}$admet une racine $n^{\text {ième }}$ localement pour la topologie 
considérée. Si $\gamma \in A^{\times}$, le morphisme de Spec $A[X] /\left(X^{n}-\gamma\right)$ vers Spec $A$ est une famille couvrante pour la topologie fppf qui répond au problème posé. Si de plus $n$ est inversible alors c'est même une famille couvrante pour la topologie étale.

2) La donnée d'un faisceau inversible $\mathscr{M}$ sur $X$ et d'un isomorphisme $\varphi$ de $\mathscr{M}^{\otimes n}$ vers $\mathscr{L}$ définit une section $s: X \rightarrow\left[\mathscr{L}^{\frac{1}{n}}\right]$ du morphisme structural $\pi$. Donc la gerbe fppf $\left[\mathscr{L}^{\frac{1}{n}}\right]$ sur $X$ est une gerbe neutre (au sens de [34] (3.20)). Le résultat découle donc de l'analogue fppf de [34] (3.21).

3 ) résulte de 2) et du fait que $\mathscr{L}$ est localement trivial pour la topologie de Zariski.

Remarque 5.3.2 Si $n$ est inversible, alors $\mu_{n}$ est étale et les champs $\left(\mathrm{B} \mu_{n}\right)_{\mathrm{fppf}}$ et $\mathrm{B} \mu_{n}$ coïncident ([34] (9.6)). Dans ce cas ce sont des champs de Deligne-Mumford. Notons que si $n$ n'est pas inversible, alors $\mu_{n}$ n'est pas lisse, et le champ B $\mu_{n}$ qui classifie les $\mu_{n}$-torseurs étales n'a aucune raison a priori d'être algébrique.

Remarque 5.3.3 On suppose que $S$ est un $\mathbb{Z}\left[\frac{1}{n}\right]$-schéma et que $X$ est noethérien. Alors $\pi$ est propre, lisse, de présentation finie, et cohomologiquement plat en dimension zéro. En particulier si $X / S$ vérifie ces propriétés, le morphisme $\left[\mathscr{L}^{\frac{1}{n}}\right] \rightarrow S$ les vérifie aussi.

CAlCUl DU GROUPE DE PICARD DE $\left[\mathscr{L}^{\frac{1}{n}}\right]$

Lemme 5.3.4 Soient $X$ un schéma et A un schéma en groupes commutatifs sur X. Soit $\mathscr{F}$ un faisceau inversible sur une A-gerbe (fppf) $\pi: \mathscr{X} \rightarrow X$. Il existe un unique $X$-morphisme de schémas en groupes

$$
\chi \mathscr{F}: A \longrightarrow \mathbb{G}_{\mathrm{m}}
$$

tel que l'action naturelle de A sur $\mathscr{F}$ soit induite par $\chi_{\mathscr{F}}$ et par la multiplication $\mathscr{F} \times \mathbb{G}_{\mathrm{m}} \rightarrow \mathscr{F}$ induite par la structure de $\mathscr{O}_{\mathscr{X}}$-module de $\mathscr{F}$, autrement dit tel que le diagramme suivant soit commutatif:

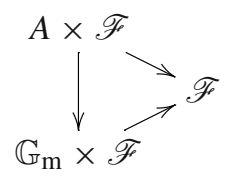

Démonstration. Un faisceau inversible sur la gerbe $\mathscr{X}$ est la donnée, pour tout $x \in$ ob $\mathscr{X}_{U}$, d'un faisceau inversible $\mathscr{F}_{x}$ sur $U$, et pour tout morphisme $\varphi: x \rightarrow x^{\prime}$ dans $\mathscr{X}$, d'un isomorphisme

$$
L_{\mathscr{F}}(\varphi): \mathscr{F}_{x} \rightarrow \pi(\varphi)^{*} \mathscr{F}_{x^{\prime}}
$$

ces isomorphismes vérifiant de plus une condition de compatibilité évidente.

Construisons d'abord $\chi \mathscr{F}(U)$ pour un $U \in$ ob (Aff $/ X)$ sur lequel $\mathscr{X}$ a des objets. Soit $x \in$ ob $\mathscr{X}_{U}$ et soit $g \in A(U)$. Via l'identification entre $A(U)$ et Aut $(x), g$ correspond à un automorphisme $\varphi$ de $x$, et induit de ce fait un automorphisme $L_{\mathscr{F}}(\varphi)$ de $\mathscr{F}_{x}$. 
Cet automorphisme correspond à la multiplication par un unique élément de $\mathbb{G}_{\mathrm{m}}(U)$, dont on vérifie facilement qu'il ne dépend pas du choix de $x$ (en utilisant la condition de compatibilité entre les $L_{\mathscr{F}}(\varphi)$ et le fait que deux objets de $\mathscr{X}_{U}$ sont localement isomorphes pour la topologie $f p p f)$. D'où le morphisme $\chi_{\mathscr{F}}(U): A(U) \rightarrow \mathbb{G}_{\mathrm{m}}(U)$. Il est clair, vu sa construction, que ce morphisme est déterminé de manière unique par les actions naturelles de $A$ et de $\mathbb{G}_{\mathrm{m}}$ sur $\mathscr{F}$. Étant donné que $\mathscr{X}$ a des objets partout localement pour la topologie fppf, cette collection de morphismes se prolonge de manière unique en un caractère $\chi \mathscr{F}$ de $A$ vérifiant les propriétés annoncées.

Remarque 5.3.5 Si $\chi$ est un caractère fixé de $A$, un faisceau inversible $\mathscr{F}$ sur $\mathscr{X}$ est un faisceau $\chi$-tordu de degré $d$ (au sens de Lieblich [35] 2.1.2.2) si et seulement si $\chi \mathscr{F}=\chi^{d}$.

Propriétés 5.3.6 (1) La construction de $\chi_{\mathscr{F}}$ est compatible au changement de base.

(2) Si $\mathscr{F}$ et $\mathscr{G}$ sont des faisceaux inversibles sur $\mathscr{X}$, alors

$$
\chi \mathscr{F} \otimes \mathscr{G}=\chi \mathscr{F} \cdot \chi_{\mathscr{G}} .
$$

(3) Un faisceau inversible $\mathscr{F}$ sur $\mathscr{X}$ provient de $X$ si et seulement si $\chi \mathscr{F}$ est trivial.

Démonstration. Les deux premières propriétés sont évidentes. Montrons le dernier point. Supposons tout d'abord que $\mathscr{F}$ soit isomorphe à un faisceau de la forme $\pi^{*} \mathscr{M}$, où $\mathscr{M}$ est un faisceau inversible sur $X$. Il est clair que $\chi_{\mathscr{F}}$ est égal à $\chi_{\pi^{*} \mathscr{M}}$ donc il suffit de montrer que $\chi_{\pi * \mathscr{M}}$ est trivial, c'est-à-dire que pour tout objet $x$ de $\mathscr{X}$ et pour tout automorphisme $\varphi$ de $x$, l'automorphisme $L_{\pi^{*} \mathscr{M}}(\varphi)$ de $\left(\pi^{*} \mathscr{M}\right)_{x}$ est l'identité. C'est évident par construction de l'image inverse.

Réciproquement, supposons $\chi \mathscr{F}$ trivial, i.e. supposons que pour tout objet $x$ de $\mathscr{X}$ et tout automorphisme $\varphi$ de $x, L_{\mathscr{F}}(\varphi)$ soit l'identité de $\mathscr{F}_{x}$. Provenir de la base est une question locale sur $X$ pour la topologie fppf (voir par exemple le lemme 1.2.2.7 de [15]). On peut donc supposer que le morphisme structural $\pi: \mathscr{X} \rightarrow X$ a une section $s: X \rightarrow \mathscr{X}$.

On va alors montrer que $\mathscr{F}$ est isomorphe à $\pi^{*} s^{*} \mathscr{F}$. Le faisceau $\pi^{*} s^{*} \mathscr{F}$ est celui qui à tout objet $x$ de $\mathscr{X}$ associe $\mathscr{F}_{s(\pi(x))}$, les isomorphismes de changement de base étant simplement les isomorphismes canoniques. Nous allons construire une collection d'isomorphismes $\rho_{x}$ de $\mathscr{F}_{x}$ dans $\mathscr{F}_{S(\pi(x))}$ (pour chaque objet $x$ de $\mathscr{X}$ ), compatibles avec les $L_{\mathscr{F}}(\varphi)$ et les $L_{\pi *} s^{*} \mathscr{F}(\varphi)$.

Si $x$ et $s(\pi(x))$ sont isomorphes dans $\mathscr{X}_{U}$ on choisit un isomorphisme $\varphi$ de $x$ dans $s(\pi(x))$ et on pose $\rho_{x}=L_{\mathscr{F}}(\varphi)$. Si $\varphi_{1}$ et $\varphi_{2}$ sont deux tels isomorphismes, alors $\left(\varphi_{2}\right)^{-1} \circ \varphi_{1}$ est un automorphisme de $x$, donc d'après l'hypothèse sur $\mathscr{F}$ on a $L \mathscr{F}\left(\left(\varphi_{2}\right)^{-1} \circ \varphi_{1}\right)=\operatorname{Id} \mathscr{F}_{x}$ de sorte que $\rho_{x}$ est bien défini et ne dépend pas du choix de $\varphi$.

Dans le cas général, on sait que $x$ et $s(\pi(x))$ sont localement isomorphes pour la topologie fppf. Vu l'unicité dans la construction de $\rho_{x}$ lorsque $x$ est isomorphe à $s(\pi(x))$, il est clair qu'il existe un unique isomorphisme $\rho_{x}: \mathscr{F}_{x} \rightarrow \mathscr{F}_{s}(\pi(x))$ compatible avec ceux construits dans le cas précédent. La collection de tous les $\rho_{x}$ ainsi construits répond au problème posé. 
Exemple 5.3.7 (groupe de Picard de $\mathrm{B} G$ ) En utilisant cette construction, on retrouve facilement le groupe de Picard du champ classifiant $\mathrm{B} G$, où $G$ est un $X$-schéma en groupes abéliens. En effet, le morphisme structural $\pi: \mathrm{B} G \rightarrow X$ a une section, donc $\pi^{*}: \operatorname{Pic}(X) \rightarrow \operatorname{Pic}(\mathrm{B} G)$ a une rétraction et en particulier il est injectif. D'après 5.3.6, l'application $\mathscr{F} \mapsto \chi \mathscr{F}$ induit un morphisme de groupes de $\operatorname{Pic}(\mathrm{B} G)$ dans $\widehat{G}$ dont $\operatorname{Pic}(X)$ est le noyau. Ce morphisme est naturellement scindé : si $\chi: G \rightarrow \mathbb{G}_{\mathrm{m}}$ est un caractère de $G$, on lui associe la classe du faisceau inversible $\mathscr{L}(\chi)$ construit de la manière suivante. Pour tout $U \in$ ob (Aff/S) et tout $G$-torseur $\widetilde{U}$ on définit $\mathscr{L}(\chi)_{\widetilde{U}}$ comme étant le faisceau inversible correspondant au $\mathbb{G}_{\mathrm{m}}$-torseur sur $U$ obtenu à partir de $\widetilde{U}$ par extension du groupe structural via le caractère $\chi$. On a donc une suite exacte courte scindée:

$$
1 \longrightarrow \operatorname{Pic}(X) \longrightarrow \operatorname{Pic}(\mathrm{B} G) \longrightarrow \widehat{G} \longrightarrow 1
$$

de sorte que $\operatorname{Pic}(\mathrm{B} G)$ est naturellement isomorphe au produit $\operatorname{Pic}(X) \times \widehat{G}$.

Dans le cas du champ $\left[\mathscr{L}^{\frac{1}{n}}\right]$, on a un faisceau inversible «canonique », que nous noterons $\Omega$, défini par $\Omega_{\alpha}=\mathscr{M}$ pour tout $U \in$ ob (Aff/S) et tout objet $\alpha=(x, \mathscr{M}, \varphi)$ de $\left[\mathscr{L}^{\frac{1}{n}}\right]_{U}$. Il est clair que le caractère $\chi_{\Omega}$ associé à $\Omega$ est simplement l'injection canonique

$$
\chi: \mu_{n} \longrightarrow \mathbb{G}_{\mathrm{m}} .
$$

En particulier, vu que $\chi$ n'est pas le caractère trivial, on peut en déduire que le faisceau $\Omega$ ne provient pas de la base $X$ ! On a enfin un isomorphisme canonique $\Phi: \Omega^{\otimes n} \stackrel{\sim}{\longrightarrow} \pi^{*} \mathscr{L}$.

Proposition 5.3.8 On note l la classe du faisceau $\mathscr{L}$ dans $\operatorname{Pic}(X)$ et $\omega$ celle de $\Omega$ dans $\operatorname{Pic}\left(\left[\mathscr{L}^{\frac{1}{n}}\right]\right)$.

(1) Le morphisme $\pi^{*}: \operatorname{Pic}(X) \rightarrow \operatorname{Pic}\left(\left[\mathscr{L}^{\frac{1}{n}}\right]\right)$ est injectif, et l'on a une suite exacte courte:

$$
1 \longrightarrow \operatorname{Pic}(X) \longrightarrow \operatorname{Pic}\left(\left[\mathscr{L}^{\frac{1}{n}}\right]\right) \longrightarrow \widehat{\mu_{n}} \longrightarrow 1
$$

(2) Le groupe $\operatorname{Pic}\left(\left[\mathscr{L}^{\frac{1}{n}}\right]\right)$ est isomorphe au quotient du groupe $\operatorname{Pic}(X) \times H^{0}(X, \mathbb{Z})$ parle sous $H^{0}(X, \mathbb{Z})$-module engendré par $\left(l^{-1}, n\right)$ (autrement dit par la relation $\left.\omega^{n}=l\right)$.

Démonstration. La propriété 5.3.6 (2) montre que l'application $\mathscr{F} \mapsto \chi \mathscr{F}$ induit un morphisme de groupes de $\operatorname{Pic}\left(\left[\mathscr{L}^{\frac{1}{n}}\right]\right)$ dans $\widehat{\mu_{n}}$. Il est clair que ce morphisme est surjectif, vu que $\widehat{\mu_{n}}$ est isomorphe au groupe $H^{0}(X, \mathbb{Z} / n \mathbb{Z})$, engendré par l'injection canonique $\chi: \mu_{n} \rightarrow \mathbb{G}_{\mathrm{m}}$, et que $\chi=\chi_{\Omega}$. La propriété 5.3.6 (3) montre que la suite ci-dessus est exacte en $\operatorname{Pic}\left(\left[\mathscr{L}^{\frac{1}{n}}\right]\right)$. Pour en finir avec le premier point il nous reste donc juste à montrer l'injectivité de $\pi^{*}$. 
Soit $\mathscr{N}$ un faisceau inversible sur $X$ et soit $f$ un isomorphisme de $\pi^{*} \mathscr{N}$ dans $\mathscr{O}_{\left[\mathscr{L}^{\frac{1}{n}}\right]}$. Il s'agit de montrer que $\mathscr{N}$ est trivial. L'isomorphisme $f$ est donné par une collection d'isomorphismes

$$
\left(f_{\alpha}:\left(\pi^{*} \mathscr{N}\right)_{\alpha}=x^{*} \mathscr{N} \stackrel{\sim}{\longrightarrow}\left(\mathscr{O}_{\left[\mathscr{L}^{\frac{1}{n}}\right]}\right)_{\alpha}=\mathscr{O}_{U}\right)_{\alpha=(x, \mathscr{M}, \varphi) \in\left[\mathscr{L}^{\frac{1}{n}}\right]_{U}} .
$$

En utilisant la condition de compatibilité vérifiée par les $f_{\alpha}$ et la structure de gerbe de $\left[\mathscr{L}^{\frac{1}{n}}\right]$, on construit une famille compatible d'isomorphismes $f_{x}: x^{*} \mathscr{N} \stackrel{\sim}{\longrightarrow} \mathscr{O}_{U}$ indexée par les $x \in X(U), U \in \mathrm{ob}$ (Aff/S), qui définit donc un isomorphisme de $\mathscr{N}$ dans $\mathscr{O}_{X}$.

Pour le point (2), notons $G$ le quotient du groupe $\operatorname{Pic}(X) \times H^{0}(X, \mathbb{Z})$ par le sous $H^{0}(X, \mathbb{Z})$-module engendré par la relation $\omega^{n}=l$. On a clairement un morphisme de $G$ dans $\operatorname{Pic}\left(\left[\mathscr{L}^{\frac{1}{n}}\right]\right)$ qui envoie $(0,1)$ sur $\omega$. En utilisant les propriétés précédentes, et le fait que $\widehat{\mu_{n}}$ est isomorphe à $H^{0}(X, \mathbb{Z} / n \mathbb{Z})$ et engendré par $\chi_{\Omega}$, on vérifie facilement que ce morphisme est un isomorphisme.

Exemple 5.3.9 Prenons pour $X$ l'espace projectif $\mathbb{P}^{k}$ sur $\operatorname{Spec} \mathbb{Z}$. On fixe un entier relatif $l$ et on pose $\mathscr{L}=\mathscr{O}(l)$. La proposition précédente permet de calculer $\operatorname{Pic}\left(\left[\mathscr{L}^{\frac{1}{n}}\right]\right)$ pour tout $n$ appartenant à $\mathbb{N}^{*}$. Par exemple si $l=1$, on trouve $\frac{1}{n} \mathbb{Z}$. Si $l$ est un multiple de $n$, on est dans le cas où $\mathscr{L}$ a une racine $n^{\text {ième }}$ et l'on trouve $\mathbb{Z} \times \mathbb{Z} / n \mathbb{Z}$. Dans le cas général, le groupe $\operatorname{Pic}\left(\left[\mathscr{L}^{\frac{1}{n}}\right]\right)$ est isomorphe (de manière non canonique) à $\frac{d}{n} \mathbb{Z} \times \mathbb{Z} / d \mathbb{Z}$ où $d$ est le pgcd de $n$ et $l$.

FONCTEUR DE PICARD RELATIF DE $\left[\mathscr{L}^{\frac{1}{n}}\right] / S$

Notons $\mathscr{X}=\left[\mathscr{L}^{\frac{1}{n}}\right]$. Pour tout schéma $U$ sur $S$, on a une suite exacte courte:

$$
1 \longrightarrow \operatorname{Pic}\left(X \times{ }_{S} U\right) \longrightarrow \operatorname{Pic}\left(\mathscr{X} \times{ }_{S} U\right) \longrightarrow H^{0}\left(X \times{ }_{S} U, \mathbb{Z} / n \mathbb{Z}\right) \longrightarrow 1 \text {. }
$$

En appliquant le foncteur «faisceau étale associé » elle induit une suite exacte de faisceaux étales:

$$
1 \longrightarrow \operatorname{Pic}_{X / S} \stackrel{\varphi_{0}}{\longrightarrow} \operatorname{Pic}_{\mathscr{X} / S} \stackrel{\chi}{\longrightarrow} f_{*} \mathbb{Z} / n \mathbb{Z} \longrightarrow 1,
$$

où $f$ désigne le morphisme structural de $X$ dans $S$.

Remarque 5.3.10 $\mathrm{Si} \mathscr{L}$ a une racine $n^{\text {ième }} \mathscr{R}$, la suite exacte (2) est scindée par $i \mapsto$ $\left(\omega r^{-1}\right)^{i}$ où $r$ est la classe de $\mathscr{R}$ dans $\operatorname{Pic}(X)$, si bien que $\operatorname{Pic} \mathscr{X} / S$ s'identifie au produit $\operatorname{Pic}_{X / S} \times_{S} f_{*} \mathbb{Z} / n \mathbb{Z}$.

Remarque 5.3.11 Le faisceau $f_{*} \mathbb{Z} / n \mathbb{Z}$ n'est a priori pas représentable. En conséquence, dans le cas général, il ne suffit pas que $\mathrm{Pic}_{X / S}$ soit représentable pour que Pic $\mathscr{X} / S$ le soit, même lorsque la gerbe $\mathscr{X}$ est triviale. Cependant si $f$ est universellement ouvert, surjectif et à fibres géométriquement connexes (par exemple s'il est localement de type fini et cohomologiquement plat), alors $f_{*} \mathbb{Z} / n \mathbb{Z}=\mathbb{Z} / n \mathbb{Z}$. 
On suppose maintenant que $f_{*} \mathbb{Z} / n \mathbb{Z}$ coïncide avec $\mathbb{Z} / n \mathbb{Z}$ et l'on considère le produit $\operatorname{Pic}_{X / S} \times_{S} \mathbb{Z}$ de $\operatorname{Pic}_{X / S}$ par le groupe constant $\mathbb{Z}$. On va voir que Pic $\mathscr{X} / S$ est isomorphe au quotient de $\operatorname{Pic}_{X / S} \times{ }_{S} \mathbb{Z}$ par la relation $\omega^{n}=l$. On note $H$ le sousgroupe engendré par $\left(l^{-1}, n\right)$. En notant $\varphi_{i}$ le morphisme composé $\operatorname{Pic}_{X / S} \stackrel{\varphi_{0} \longrightarrow}{\longrightarrow}$ $\operatorname{Pic} \mathscr{X} / S \stackrel{\mu_{\omega^{i}}}{\longrightarrow} \operatorname{Pic} \mathscr{X}_{/ S}$ (où $\mu_{\omega^{i}}$ est la multiplication par $\omega^{i}$ ) on définit donc un morphisme

$$
\varphi: \operatorname{Pic}_{X / S} \times{ }_{S} \mathbb{Z} \longrightarrow \operatorname{Pic} \mathscr{X} / S
$$

dont il est clair qu'il est invariant sous $H$ et universel pour cette propriété. Le foncteur Pic $\mathscr{X} / S$ s'identifie donc bien au quotient évoqué ci-dessus. On peut construire ce quotient «à la main » comme suit (voir figure ci-dessous). Pour tout couple d'entiers $(i, k)$ on identifie les copies de $\operatorname{Pic}_{X / S}$ numéro $i$ et $i+n k$ via l'isomorphisme de translation $\mu_{l^{k}}:\left(\operatorname{Pic}_{X / S}\right)_{i+n k} \rightarrow\left(\operatorname{Pic}_{X / S}\right)_{i}$. La loi de groupe est induite naturellement par celle de $\operatorname{Pic}_{X / S}$ et par la relation $\omega^{n}=l$. Ceci montre en particulier que si $\mathrm{Pic}_{X / S}$ est représentable, alors Pic $\mathscr{X} / S$ l'est aussi. ${ }^{7}$ On en déduit aussi que $\mathrm{Pic}_{X / S}$ et $\mathrm{Pic}_{\mathscr{X}} / S$ ont la même composante neutre.

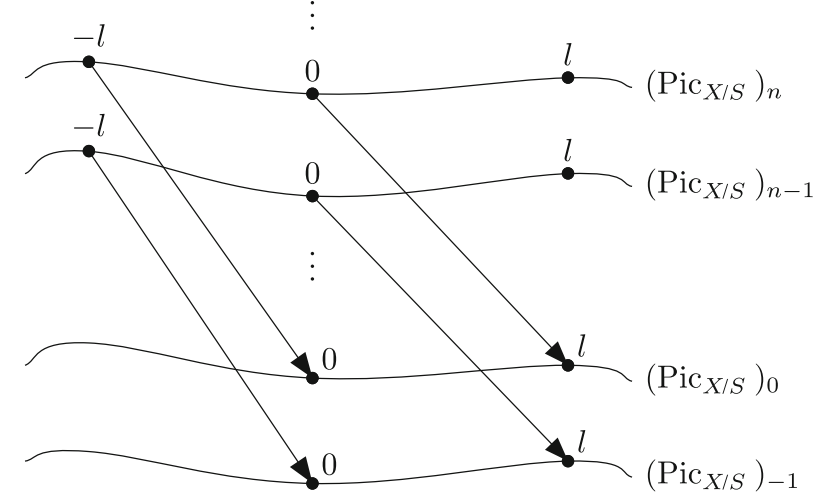

Le foncteur de Picard de $\left[\mathscr{L}^{\frac{1}{n}}\right]$

DESCRIPTION DU CHAMP DE PICARD DE $\left[\mathscr{L}^{\frac{1}{n}}\right]$

On a une «suite exacte» de champs de Picard:

$$
1 \longrightarrow \operatorname{PP} i c(X / S) \stackrel{\pi^{*}}{\longrightarrow} \operatorname{P} i c(\mathscr{X} / S) \stackrel{\chi}{\longrightarrow} f_{*} \mathbb{Z} / n \mathbb{Z} \longrightarrow 1 \text {. }
$$

\footnotetext{
7 Mais bien sûr, on le savait déjà dans le cas où $f$ est propre, plat et cohomologiquement plat en dimension zéro.
} 
Autrement dit, $\pi^{*}$ est pleinement fidèle, $\chi$ est un épimorphisme, et si $\mathscr{F}$ est un objet de $\mathscr{P} i c(\mathscr{X} / S)$, il provient de $\mathscr{P} i c(X / S)$ si et seulement si son caractère $\chi_{\mathscr{F}}$ est nul. Tout ceci a déjà été prouvé. De même que précédemment, si l'on suppose que $f_{*} \mathbb{Z} / n \mathbb{Z}=\mathbb{Z} / n \mathbb{Z}$, alors le champ $\mathscr{P} i c(\mathscr{X} / S)$ s'identifie au champ obtenu à partir de $\mathscr{P} i c(X / S) \times{ }_{S} \mathbb{Z}$ en recollant les copies numéro $i$ et $i+n k$ le long de l'isomorphisme $\mu_{l^{k}}:\left(\mathscr{P}_{i c}(X / S)\right)_{i+n k} \longrightarrow\left(\mathscr{P}_{i c}(X / S)\right)_{i}$ pour tous $i, k$ appartenant à $\mathbb{Z}$. En particulier il suffit dans ce cas que $\mathscr{P} i c(X / S)$ soit algébrique pour que $\mathscr{P} i c(\mathscr{X} / S)$ le soit aussi. Dans le cas où $\mathscr{L}$ a une racine $n^{\text {ième } \mathscr{R}}$ sur $X$, la suite exacte (3) est scindée et $\mathscr{P}$ ic $(\mathscr{X} / S)$ s'identifie au produit $\mathscr{P}$ ic $(X / S) \times{ }_{S} f_{*} \mathbb{Z} / n \mathbb{Z}$.

\subsection{Courbes tordues d'Abramovich et Vistoli}

Abramovich et Vistoli ont mis au jour dans [6-8] une classe de courbes « tordues » qui apparaissent naturellement lorsque l'on cherche à compactifier certains espaces de modules. Ces courbes sont des courbes nodales munies d'une «structure champêtre » supplémentaire aux points singuliers ou en certains points marqués. Nous nous proposons de décrire le foncteur de Picard des courbes tordues lisses. Nous allons voir que la structure supplémentaire modifie le foncteur de Picard de la courbe d'une manière très analogue à ce que nous avons pu observer dans la section précédente. Nous commençons par quelques rappels sur les courbes tordues.

Définition 5.4.1 ([8, 4.1.2] ou [43, 1.2]) Soit $S$ un schéma. Une courbe tordue sur $S$ est un champ de Deligne-Mumford $f: \mathscr{C} \rightarrow S$ modéré, ${ }^{8}$ propre, plat et de présentation finie sur $S$ dont les fibres sont purement de dimension 1, géométriquement connexes et ont au plus des singularités nodales, vérifiant de plus les propriétés suivantes:

1) $\mathrm{Si} \pi: \mathscr{C} \rightarrow C$ est l'espace de modules grossier de $\mathscr{C}$ et si $C_{\text {lis }}$ est le lieu lisse de $C$ sur $S$, alors le sous-champ ouvert $\mathscr{C} \times{ }_{C} C_{\text {lis }}$ est le lieu lisse de $\mathscr{C}$ sur $S$.

2) Pour tout point géométrique $\bar{s} \rightarrow S$ le morphisme induit $\mathscr{C}_{\bar{S}} \rightarrow C_{\bar{s}}$ est un isomorphisme au-dessus d'un ouvert dense de $C_{\bar{s}}$.

Une courbe tordue $n$-pointée est une courbe tordue munie d'une collection $\left\{\Sigma_{i}\right\}_{i=1}^{n}$ de sous-champs fermés de $\mathscr{C}$ deux à deux disjoints tels que:

(i) Pour tout $i$, le sous-champ fermé $\Sigma_{i}$ est dans le lieu lisse de $\mathscr{C}$.

(ii) Pour tout $i$, le morphisme $\Sigma_{i} \rightarrow \mathscr{C} \rightarrow S$ est une gerbe étale sur $S$.

(iii) Si $\mathscr{C}_{\text {gén }}$ est l'ouvert complémentaire des $\Sigma_{i}$ dans $\mathscr{C}_{\text {lis }}$, alors $\mathscr{C}_{\text {gén }}$ est un schéma.

Remarque 5.4.2 D'après la proposition 4.1.1 de [8], l'espace de modules grossier $C$ est une courbe nodale propre et plate sur $S$, de présentation finie et à fibres géométriquement connexes. Si de plus la courbe $\mathscr{C}$ est $n$-pointée, alors l'espace de modules grossier $D_{i}$ du sous-champ fermé $\Sigma_{i}$ est naturellement un sous-schéma fermé de $C$, et le morphisme composé $D_{i} \rightarrow C \rightarrow S$ est un isomorphisme. Les $D_{i}$ définissent donc des sections de $C \rightarrow S$ qui en font une courbe nodale $n$-pointée au sens usuel.

\footnotetext{
${ }^{8}$ Un champ algébrique $\mathscr{C}$ est dit modéré si pour tout corps algébriquement clos $k$ et tout morphisme $x: \operatorname{Spec} k \rightarrow \mathscr{C}$, le groupe $\operatorname{Aut}_{\mathscr{C}}(x)$ est d'ordre inversible dans $k$.
} 
Théorème 5.4.3 ([8, 3.2.3] ou [43, 2.2]) Au voisinage (étale) d'un point marqué, la courbe $\mathscr{C} \rightarrow S$ est de la forme $\left[U / \mu_{r}\right] \rightarrow \operatorname{Spec} A$ où $U=\operatorname{Spec} A[x]$ et où un générateur de $\mu_{r}$ agit sur $U$ par $x \mapsto \xi$.x avec $\xi$ une racine primitive $r^{\text {ième }}$ de l'unité.

Au voisinage d'un noud, la courbe $\mathscr{C} \rightarrow S$ est de la forme $\left[U / \mu_{r}\right] \rightarrow \operatorname{Spec} A$ où $U=\operatorname{Spec}(A[x, y] /(x y-t))$ pour un certain $t \in A$ et où un générateur de $\mu_{r}$ agit sur $U$ par $(x, y) \mapsto\left(\xi . x, \xi^{\prime} \cdot y\right)$ avec $\xi$ et $\xi^{\prime}$ des racines primitives rèmes de l'unité.

Remarque 5.4.4 Si $p \in C$ est un nœud fixé, et si $\xi$ et $\xi^{\prime}$ sont les racines primitives $r^{\text {ièmes }}$ de l'unité qui apparaissent ci-dessus, on dit que le nœud $p$ est «balancé » si le produit $\xi . \xi^{\prime}$ est égal à 1 . On dit que la courbe $\mathscr{C}$ est balancée si tous ses nœuds sont balancés. Signalons que si $p$ n'est pas balancé, on a nécessairement $t=0$ dans la description locale ci-dessus. Autrement dit on ne peut pas faire disparaître le nœud en déformant la courbe $([43,2.2])$.

Signalons qu'Olsson montre dans [43] que se donner une courbe tordue revient à se donner une courbe nodale classique munie d'une certaine «structure logarithmique». Pour les courbes tordues lisses, Cadman donne une autre description, plus élémentaire.

Théorème 5.4.5 ([16, 2.2.4 et 4.1]) Se donner une courbe tordue $\left(\mathscr{C},\left\{\Sigma_{i}\right\}_{i=1}^{n}\right)$ $n$-pointée lisse sur un schéma $S$ noethérien et connexe est équivalent à se donner une courbe n-pointée $\left(C,\left\{\sigma_{i}\right\}_{i=1}^{n}\right)$ lisse sur $S$ et un $n$-uplet $\vec{r}=\left(r_{1}, \ldots, r_{n}\right)$ d'entiers strictement positifs inversibles sur $S$. La courbe tordue $\mathscr{C}$ est alors isomorphe au champ $C_{\mathbb{D}, \vec{r}}$ défini de la manière suivante.

Chaque section $\sigma_{i}$ définit un diviseur de Cartier effectif $D_{i}$ de $C$. On note $s_{D_{i}}$ la section canonique de $\mathscr{O}\left(D_{i}\right)$ qui s'annule sur $D_{i}$. La collection des $\mathscr{O}\left(D_{i}\right)$ et des $s_{D_{i}}$ correspond à un morphisme $C \rightarrow\left[\mathbb{A}^{n} / \mathbb{G}_{\mathrm{m}}^{n}\right]$. On note aussi $\theta_{\vec{r}}$ le morphisme de $\left[\mathbb{A}^{n} / \mathbb{G}_{\mathrm{m}}^{n}\right]$ dans lui-même qui envoie un $n$-uplet de faisceaux inversibles $\left(L_{1}, \ldots, L_{n}\right)$ muni de sections $\left(t_{1}, \ldots, t_{n}\right)$ sur le $n$-uplet $\left(L_{1}^{r_{1}}, \ldots, L_{n}^{r_{n}}\right)$ muni de $\left(t_{1}^{r_{1}}, \ldots, t_{n}^{r_{n}}\right)$. On définit alors $C_{\mathbb{D}, \vec{r}}$ comme étant le produit fibré

$$
C \times\left[\mathbb{A}^{n} / \mathbb{G}_{\mathrm{m}}^{n}\right], \theta_{\vec{r}}\left[\mathbb{A}^{n} / \mathbb{G}_{\mathrm{m}}^{n}\right]
$$

Cadman décrit dans [16] les faisceaux inversibles sur une courbe tordue lisse sur une base connexe et noethérienne (corollaire 3.2.1) : un faisceau inversible sur $C_{\mathbb{D}, \vec{r}}$ s'écrit de manière unique sous la forme $\pi^{*} L \otimes \prod_{i=1}^{n} \mathscr{T}_{i}^{\otimes k_{i}}$ où $\pi: \mathscr{C} \rightarrow C$ est la projection de $\mathscr{C}$ sur son espace de modules grossier, $L$ est un faisceau inversible sur $C, \mathscr{T}_{i}$ est le faisceau inversible $\mathscr{O}_{\mathscr{C}}\left(\Sigma_{i}\right)$ et $k_{i}$ est un entier compris entre 0 et $r_{i}-1$. Il est clair que les hypothèses noethériennes ne sont pas essentielles pour ce résultat. Par ailleurs on peut aussi supprimer l'hypothèse de connexité sur la base : il faut alors remplacer les entiers $k_{i}$ par des fonctions localement constantes à valeurs dans $\mathbb{Z}$. On obtient ainsi le théorème suivant.

Théorème 5.4.6 [16, corollaire 3.2.1] Soient $S$ un schéma, $C$ une courbe lisse n-pointée sur $S, \vec{r}$ un n-uplet d'entiers strictement positifs et $C_{\mathbb{D}, \vec{r}}$ la courbe tordue associée par la construction de Cadman. Soit $\mathscr{L}$ un faisceau inversible sur $C_{\mathbb{D}, \vec{r}}$. Alors il existe un faisceau inversible $L$ sur $C$ et des fonctions localement constantes $k_{i}$ appartenant à $H^{0}(S, \mathbb{Z})$ prenant leurs valeurs dans $\left\{0, \ldots, r_{i}-1\right\}$ tels que 


$$
\mathscr{L} \simeq \pi^{*} L \otimes \prod_{i=1}^{n} \mathscr{T}_{i}^{k_{i}}
$$

De plus les fonctions $k_{i}$ sont uniques, L est unique à isomorphisme près, et $\mathscr{T}_{i}^{r_{i}}$ est isomorphe à $\pi^{*} \mathscr{O}\left(D_{i}\right)$.

En particulier on a une suite exacte courte:

$$
0 \longrightarrow \operatorname{Pic}(C) \longrightarrow \operatorname{Pic}(\mathscr{C}) \longrightarrow \prod_{i=1}^{n} H^{0}\left(S, \mathbb{Z} / r_{i} \mathbb{Z}\right) \longrightarrow 0
$$

Signalons que si $S$ est le spectre d'un corps algébriquement clos, Chiodo ([17]) obtient cette suite exacte d'une autre manière pour une courbe tordue quelconque.

Soit $\left(\mathscr{C},\left\{\Sigma_{i}\right\}_{i=1}^{n}\right)$ une courbe tordue $n$-pointée lisse sur une base $S$ noethérienne et connexe. D'après le théorème $5.4 .5, \mathscr{C}$ est isomorphe au champ $C_{\mathbb{D}, \vec{r}}$ où $C$ est l'espace de modules grossier de $\mathscr{C}, \vec{r}$ est un $n$-uplet d'entiers positifs et $\mathbb{D}=\left(D_{1}, \ldots, D_{n}\right)$ est le $n$-uplet de diviseurs effectifs de Cartier de $C$ correspondant aux $\Sigma_{i}$. Pour tout $i$ le morphisme de $D_{i}$ vers $S$ est un isomorphisme. On note $\mathscr{T}_{i}$ le faisceau $\mathscr{O}_{\mathscr{C}}\left(\Sigma_{i}\right)$. Alors toutes ces données sont compatibles au changement de base. Plus précisément, si $T \rightarrow S$ est un morphisme de changement de base, le produit fibré $\mathscr{C} \times{ }_{S} T$ est isomorphe au champ $C_{\mathbb{D}^{\prime}, r}^{\prime}$ où $C^{\prime}\left(\right.$ resp. $D_{i}^{\prime}$ ) est le produit fibré $C \times{ }_{S} T$ (resp. $D_{i} \times{ }_{S} T$ ) et $\mathbb{D}^{\prime}=\left(D_{1}^{\prime}, \ldots, D_{n}^{\prime}\right)$. De plus le faisceau inversible canonique $\mathscr{T}_{i}^{\prime}$ n'est autre que $\Phi^{*} \mathscr{T}_{i}$ où $\Phi$ est la projection de $\mathscr{C}^{\prime}$ sur $\mathscr{C}$. En appliquant le théorème précédent à la courbe $\mathscr{C}^{\prime}$, on obtient pour tout $T$ une suite exacte courte

$$
0 \longrightarrow \operatorname{Pic}\left(C \times{ }_{S} T\right) \longrightarrow \operatorname{Pic}\left(\mathscr{C} \times{ }_{S} T\right) \longrightarrow \prod_{i=1}^{n} H^{0}\left(T, \mathbb{Z} / r_{i} \mathbb{Z}\right) \longrightarrow 0
$$

En appliquant le foncteur «faisceau étale associé » elle induit une suite exacte courte de faisceaux étales:

$$
0 \longrightarrow \operatorname{Pic}_{C / S} \longrightarrow \operatorname{Pic}_{\mathscr{C} / S} \longrightarrow \prod_{i=1}^{n} \mathbb{Z} / r_{i} \mathbb{Z} \longrightarrow 0
$$

Notons $l_{i}$ la classe de $\mathscr{O}_{C}\left(D_{i}\right)$ dans $\operatorname{Pic}_{C / S}(S)$ et $t_{i}$ la classe de $\mathscr{T}_{i}$ dans $\operatorname{Pic}_{\mathscr{C} / S}(S)$. On a dans $\operatorname{Pic}_{\mathscr{C} / S}$ la relation $t_{i}^{r_{i}}=l_{i}$. En procédant comme pour le cas du champ

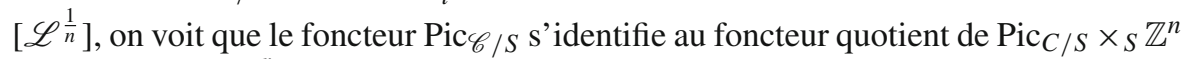
par les relations $t_{i}^{r_{i}}=l_{i}$ (où par abus les $t_{1}, \ldots, t_{n}$ désignent aussi les générateurs canoniques de $\mathbb{Z}^{n}$ ). On peut construire ce quotient à la main comme suit. Le produit $\operatorname{Pic}_{C / S} \times{ }_{S} \mathbb{Z}^{n}$ est une union disjointe de copies de Pic ${ }_{C / S}$ indexées par les $n$-uplets $\underline{\alpha}=\left(\alpha_{1}, \ldots, \alpha_{n}\right)$ appartenant à $\mathbb{Z}^{n}$. Alors $\operatorname{Pic}_{\mathscr{C} / S}$ est obtenu en identifiant pour tout $\underline{\alpha}$, pour tout entier $k$ appartenant à $\mathbb{Z}$ et pour tout entier $i$ compris entre 1 et $n$, les copies 
$\left(\operatorname{Pic}_{C / S}\right)_{\underline{\alpha}}$ et $\left(\operatorname{Pic}_{C / S}\right)_{\left(\alpha_{1}, \ldots, \alpha_{i}+k r_{i}, \ldots, \alpha_{n}\right)}$ via l'isomorphisme de multiplication par $l_{i}^{k}$ de $\left(\operatorname{Pic}_{C / S}\right)_{\left(\alpha_{1}, \ldots, \alpha_{i}+k r_{i}, \ldots, \alpha_{n}\right)}$ dans $\left(\operatorname{Pic}_{C / S}\right)_{\underline{\alpha}}$. La loi de groupe est évidente.

Remarque 5.4.7 Si l'on ne tient pas compte de la structure de groupe, on voit que $\operatorname{Pic}_{\mathscr{C} / S}$ s'identifie à une union disjointe de $r_{1} \ldots r_{n}$ copies de $\mathrm{Pic}_{C / S}$. En particulier la composante neutre de $\operatorname{Pic}_{\mathscr{C} / S}$ est la même que celle de $\operatorname{Pic}_{C / S}$. Autrement dit, le morphisme $\operatorname{de~} \operatorname{Pic}_{C / S}$ vers $\operatorname{Pic}_{\mathscr{C} / S}$ induit un isomorphisme naturel : $\operatorname{Pic}_{C / S}^{0} \stackrel{\sim}{\longrightarrow} \operatorname{Pic}_{\mathscr{C} / S}^{0}$.

Remarque 5.4.8 Pour décrire le champ de Picard de $\mathscr{C} / S$, on commence par remarquer que l'on a une suite exacte ${ }^{9}$ de champs de Picard:

$$
1 \longrightarrow \mathscr{P} i c(C / S) \stackrel{\pi^{*}}{\longrightarrow} \mathscr{P} i c(\mathscr{C} / S) \longrightarrow \prod_{i=1}^{n} \mathbb{Z} / r_{i} \mathbb{Z} \longrightarrow 0 \text {. }
$$

De la même manière que pour le foncteur de Picard, le champ $\mathscr{P}$ ic $(\mathscr{C} / S)$ s'identifie au quotient de $\mathscr{P} i c(C / S) \times{ }_{S} \mathbb{Z}^{n}$ par les relations $t_{i}^{r_{i}}=l_{i}$ (où les $t_{i}$ désignent les générateurs canoniques de $\left.\mathbb{Z}^{n}\right)$. En particulier, si l'on oublie la structure de groupe, $\mathscr{P} i c(\mathscr{C} / S)$ s'identifie à une union disjointe de $r_{1} \ldots r_{n}$ copies de $\mathscr{P}_{i c}(C / S)$.

\section{Annexes}

Ainsi qu'il a été dit en introduction, la présente annexe rassemble les résultats relatifs à la cohomologie des faisceaux sur les champs algébriques nécessaires au texte principal. Pour éviter que cet article ne devienne démesurément long, les résultats sont donnés ici sans démonstration. On pourra trouver ces dernières, ainsi que des commentaires plus fournis, dans la thèse [15] de l'auteur. Voici en résumé la liste des sujets qui y sont abordés.

Dans la première section, nous rappelons les définitions de base et nous vérifions que sur un champ de Deligne-Mumford, les groupes de cohomologie lisse-étale coïncident avec les groupes de cohomologie étale. Puis nous introduisons un nouveau site, le site lisse-lisse champêtre, dont les objets sont les morphismes représentables et lisses $\mathscr{U} \rightarrow \mathscr{X}$ de champs algébriques. Il définit le même topos que le site lisse-étale mais présente au moins deux avantages : il se comporte mieux vis-à-vis des images directes et il a un objet final.

C'est au départ la nécessité de disposer de techniques de descente cohomologique à la Deligne-Saint-Donat qui a motivé le travail du premier paragraphe de la section A.2. Nous avions en particulier besoin d'un analogue pour les champs algébriques de la suite spectrale de descente relative à un morphisme lisse et surjectif de schémas. Il s'est finalement avéré que l'introduction du site lisse-lisse champêtre rendait ce résultat presque trivial (voir proposition A.2.1). Nous décrivons ensuite une classe de faisceaux acycliques adaptée aux particularités du site lisse-étale. Ces faisceaux «Llcacycliques » nous sont surtout utiles pour obtenir la suite spectrale de Leray relative

$\overline{9}$ en un sens analogue à celui donné dans le dernier paragraphe de 5.3. 
à un morphisme de champs algébriques (thm. A.2.8). Nous montrons aussi que les images directes supérieures d'un faisceau lisse-étale abélien peuvent être calculées comme l'on imagine ( $c f$. prop. A.2.5).

Le premier paragraphe de la section A.3 est consacré au changement de base plat. Le second aux extensions infinitésimales. Il est d'usage, lorsque $i: X \rightarrow \widetilde{X}$ est une extension infinitésimale, d'identifier les catégories de faisceaux Zariski sur $X$ et sur $\widetilde{X}$. Ceci est tout à fait légitime puisque $X$ et $\widetilde{X}$ ont le même espace topologique sous-jacent. Mieux : le foncteur qui à un ouvert étale $\widetilde{U}$ de $\widetilde{X}$ associe l'ouvert étale $\widetilde{U} \times \widetilde{X} X$ de $X$ définit une équivalence entre les sites étales de $\widetilde{X}$ et de $X$, ce qui permet d'identifier aussi les faisceaux étales. Il faut faire nettement plus attention avec la topologie lisseétale. On peut en effet vérifier facilement que le foncteur ci-dessus n'est même pas fidèle. Heureusement, on peut tout de même identifier les groupes de cohomologie des faisceaux abéliens sur $X$ et sur $\widetilde{X}$ via le foncteur $i_{*}$ (cf. A.3.5). Cette section contient également un résultat analogue pour les images directes supérieures. Nous donnons un résultat de descente pour les champs algébriques, puis nous décrivons les torseurs du topos lisse-étale et montrons que, dans le cas particulier d'un groupe lisse sur la base $S$, le $H^{1}$ au sens des foncteurs dérivés coïncide avec le groupe des classes de torseurs.

La section B est dévolue à la cohomologie plate sur les champs algébriques. Nous donnons principalement deux résultats. D'une part la suite spectrale qui relie la cohomologie plate à la cohomologie lisse-étale, et d'autre part la généralisation aux champs algébriques du théorème de Grothendieck suivant lequel dans le cas d'un groupe lisse, la cohomologie plate coïncide avec la cohomologie étale (cf. [2], exposé VI, paragraphe 11).

\section{A Cohomologie lisse-étale sur les champs algébriques}

\section{A.1 Topos lisse-étale et cohomologie des faisceaux}

Rappelons brièvement, pour la commodité du lecteur, la définition du site lisse-étale d'un champ algébrique donnée au chapitre 12 de [34].

Définition A.1.1 $[34,12.1]$ Soit $\mathscr{X}$ un $S$-champ algébrique. On appelle site lisseétale de $\mathscr{X}$ et on note Lis-ét( $\mathscr{X})$ le site défini comme suit.

Les ouverts lisses-étales de $\mathscr{X}$ sont les couples $(U, u)$ où $U$ est un $S$-espace algébrique et $u: U \rightarrow \mathscr{X}$ un morphisme représentable et lisse. Une flèche entre deux tels ouverts $(U, u)$ et $(V, v)$ est un couple $(\varphi, \alpha)$ faisant 2-commuter le diagramme suivant :

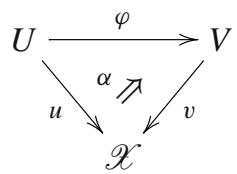

Une famille couvrante de $(U, u)$ est une collection de morphismes

$$
\left(\left(\varphi_{i}, \alpha_{i}\right):\left(U_{i}, u_{i}\right) \longrightarrow(U, u)\right)_{i \in I}
$$


telle que le 1-morphisme d'espaces algébriques

$$
\coprod_{i \in I} \varphi_{i}: \coprod_{i \in I} U_{i} \longrightarrow U
$$

soit étale et surjectif.

Le site étale de $\mathscr{X}$, noté Ét( $\mathscr{X})$, est la sous-catégorie pleine de Lis-ét( $\mathscr{X})$ dont les objets sont les couples $(U, u)$ où $u$ est un morphisme étale, munie de la topologie induite par celle de Lis-ét $(\mathscr{X})$.

On notera $\mathscr{O}_{\mathscr{X}}$ le faisceau structural de $\mathscr{X}$ défini de manière évidente. Si $\mathscr{A}$ est un faisceau d'anneaux sur Lis-ét $(\mathscr{X})$, on notera $\operatorname{Mod}_{\mathscr{A}}(\mathscr{X})$ la catégorie des faisceaux de $\mathscr{A}$-modules sur le site lisse-étale de $\mathscr{X}$, ou plus simplement $\operatorname{Mod}(\mathscr{X})$ lorsque $\mathscr{A}=\mathscr{O}_{\mathscr{X}}$. La catégorie des faisceaux abéliens sera notée $\mathrm{Ab}(\mathscr{X})$. Les références usuelles ([3] II (6.7) et [24] théorème (1.10.1)) permettent de vérifier que la catégorie $\operatorname{Mod}_{\mathscr{A}}(\mathscr{X})$ est une catégorie abélienne avec suffisamment d'objets injectifs. En particulier il en est ainsi des catégories $\operatorname{Mod}(\mathscr{X})$ et $\operatorname{Ab}(\mathscr{X})$.

On rappelle que le foncteur «sections globales » est défini de la manière suivante. Si $\mathscr{F}$ est un faisceau lisse-étale sur $\mathscr{X}$, l'ensemble $\Gamma(\mathscr{X}, \mathscr{F})$ est l'ensemble des familles $\left(s_{(U, u)}\right)$ de sections de $\mathscr{F}$ sur les $(U, u) \in$ ob Lis-ét $(\mathscr{X})$ qui sont compatibles aux flèches de restriction en un sens évident (cf. [34] (12.5.3)). On vérifie immédiatement que le foncteur $\Gamma(\mathscr{X},):. \mathrm{Ab}(\mathscr{X}) \rightarrow \mathrm{Ab}$ est exact à gauche. On définit alors $H^{i}(\mathscr{X},$.$) comme étant le i$ ème foncteur dérivé à droite de $\Gamma(\mathscr{X},$.$) .$

Il résulte maintenant de [4] V (3.5) que sur la catégorie $\operatorname{Mod}(\mathscr{X})$, le foncteur $H^{i}(\mathscr{X},$.$) coïncide avec le i$ ème foncteur dérivé à droite de $\Gamma(\mathscr{X},):. \operatorname{Mod}(\mathscr{X}) \rightarrow \mathrm{Ab}$.

Lemme A.1.2 [15, A.1.1.5] Soit $\mathscr{X}$ un S-champ de Deligne-Mumford et soient $\mathscr{F}$, $\mathscr{G}$ des faisceaux lisses-étales d'ensembles (resp. de groupes abéliens) sur $\mathscr{X}$. On suppose que $\mathscr{F}$ est cartésien (voir la définition dans [34] (12.3)). Alors l'application

$$
\operatorname{Hom}(\mathscr{F}, \mathscr{G}) \longrightarrow \operatorname{Hom}\left(\mathscr{F}_{\text {ét }}, \mathscr{G}_{\text {ét }}\right)
$$

induite par le foncteur d'inclusion Ét $(\mathscr{X}) \hookrightarrow$ Lis-ét( $\mathscr{X})$ est bijective.

Remarque A.1.3 Le résultat est faux si l'on ne suppose pas $\mathscr{F}$ cartésien. Il suffit de considérer un faisceau lisse-étale abélien $\mathscr{F}$ non nul tel que $\mathscr{F}_{\text {ét }}$ soit nul. Alors, dans $\operatorname{Hom}(\mathscr{F}, \mathscr{F})$ on a au moins deux éléments distincts, à savoir l'identité et le morphisme nul, tandis que $\operatorname{Hom}\left(\mathscr{F}_{\text {ét }}, \mathscr{F}_{\text {ét }}\right)$ est réduit à zéro. Pour exhiber un tel faisceau, on peut par exemple prendre $\mathscr{X}=$ Spec $k$, le spectre d'un corps, et poser $\mathscr{F}(U, u)=\Omega_{U / k}$.

Corollaire A.1.4 [15, A.1.1.7] Soient $\mathscr{X}$ un S-champ de Deligne-Mumford et $\mathscr{F}$ un faisceau lisse-étale sur $\mathscr{X}$. Alors le morphisme canonique

$$
\Gamma_{\text {lis-ét }}(\mathscr{X}, \mathscr{F}) \longrightarrow \Gamma_{\text {ét }}(\mathscr{X}, \mathscr{F})
$$

est un isomorphisme. 
Corollaire A.1.5 [15, A.1.1.8] Soit $\mathscr{X}$ un S-champ de Deligne-Mumford, et soit $\mathscr{F}$ un objet injectif de la catégorie des faisceaux lisses-étales abéliens sur $\mathscr{X}$. Alors la restriction $\mathscr{F}_{\text {ét }}$ de $\mathscr{F}$ au site étale de $\mathscr{X}$ est un objet injectif de la catégorie des faisceaux étales abéliens.

Proposition A.1.6 [15, A.1.1.9] Soient $\mathscr{X}$ un S-champ de Deligne-Mumford et $\mathscr{F} \in$ $\operatorname{Ab}(\mathscr{X})$ un faisceau lisse-étale abélien sur $\mathscr{X}$. On note $\mathscr{F}$ ét la restriction de $\mathscr{F}$ au site étale de $\mathscr{X}$. Alors pour tout $q \geq 0$ on a un isomorphisme canonique:

$$
H_{\text {lis-ét }}^{q}(\mathscr{X}, \mathscr{F}) \stackrel{\sim}{\longrightarrow} H_{\text {ét }}^{q}\left(\mathscr{X}, \mathscr{F}_{\text {ét }}\right)
$$

FONCTORIALITÉ DU TOPOS LISSE- ÉTALE

Si $f: \mathscr{X} \rightarrow \mathscr{Y}$ est un 1-morphisme de $S$-champs algébriques, on lui associe un couple de foncteurs adjoints $\left(f^{-1}, f_{*}\right)$ comme dans [34], (12.5). Comme on pourra le lire bientôt dans la prochaine édition de [34], ou dès aujourd'hui dans [40], il serait erroné de penser que le couple $\left(f^{-1}, f_{*}\right)$ est toujours un morphisme de topos. Il peut en effet arriver que le foncteur $f^{-1}$ ne soit pas exact, même lorsque $\mathscr{X}$ et $\mathscr{Y}$ sont des schémas. Pour s'en convaincre on consultera les références citées. C'est toutefois le cas dès que $f$ est lisse.

Le foncteur $f_{*}: \mathrm{Ab}(\mathscr{X}) \rightarrow \mathrm{Ab}(\mathscr{Y})$ est exact à gauche puisqu'il a un adjoint à gauche. On peut donc définir les foncteurs dérivés $R^{q} f_{*}: \operatorname{Ab}(\mathscr{X}) \rightarrow \operatorname{Ab}(\mathscr{Y})$.

\section{LE SITE LISSE- LISSE CHAMPÊTRE D'UN CHAMP ALGÉBRIQUE}

Pour un certain nombre de considérations techniques, le site lisse-étale défini dans [34] ne contient pas suffisamment d'ouverts pour être vraiment commode. En effet, lorsque $f$ n'est pas représentable, le foncteur $f_{*}$ n'est pas induit par une application continue Lis-ét $(\mathscr{X}) \rightarrow$ Lis-ét( $\mathscr{Y})$, ce qui pose problème par exemple lorsque l'on essaye de calculer les foncteurs images directes supérieures $R^{q} f_{*}$ [voir le paragraphe A.2]. C'est la raison pour laquelle nous introduisons un site un peu plus gros, qui ne présentera plus les mêmes inconvénients. Nous démontrons ensuite (A.1.9) que le topos qu'il définit est équivalent au topos lisse-étale. Le choix de la topologie lisse plutôt qu'étale pour ce site est essentiellement dû au fait que pour la topologie étale, les « ouverts lisses champêtres » ne sont pas toujours recouverts par un espace algébrique.

Soit $\mathscr{X}$ un $S$-champ algébrique. On définit la 2-catégorie des ouverts lisses champêtres de la manière suivante. Les objets sont les couples $(\mathscr{U}, u)$ où $\mathscr{U}$ est un $S$-champ algébrique et $u: \mathscr{U} \rightarrow \mathscr{X}$ est un morphisme représentable et lisse. Un 1-morphisme entre deux tels ouverts $(\mathscr{U}, u)$ et $(\mathscr{V}, v)$ est un couple $(\varphi, \alpha)$ où $\varphi: \mathscr{U} \rightarrow \mathscr{V}$ est un 1-morphisme (automatiquement représentable!) de $S$-champs algébriques et $\alpha: u \Rightarrow v \circ \varphi$ est un 2-isomorphisme.

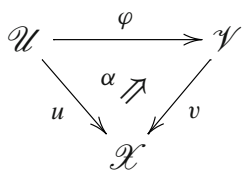


Si $(\varphi, \alpha)$ et $(\psi, \beta)$ sont deux 1-morphismes de $(\mathscr{U}, u)$ dans $(\mathscr{V}, v)$, un 2-morphisme entre $(\varphi, \alpha)$ et $(\psi, \beta)$ est un 2-isomorphisme $\gamma: \varphi \Rightarrow \psi$ tel que $\beta=\left(v_{*} \gamma\right) \circ \alpha$.

Lemme A.1.7 [15, A.1.3.1] Soient $(\mathscr{U}, u)$ et $(\mathscr{V}, v)$ deux ouverts lisses champêtres. Alors la catégorie des morphismes de $(\mathscr{U}, u)$ dans $(\mathscr{V}, v)$ est équivalente à une catégorie discrète.

À l'avenir on identifiera $\operatorname{Hom}((\mathscr{U}, u),(\mathscr{V}, v))$ à une catégorie discrète équivalente et on parlera de l'ensemble des morphismes de $(\mathscr{U}, u)$ dans $(\mathscr{V}, v)$, et de la catégorie des ouverts lisses champêtres. Il est clair que cette catégorie admet des produits fibrés.

Définition A.1.8 [15, A.1.3.2] On appelle site lisse-lisse champêtre, et on note $\operatorname{Llc}(\mathscr{X})$, la catégorie des ouverts lisses champêtres de $\mathscr{X}$ munie de la topologie engendrée par la prétopologie pour laquelle les familles couvrantes sont les familles de morphismes

$$
\left(\left(\mathscr{U}_{i}, u_{i}\right) \longrightarrow(\mathscr{U}, u)\right)_{i \in I}
$$

telles que le morphisme (automatiquement représentable)

$$
\coprod_{i \in I} u_{i}: \coprod_{i \in I} \mathscr{U}_{i} \longrightarrow \mathscr{U}
$$

soit lisse et surjectif.

Proposition A.1.9 [15, A.1.3.3]

1) La topologie de Lis-ét $(\mathscr{X})$ est égale à la topologie engendrée par la prétopologie dite lisse, pour laquelle les familles couvrantes sont les familles de morphismes $\left(\left(U_{i}, u_{i}\right) \rightarrow(U, u)\right)_{i \in I}$ telles que le morphisme $\coprod_{i \in I} u_{i}: \coprod_{i \in I} U_{i} \rightarrow U$ soit lisse et surjectif.

2) Le foncteur d'inclusion $\operatorname{Lis-ét~}(\mathscr{X}) \hookrightarrow \operatorname{Llc}(\mathscr{X})$ induit une équivalence de topos de la catégorie des faisceaux sur le site lisse-lisse champêtre vers la catégorie des faisceaux sur Lis-ét $(\mathscr{X})$.

Remarque A.1.10 Notons $\mathscr{X}_{\text {Llc }}$ le topos des faisceaux $\operatorname{sur} \operatorname{Llc}(\mathscr{X})$. Via l'équivalence de topos ci-dessus, si $f: \mathscr{X} \longrightarrow \mathscr{Y}$ est un 1-morphisme de $S$-champs algébriques, le foncteur $f_{*}$ est simplement donné par $\left(f_{*} \mathscr{F}\right)(\mathscr{U}, u)=\mathscr{F}(\mathscr{X} \times \mathscr{Y} \mathscr{U}, \operatorname{pr} \mathscr{X})$ pour tout ouvert lisse champêtre $u: \mathscr{U} \longrightarrow \mathscr{Y}$ de $\mathscr{Y}$. Le foncteur «sections globales » est quant à lui donné par $\Gamma(\mathscr{X}, \mathscr{F})=\mathscr{F}(\mathscr{X}, \operatorname{Id} \mathscr{X})$.

Remarque A.1.11 Si $u: \mathscr{U} \rightarrow \mathscr{X}$ est un morphisme représentable et lisse de $S$-champs algébriques, et si $\mathscr{A}$ est un anneau du topos $\mathscr{X}_{\mathrm{Llc}}$, le foncteur

$$
u^{*}:\left\{\begin{aligned}
\operatorname{Mod}_{\mathscr{A}}(\mathscr{X}) & \longrightarrow \operatorname{Mod}_{\mathscr{A}_{(\mathscr{U}, u)}}(\mathscr{U}) \\
\mathscr{F} & \longmapsto \mathscr{F}_{(\mathscr{U}, u)}
\end{aligned}\right.
$$


où $\mathscr{F}_{(\mathscr{U}, u)}$ (resp. $\left.\mathscr{A}_{(\mathscr{U}, u)}\right)$ désigne la restriction de $\mathscr{F}($ resp. $\mathscr{A}$ ) au site lisse-lisse champêtre de $\mathscr{U}$, commute aux limites projectives et inductives arbitraires. Il a donc un adjoint à gauche $u_{\text {! }}$, et de plus cet adjoint est exact (tout ceci résulte de [3, IV (11.3.1)]). Par suite $u^{*}$ transforme les objets injectifs en objets injectifs (voir aussi [4, V (2.2])).

\section{A.2 Suites spectrales}

\section{LA SUITE SPECTRALE RELATIVE A UN RECOUVREMENT}

Soit $P: \mathscr{U}^{0} \rightarrow \mathscr{X}$ un morphisme représentable, lisse et surjectif de $S$-champs algébriques. On note

$$
\begin{aligned}
\mathscr{U}^{1} & =\mathscr{U}^{0} \times \mathscr{X} \mathscr{U}^{0} \\
\mathscr{U}^{2} & =\mathscr{U}^{0} \times \mathscr{X} \mathscr{U}^{0} \times \mathscr{X}^{0} \mathscr{U}^{0} \\
& \vdots \\
\mathscr{U}^{n} & =\mathscr{U}^{0} \times \mathscr{X} \cdots \times \mathscr{X}^{0} \quad(n+1 \text { fois })
\end{aligned}
$$

Les $\mathscr{U}^{n}$ forment avec les diagonales partielles et les projections un $S$-champ algébrique simplicial muni d'une augmentation vers $\mathscr{X}$ :

$$
\ldots \quad \mathscr{U}^{n} \quad \ldots \quad \mathscr{U}^{2} \underset{\longleftrightarrow}{\longleftrightarrow} \mathscr{U}^{1} \underset{\longleftrightarrow}{\longleftrightarrow} \mathscr{U}^{0} \longrightarrow \mathscr{X}
$$

Soit $\mathscr{F}$ un faisceau abélien sur le site lisse-étale de $\mathscr{X}$. On note $\mathscr{F}^{i}$ la restriction de $\mathscr{F}$ au site lisse-étale de $\mathscr{U}^{i}$. On cherche alors à calculer la cohomologie de $\mathscr{F}$ en fonction de la cohomologie des $\mathscr{F}^{i}$ sur les $\mathscr{U}^{i}$. On peut associer au champ algébrique simplicial ci-dessus un complexe de Čech de la manière suivante. Pour $n \geq 2$, on note $p_{1 \ldots} \hat{l}_{\text {...n }}$, où la notation $\hat{l}$ signifie que l'indice $l$ est omis, la projection $\mathscr{U}^{n-1} \rightarrow \mathscr{U}^{n-2}$ qui «oublie » le facteur d'indice $l$ de $\mathscr{U}^{n-1}$. Par exemple $p_{1}$ et $p_{2}$ désignent respectivement les première et seconde projections de $\mathscr{U}^{0} \times \mathscr{X}^{0} \mathscr{U}^{0}$ sur $\mathscr{U}^{0}$. On définit alors le complexe de Čech $S\left(H^{q}\right)$ comme étant le complexe:

$$
H^{q}\left(\mathscr{U}^{0}, \mathscr{F}^{0}\right) \stackrel{d^{0}}{\longrightarrow} H^{q}\left(\mathscr{U}^{1}, \mathscr{F}^{1}\right) \stackrel{d^{1}}{\longrightarrow} \cdots \longrightarrow H^{q}\left(\mathscr{U}^{p}, \mathscr{F}^{p}\right) \stackrel{d^{p}}{\longrightarrow} \cdots
$$

avec

$$
\begin{aligned}
d^{0} & =p_{2}^{*}-p_{1}^{*} \\
d^{1} & =p_{23}^{*}-p_{13}^{*}+p_{12}^{*} \\
& \vdots \\
d^{p} & =\sum_{l=1}^{p+2}(-1)^{l+1} p_{1 \cdots \hat{l} \cdots(p+2)}^{*}
\end{aligned}
$$


On désigne par $\check{H}^{p}\left(H^{q}\left(\mathscr{U}^{\bullet}, \mathscr{F} \bullet\right)\right)$ l'homologie en degré $p$ de ce complexe:

$$
\check{H}^{p}\left(H^{q}\left(\mathscr{U}^{\bullet}, \mathscr{F}^{\bullet}\right)\right)=\frac{\operatorname{Ker} d^{p}}{\operatorname{Im} d^{p-1}} .
$$

Le résultat suivant, qui donne la suite spectrale reliant la « cohomologie de Čech » relativement à la famille couvrante $P: \mathscr{U}^{0} \rightarrow \mathscr{X}$, à la cohomologie lisse-étale de $\mathscr{F}$ sur $\mathscr{X}$, est essentiellement trivial ( $c f$. [15]). Il ne fait pas appel aux techniques de descente cohomologique de Deligne présentées par Saint-Donat dans l'exposé V bis de [4] : grâce à l'introduction du site lisse-lisse champêtre, il s'agit juste de la « suite spectrale de Cartan-Leray relative à un recouvrement » [4, V (3.3)] .

Proposition A.2.1 Reprenons les hypothèses et notations précédentes. Il existe une suite spectrale (fonctorielle):

$$
E_{2}^{p, q}=\check{H}^{p}\left(H^{q}\left(\mathscr{U}^{\bullet}, \mathscr{F}^{\bullet}\right)\right) \Rightarrow H^{p+q}(\mathscr{X}, \mathscr{F}) .
$$

\section{FAISCEAUX ACYCLIQUES}

Soit $f: \mathscr{X} \rightarrow \mathscr{Y}$ un morphisme de $S$-champs algébriques. Dans la mesure où le couple de foncteurs adjoints $\left(f^{-1}, f_{*}\right)$ n'est pas un morphisme de topos, il n'est pas évident a priori que le foncteur $f_{*}$ transforme les faisceaux injectifs en faisceaux injectifs. Il nous faudra pourtant montrer, pour obtenir la suite spectrale de Leray relative à un morphisme de champs algébriques (cf. paragraphe A.2), que le foncteur $f_{*}$ transforme les faisceaux injectifs en faisceaux acycliques pour le foncteur « sections globales ». L'usage de faisceaux «flasques » en un certain sens va nous permettre de résoudre ce problème. La principale difficulté réside dans le choix de la classe de faisceaux que l'on considère ( $c f$. [15]).

Définition A.2.2 [4, V 4.2] Soit $\mathscr{X}$ un $S$-champ algébrique et soit $\mathscr{F}$ un faisceau lisseétale abélien. On dit que $\mathscr{F}$ est Llc-acyclique si pour tout morphisme représentable et lisse $u: \mathscr{U} \rightarrow \mathscr{X}$ et pour tout $q>0$, le groupe $H^{q}\left(\mathscr{U}, \mathscr{F}_{(\mathscr{U}, u)}\right)$ est nul.

Remarque A.2.3 S'il est évident que les faisceaux flasques au sens de SGA 4 sont Llc-acycliques, il n'y a aucune raison a priori pour que la réciproque soit vraie. Pour quelques commentaires sur ce genre de questions, on pourra consulter [4] V 4.6 et 4.13. Par ailleurs il est évident que les faisceaux injectifs sont flasques, donc aussi Llc-acycliques.

La proposition suivante, quoique fortement inspirée de la proposition V 4.3 de [4], en diffère légèrement.

Proposition A.2.4 [15, A.1.5.3] Soient $\mathscr{X}$ un S-champ algébrique, et $\mathscr{F}$ un faisceau sur $\operatorname{Llc}(\mathscr{X})$. Les propositions suivantes sont équivalentes:

(1) $\mathscr{F}$ est Llc-acyclique;

(2) pour toute famille couvrante $\left(\left(\mathscr{U}^{\prime}, u^{\prime}\right) \rightarrow(\mathscr{U}, u)\right)$ dans $\operatorname{Llc}(\mathscr{X})$, et pour tout $q>0$, le groupe $H^{q}\left(\mathscr{U}^{\prime} / \mathscr{U}, \mathscr{F}\right)$ est nul (où $H^{q}\left(\mathscr{U}^{\prime} / \mathscr{U}, \mathscr{F}\right)$ désigne le qème groupe de cohomologie de Čech de $\mathscr{F}$, aussi noté $\check{H}^{q}\left(H^{0}\left(\mathscr{U}^{\prime \bullet}, \mathscr{F}_{(\mathscr{U}, u)}^{\bullet}\right)\right)$ dans la section précédente). 
IMAGES DIRECTES SUPÉRIEURES ET SUITE SPECTRALE DE LERAY RELATIVE A UN MORPHISME DE CHAMPS ALGÉBRIQUES

La proposition suivante montre que les images directes supérieures se calculent comme l'on pense. Leurs restrictions au site étale de $\mathscr{Y}$ aussi (voir l'énoncé, plus précis, donné dans [15]).

\section{Proposition A.2.5 ([15, A.1.6.1])}

1) Soit $f: \mathscr{X} \rightarrow \mathscr{Y}$ un morphisme de S-champs algébriques et soit $\mathscr{F}$ un faisceau abélien sur Lis-ét $(\mathscr{X})$. Alors le faisceau $R^{q} f_{*} \mathscr{F}$ est le faisceau associé au préfaisceau qui à tout ouvert lisse-étale $(U, u)$ de $\mathscr{Y}$ associe $H^{q}(\mathscr{X} \times \mathscr{Y}$ $\left.U, \mathscr{F}_{\left(\mathscr{X} \times \mathscr{Y} U, \mathrm{pr}_{\mathscr{X}}\right)}\right)$.

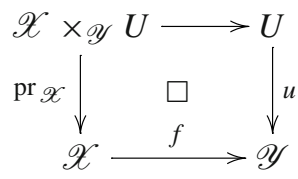

2) $\operatorname{Si} \mathscr{Y}=\operatorname{Spec} A$ est un schéma affine et si $\mathscr{X}$ est quasi-compact, alors pour tout faisceau quasi-cohérent $\mathscr{F}$ sur $\mathscr{X}$ :

$$
H^{0}\left(\operatorname{Spec} A, R^{q} f_{*} \mathscr{F}\right) \simeq H^{q}(\mathscr{X}, \mathscr{F})
$$

Proposition A.2.6 ([15, A.1.6.2]) La restriction du foncteur $R^{q} f_{*}$ à la catégorie $\operatorname{Mod}(\mathscr{X})$ coïncide avec le $q^{i e ̀ m e}$ foncteur dérivé à droite de $f_{*}: \operatorname{Mod}(\mathscr{X}) \rightarrow \operatorname{Mod}(\mathscr{Y})$.

Lemme A.2.7 ([15, A.1.6.3]) Le foncteur $f_{*}: \mathrm{Ab}(\mathscr{X}) \rightarrow \mathrm{Ab}(\mathscr{Y})$ préserveles faisceaux Llc-acycliques. En particulier il transforme les faisceaux injectifs en faisceaux acycliques pour le foncteur $\Gamma(\mathscr{Y},$.$) .$

Théorème A.2.8 (suite spectrale de Leray [15, A.1.6.4]) Soit $f: \mathscr{X} \rightarrow \mathscr{Y}$ un morphisme de $S$-champs algébriques, et soit $\mathscr{F}$ un faisceau lisse-étale abélien sur $\mathscr{X}$. Il existe une suite spectrale:

$$
H^{p}\left(\mathscr{Y}, R^{q} f_{*} \mathscr{F}\right) \Rightarrow H^{p+q}(\mathscr{X}, \mathscr{F}) .
$$

Proposition A.2.9 ([15, A.1.6.5]) Soient $f: \mathscr{X} \rightarrow \mathscr{Y}$ et $g: \mathscr{Y} \rightarrow \mathscr{Z}$ des morphismes de $S$-champs algébriques et soit $\mathscr{F}$ un faisceau lisse-étale abélien sur $\mathscr{X}$. Alors on a une suite spectrale:

$$
R^{p} g_{*} R^{q} f_{*} \mathscr{F} \Rightarrow R^{p+q}(g \circ f)_{*} \mathscr{F} .
$$

\section{A.3 Comparaison de cohomologies}

\section{COHOMOLOGIE ET CHANGEMENT DE BASE}

La démonstration de la proposition A.3.3 ci-dessous (donc celle de son corollaire A.3.4) utilise des hyperrecouvrements. La définition que, pour la commodité du 
lecteur, nous rappelons ci-dessous, est plus simple en apparence que celle présentée dans [4]. Elle lui est équivalente dès que la catégorie sous-jacente à $C$ admet des sommes directes arbitraires (en effet les objets semi-représentables sont alors représentables), ce que nous supposons dès à présent.

Définition A.3.1 (cf. [4] V (7.3.1.2)) Soient $C$ un site (admettant des sommes directes arbitraires), $X$ un objet de $C$ et $U^{\bullet} \rightarrow X$ un objet simplicial de $C$ muni d'une augmentation vers $X$, ou de manière équivalente un objet simplicial de $C / X$. On dit que $U^{\bullet}$ est un hyperrecouvrement de $X$ s'il possède les propriétés suivantes:

(1) Pour tout entier $n \geq 0$ le morphisme canonique

$$
U^{n+1} \longrightarrow\left(\operatorname{cosq}_{n}\left(\operatorname{sq}_{n} U^{\bullet}\right)\right)_{n+1}
$$

est un morphisme couvrant.

(2) Le morphisme $U^{0} \rightarrow X$ est couvrant.

Remarque A.3.2 Dans la définition précédente, $\mathrm{sq}_{n}$ désigne le foncteur qui à un objet simplicial associe son tronqué à l'ordre $n$. Le foncteur $\operatorname{cosq}_{n}$ est l'adjoint à droite de $\mathrm{sq}_{n}$. Enfin $\left(\operatorname{cosq}_{n}\left(\mathrm{sq}_{n} U^{\bullet}\right)\right)_{n+1}$ désigne le terme d'indice $n+1$ de l'objet simplicial $\operatorname{cosq}_{n}\left(\mathrm{sq}_{n} U^{\bullet}\right)$.

Si $U^{\bullet} \rightarrow X$ est un hyperrecouvrement de $X$ et si $\mathscr{F}$ est un faisceau abélien sur $X$, on lui associe un complexe

$$
0 \longrightarrow H^{q}\left(U^{0}, \mathscr{F}^{0}\right) \longrightarrow H^{q}\left(U^{1}, \mathscr{F}^{1}\right) \longrightarrow \cdots
$$

de manière tout à fait analogue à ce qui a été fait dans la section A.2 (où $\mathscr{F}^{i}$ désigne la restriction de $\mathscr{F}$ à $\left.U^{i}\right)$. On note encore $\check{H}^{p}\left(H^{q}\left(U^{\bullet}, \mathscr{F} \bullet\right)\right)$ l'homologie en degré $p$ de ce complexe. On a alors une suite spectrale ([4] V (7.4.0.3)) :

$$
\check{H}^{p}\left(H^{q}\left(U^{\bullet}, \mathscr{F}^{\bullet}\right)\right) \Rightarrow H^{p+q}(X, \mathscr{F}) .
$$

Proposition A.3.3 [15, A.1.7.3] Soit $\mathscr{X}$ un $S$-champ algébrique quasi-compact, avec $S=\operatorname{Spec} A$ affine, et soit $\mathscr{F}$ un faisceau quasi-cohérent sur $\mathscr{X}$. Soient $A^{\prime}$ une A-algèbre plate, $S^{\prime}=\operatorname{Spec} A^{\prime}$, et $\mathscr{X}^{\prime}=\mathscr{X} \times{ }_{S} S^{\prime}$. On note $\mathscr{F}^{\prime}$ l'image inverse de $\mathscr{F}$ sur $\mathscr{X}^{\prime}$. Alors pour tout $q \geq 0$ le morphisme naturel

$$
H^{q}(\mathscr{X}, \mathscr{F}) \otimes_{A} A^{\prime} \longrightarrow H^{q}\left(\mathscr{X}^{\prime}, \mathscr{F}^{\prime}\right)
$$

est un isomorphisme.

Proposition A.3.4 [15, A.1.7.4] Soit $f: \mathscr{X} \rightarrow \mathscr{Y}$ un morphisme quasi-compact de S-champs algébriques, et soit $\mathscr{F}$ un faisceau quasi-cohérent sur $\mathscr{X}$. Soit u : 
$\mathscr{Y}^{\prime} \rightarrow \mathscr{Y}$ un morphisme plat de changement de base.

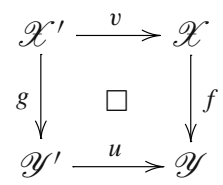

Alors pour tout $q \geq 0$ le morphisme naturel

$$
u^{*} R^{q} f_{*} \mathscr{F} \longrightarrow\left(R^{q} g_{*}\right)\left(v^{*} \mathscr{F}\right)
$$

est un isomorphisme.

\section{COHOMOLOGIE ET EXTENSIONS INFINITÉSIMALES}

Lemme A.3.5 [15, A.1.8.1] Soit $i: \mathscr{X} \rightarrow \widetilde{\mathscr{X}}$ une immersion fermée de champs algébriques définie par un idéal quasi-cohérent de $\widetilde{\mathscr{X}}$ de carré nul, et soit $\mathscr{F}$ un faisceau abélien sur $\mathscr{X}$. Alors pour tout $q>0$ le faisceau $R^{q} i_{*} \mathscr{F}$ est nul. En particulier pour tout $n$ le morphisme naturel:

$$
H^{n}\left(\widetilde{\mathscr{X}}, i_{*} \mathscr{F}\right) \longrightarrow H^{n}(\mathscr{X}, \mathscr{F})
$$

est un isomorphisme.

Corollaire A.3.6 [15, A.1.8.3] Soit

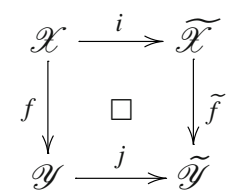

un carré 2-cartésien de $S$-champs algébriques, dans lequel on suppose que $i$ et $j$ sont des immersions fermées définies par des idéaux quasi-cohérents de carrés nuls. Soit $\mathscr{F}$ un faisceau abélien sur $\mathscr{X}$. Alors on a pour tout $p$ un isomorphisme canonique:

$$
R^{p} \widetilde{f}_{*}\left(i_{*} \mathscr{F}\right) \stackrel{\sim}{\longrightarrow} j_{*} R^{p} f_{*} \mathscr{F} .
$$

\section{UN RÉSULTAT DE DESCENTE}

Le théorème suivant n'est autre qu'un résultat de descente pour les champs algébriques. Il est utilisé au paragraphe suivant.

Théorème A.3.7 [15, A.1.9.1] Soit $\mathscr{F}$ un faisceau $\operatorname{sur} \operatorname{Llc}(\mathscr{X})$ qui, localement pour la topologie lisse sur $\mathscr{X}$, est représentable. Alors $\mathscr{F}$ est représentable par un unique objet $(\mathscr{P}, p)$ de $\operatorname{Llc}(\mathscr{X})$. Autrement dit il existe un morphisme représentable et lisse de $S$-champs algébriques $p: \mathscr{P} \rightarrow \mathscr{X}$ qui représente $\mathscr{F}$. 
Remarque A.3.8 L'hypothèse sur $\mathscr{F}$ signifie qu'il existe une présentation $x_{0}: X_{0} \rightarrow \mathscr{X}$ de $\mathscr{X}$ telle que la restriction de $\mathscr{F}$ au site lisse de $X_{0}$ soit représentable par un $X_{0}$-espace algébrique lisse $F_{0}$.

\section{COHOMOLOGIE ET TORSEURS}

Définition A.3.9 Soient $S$ un schéma, $G$ un $S$-espace algébrique en groupes lisse sur $S$ et $\mathscr{X}$ un $S$-champ algébrique. Soit $p: \mathscr{P} \rightarrow \mathscr{X}$ un 1-morphisme représentable et lisse de $S$-champs algébriques. Une action de $G$ sur $(\mathscr{P}, p)$ est un quadruplet $\left(\mu, \varphi_{\mu}, \varphi_{e}, \varphi_{\text {ass }}\right)$, où $\mu$ est un 1 -morphisme de $G \times_{S} \mathscr{P}$ vers $\mathscr{P}$, et où $\varphi_{\mu}, \varphi_{e}$ et $\varphi_{\text {ass }}$ sont des 2-isomorphismes faisant 2-commuter les diagrammes suivants :
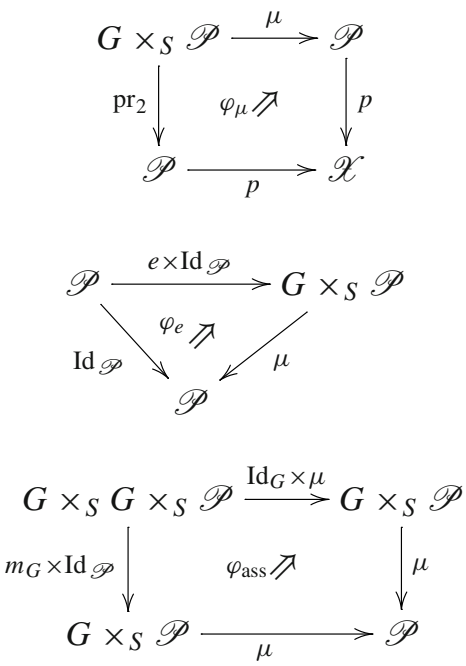

les 2-isomorphismes $\varphi_{e}$ et $\varphi_{\text {ass }}$ étant assujettis aux conditions de compatibilité, que nous nous dispenserons d'écrire, assurant que ce sont des 2-morphismes dans la 2-catégorie des ouverts lisses champêtres de $\mathscr{X}$ (voir juste avant A.1.7).

On dit qu'un tel couple $(\mathscr{P}, p)$ muni d'une action de $G$ est un $G$-torseur sur $\mathscr{X}$ si les deux conditions supplémentaires suivantes sont vérifiées:

(a) $p$ est surjectif;

(b) le morphisme naturel

$$
G \times{ }_{S} \mathscr{P} \longrightarrow \mathscr{P} \times \mathscr{X} \mathscr{P}
$$

induit par le triplet $\left(\mu, \mathrm{pr}_{2}, \varphi_{\mu}\right)$ est un isomorphisme.

La proposition suivante est alors une conséquence des travaux de Giraud dans [23] (voir aussi [15, A.1.10]).

Proposition A.3.10 Soient $S$ un schéma, $G$ un $S$-espace algébrique en groupes commutatifs lisse sur $S$ et $\mathscr{X}$ un $S$-champ algébrique. Alors le groupe $H^{1}(\mathscr{X}, G)$ est canoniquement isomorphe au groupe des classes d'isomorphie de G-torseurs sur $\mathscr{X}$ (muni de la loi de groupe induite par le produit contracté de torseurs, cf. [23] III 2.4.5). 
Remarque A.3.11 Comme d'habitude, un $G$-torseur $p: \mathscr{P} \rightarrow \mathscr{X}$ est trivial si et seulement si le morphisme structural $p$ a une section.

\section{B Cohomologie fppf}

B.1 Sorites sur la cohomologie plate

Soit $\mathscr{X}$ un $S$-champ algébrique. On définit le gros site $f p p f$ de $\mathscr{X}$, noté $\operatorname{fppf}(\mathscr{X})$, de la manière suivante. Les ouverts sont les couples $(U, u)$, où $U$ est un espace algébrique et $u: U \rightarrow \mathscr{X}$ est un morphisme localement de présentation finie. Un morphisme entre deux tels ouverts $(U, u)$ et $(V, v)$ est un couple $(\varphi, \alpha)$ où $\varphi: U \rightarrow V$ est un morphisme d'espaces algébriques et $\alpha$ est un 2-isomorphisme de $u$ vers $v \circ \varphi$. Une famille couvrante est une collection de morphismes $\left(\left(\varphi_{i}, \alpha_{i}\right):\left(U_{i}, u_{i}\right) \rightarrow(U, u)\right)_{i \in I}$ telle que le morphisme

$$
\coprod_{i \in I} \varphi_{i}: \coprod_{i \in I} U_{i} \longrightarrow U
$$

soit fidèlement plat et localement de présentation finie.

Remarque B.1.1 Il est évident que l'on aurait obtenu un topos équivalent en ne prenant pour ouverts que les couples $(U, u)$ où $U$ est un schéma (appliquer le lemme de comparaison de SGA 4, [3] III 4.1). En particulier si $\mathscr{X}$ est un schéma on retrouve le topos des faisceaux sur le gros site fppf usuel (considéré par exemple dans [2], exposé VI, paragraphe 5, p. 124).

On définit de manière évidente les faisceaux d'anneaux $\mathscr{O} \mathscr{X}$ et $\mathbb{Z} \operatorname{sur} \operatorname{fppf}(\mathscr{X})$. On note $\mathscr{X}_{\mathrm{pl}}$ le topos des faisceaux $\operatorname{sur} \operatorname{fppf}(\mathscr{X})$. Si $\mathscr{A}$ est un anneau du topos $\mathscr{X}_{\mathrm{pl}}$, on note $\operatorname{Mod}_{\mathscr{A}}^{\mathrm{pl}}(\mathscr{X})$ la catégorie des faisceaux de modules sur le site annelé $(\operatorname{fppf}(\mathscr{X}), \mathscr{A})$. Elle sera notée $\operatorname{Mod}^{\mathrm{pl}}(\mathscr{X})$ lorsque $\mathscr{A}=\mathscr{O}_{\mathscr{X}}$, et $\mathrm{Ab}^{\mathrm{pl}}(\mathscr{X})$ lorsque $\mathscr{A}=\mathbb{Z}$.

On définit le foncteur «sections globales » en posant pour tout faisceau $\mathscr{F}$ sur $\operatorname{fppf}(\mathscr{X})$ :

$$
\Gamma_{\mathrm{pl}}(\mathscr{X}, \mathscr{F})=\lim _{\longleftarrow} \mathscr{F}(U, u)
$$

où la limite projective est prise sur l'ensemble des couples $(U, u)$ de $\operatorname{fppf}(\mathscr{X})$. Il est clair que ce foncteur commute aux limites projectives quelconques. En particulier il est exact à gauche. De même que dans le cas des faisceaux lisses-étales, il résulte de SGA 4 ([3] II 6.7) que la catégorie $\operatorname{Mod}_{\mathscr{A}}^{\mathrm{pl}}(\mathscr{X})$ est une catégorie abélienne avec suffisament d'objets injectifs. On définit alors $H_{\mathrm{pl}}^{i}(\mathscr{X},$.$) comme étant le i^{i \text { ème }}$ foncteur dérivé à droite de $\Gamma_{\mathrm{pl}}(\mathscr{X},):. \mathrm{Ab}^{\mathrm{pl}}(\mathscr{X}) \rightarrow \mathrm{Ab}$. Il coïncide sur $\operatorname{Mod}_{\mathscr{A}}^{\mathrm{pl}}(\mathscr{X})$ avec le $i^{\text {ième }}$ foncteur dérivé à droite de $\Gamma_{\mathrm{pl}}(\mathscr{X},):. \operatorname{Mod}_{\mathscr{A}}^{\mathrm{pl}}(\mathscr{X}) \rightarrow\left(\Gamma_{\mathrm{pl}}(\mathscr{X}, \mathscr{A})\right.$-Mod $)([4]$ V 3.5). 


\section{FONCTORIALITÉ.}

Soit $f: \mathscr{X} \rightarrow \mathscr{Y}$ un 1-morphisme de $S$-champs algébriques. On lui associe un couple de foncteurs adjoints $\left(f_{\mathrm{pl}}^{-1}, f_{*}^{\mathrm{pl}}\right)$ d'une manière tout à fait analogue à ce qui a été fait pour les faisceaux lisses-étales. Le foncteur $f_{*}^{\mathrm{pl}}$ est alors exact à gauche, et on note $R^{i} f_{*}^{\mathrm{pl}}$ ses foncteurs dérivés à droite.

Remarque B.1.2 Contrairement à ce qu'il se passe dans le cas des faisceaux lissesétales, la limite inductive qui définit le foncteur image inverse $f_{\mathrm{pl}}^{-1}$ est filtrante. Ceci est essentiellement dû au fait qu'un morphisme entre deux objets localement de présentation finie est lui-même localement de présentation finie (ce qui se produisait aussi pour le site étale, mais n'était plus vrai en remplaçant étale par lisse). On en déduit (cf. par exemple [36] annexe A) que le foncteur $f_{\mathrm{pl}}^{-1}$ est exact, et donc que le couple $\left(f_{\mathrm{pl}}^{-1}, f_{*}^{\mathrm{pl}}\right)$ est un morphisme de topos.

Notons ici aussi deux cas particuliers dans lesquels les foncteurs image directe ou image inverse ont une expression plus simple. Lorsque $f$ est localement de présentation finie, le foncteur $f_{\mathrm{pl}}^{-1}$ est simplement le foncteur de restriction au site $f p p f$ de $\mathscr{X}$ via le foncteur $(U, u) \mapsto(U, f \circ u)$. Si $f$ est représentable, le foncteur $f_{*}^{\mathrm{pl}}$ provient d'une application continue $\operatorname{fppf}(\mathscr{X}) \rightarrow \operatorname{fppf}(\mathscr{Y})$, qui à un ouvert fppf $u: U \rightarrow \mathscr{Y}$ associe l'ouvert formé de l'espace algébrique $U \times \mathscr{Y} \mathscr{X}$ muni de la projection sur $\mathscr{X}$. Naturellement ceci n'est pas vrai si $f$ n'est pas représentable, puisqu'alors $U \times \mathscr{Y}$ $\mathscr{X}$ n'est pas nécessairement un espace algébrique. Ce défaut s'avère gênant dans le calcul des images directes supérieures, et motive l'introduction du site fppf champêtre ci-dessous.

\section{LE GROS SITE fppf CHAMPÊTRE D'UN $S$-CHAMP ALGÉBRIQUE.}

Soit $\mathscr{X}$ un $S$-champ algébrique. On définit le gros site fppf champêtre de $\mathscr{X}$ de la manière suivante. Un ouvert de $\mathscr{X}$ est un couple $(\mathscr{U}, u)$ où $\mathscr{U}$ est un $S$-champ algébrique et $u: \mathscr{U} \rightarrow \mathscr{X}$ est un morphisme représentable et localement de présentation finie. Un 1-morphisme de $(\mathscr{U}, u)$ vers $(\mathscr{V}, v)$ est un couple $(\varphi, \alpha)$ où $\varphi: \mathscr{U} \rightarrow \mathscr{V}$ est un 1-morphisme de $S$-champs algébriques et $\alpha$ est un 2-isomorphisme de $u$ dans $v \circ \varphi$. Si $(\varphi, \alpha)$ et $(\psi, \beta)$ sont deux 1-morphismes de $(\mathscr{U}, u)$ dans $(\mathscr{V}, v)$, un 2-morphisme entre $(\varphi, \alpha)$ et $(\psi, \beta)$ est un 2-isomorphisme $\gamma: \varphi \Rightarrow \psi$ tel que $\beta=\left(v_{*} \gamma\right) \circ \alpha$.

L'analogue fppf du lemme A.1.7 est alors valable, de sorte que la 2-catégorie que nous venons de décrire est en fait équivalente à une catégorie, que nous appellerons la catégorie des ouverts fppf champêtres de $\mathscr{X}$. On définit maintenant le gros site fppf champêtre de $\mathscr{X}$ de manière évidente, et on le note $\operatorname{fppfc}(\mathscr{X})$.

Proposition B.1.3 [15, A.2.1.3] Le foncteur d'inclusion $\operatorname{fppf}(\mathscr{X}) \rightarrow \operatorname{fppfc}(\mathscr{X})$ induit une équivalence de topos de la catégorie des faisceaux sur $\operatorname{fppfc}(\mathscr{X})$ vers la catégorie des faisceaux $\operatorname{sur} \operatorname{fppf}(\mathscr{X})$.

On voit alors facilement que via cette équivalence de catégories, le foncteur image directe $f_{*}^{\mathrm{pl}}$ provient d'une application continue

$$
\operatorname{fppfc}(\mathscr{X}) \longrightarrow \operatorname{fppfc}(\mathscr{Y})
$$


qui envoie un ouvert $u: \mathscr{U} \rightarrow \mathscr{Y}$ de $\mathscr{Y}$ sur l'ouvert $\mathscr{X} \times \mathscr{Y} \mathscr{U} \rightarrow \mathscr{X}$ de $\mathscr{X}$.

Proposition B.1.4 [15, A.2.1.4] Soit $f: \mathscr{X} \rightarrow \mathscr{Y}$ un morphisme de S-champs algébriques.

1) Soit $\mathscr{F}$ un faisceau abélien sur $\operatorname{fppf}(\mathscr{X})$. Alors le faisceau $R^{q} f_{*}^{\mathrm{pl}} \mathscr{F}$ est le faisceau associé au préfaisceau qui à tout ouvert fppf $u: U \rightarrow \mathscr{Y}$ de $\mathscr{Y}$ associe $H_{\mathrm{pl}}^{q}\left(\mathscr{X} \times \mathscr{Y} U, \mathscr{F}\left(\mathscr{X} \times \mathscr{Y} U, \mathrm{pr}_{\mathscr{X}}\right)\right.$.

2) La restriction du foncteur $R^{q} f_{*}^{\mathrm{pl}}$ à la catégorie $\operatorname{Mod}^{\mathrm{pl}}(\mathscr{X})$ cö̈ncide avec le $q^{i e ̀ m e}$ foncteur dérivé à droite du foncteur $f_{*}^{\mathrm{pl}}: \operatorname{Mod}^{\mathrm{pl}}(\mathscr{X}) \rightarrow \operatorname{Mod}^{\mathrm{pl}}(\mathscr{Y})$.

B.2 Comparaison avec la cohomologie lisse-étale

Soit $\mathscr{X}$ un $S$-champ algébrique. On a une application continue évidente:

$$
p: \operatorname{fppf}(\mathscr{X}) \longrightarrow \operatorname{Lis-ét}(\mathscr{X})
$$

induite par le foncteur d'inclusion de Lis-ét $(\mathscr{X})$ dans $\operatorname{fppf}(\mathscr{X})$. En particulier $p$ induit un couple de foncteurs adjoints :

$$
\begin{aligned}
p_{*}: \mathscr{X}_{\mathrm{pl}} \longrightarrow \mathscr{X}_{\text {lis-êt }} \\
p^{-1}: \mathscr{X}_{\text {lis-ét }} \longrightarrow \mathscr{X}_{\mathrm{pl}} .
\end{aligned}
$$

Le foncteur $p^{-1}$ peut être décrit de la manière suivante. On définit d'abord un adjoint à gauche au foncteur $p_{*}$ pour les préfaisceaux, que l'on note $\widehat{p^{-1}}$, en associant à tout préfaisceau $\mathscr{F}$ sur Lis-ét $(\mathscr{X})$ le préfaisceau $\operatorname{sur} \operatorname{fppf}(\mathscr{X})$ qui à tout ouvert fppf $u: U \rightarrow \mathscr{X}$ associe

$$
\left.\widehat{\left(p^{-1} \mathscr{F}\right.}\right)(U, u)=\underline{\lim } \mathscr{F}(V, v)
$$

où la limite inductive est prise sur l'ensemble des diagrammes 2-commutatifs :

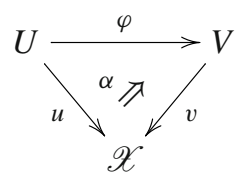

avec $(V, v) \in \operatorname{obLis-ét}(\mathscr{X})$. On définit alors $p^{-1}$ en posant $p^{-1}=\widehat{a} \underline{p}^{-1}$ où $\underline{a}$ est le foncteur «faisceau associé ». Encore une fois, on voit que $\widehat{p^{-1}}$ est défini par une limite inductive sur Lis-ét $(\mathscr{X})$ qui n'est pas filtrante. Le couple $\left(p^{-1}, p_{*}\right)$ n'est donc pas un morphisme de topos a priori, et il n'y a aucune raison formelle pour que $p_{*}$ transforme les objets injectifs de $\mathrm{Ab}^{\mathrm{pl}}(\mathscr{X})$ en injectifs de $\mathrm{Ab}(\mathscr{X})$, ce qui nous oblige à travailler un peu plus pour obtenir la suite spectrale B.2.3 ci-dessous. 
Lemme B.2.1 $[15, \mathrm{~A} .2 .2 .1]$ Si $\mathscr{F}$ est un objet injectif de $\mathrm{Ab}^{\mathrm{pl}}(\mathscr{X})$, alors $p_{*} \mathscr{F}$ est un faisceau acyclique pour le foncteur $\Gamma_{\text {lis-ét }}(\mathscr{X},$.$) .$

Lemme B.2.2 [15, A.2.2.2] Le morphisme naturel

$$
\Gamma_{\mathrm{pl}}(\mathscr{X}, \mathscr{F}) \longrightarrow \Gamma_{\text {lis-ét }}\left(\mathscr{X}, p_{*} \mathscr{F}\right)
$$

est un isomorphisme.

On déduit des deux résultats précédents une suite spectrale de Leray, qui résume les relations générales entre cohomologie lisse-étale et cohomologie fppf.

Théorème B.2.3 [15, A.2.2.3] Soit $\mathscr{X}$ un S-champ algébrique, et soit $\mathscr{F}$ un faisceau abélien sur $\operatorname{fppf}(\mathscr{X})$. On a alors une suite spectrale (fonctorielle en $\mathscr{F}$ ):

$$
H_{\text {lis-êt }}^{p}\left(\mathscr{X}, R^{q} p_{*} \mathscr{F}\right) \Rightarrow H_{\mathrm{pl}}^{p+q}(\mathscr{X}, \mathscr{F}) \text {. }
$$

Remarque B.2.4 On en déduit en particulier des morphismes canoniques :

$$
H_{\text {lis-ét }}^{p}\left(\mathscr{X}, p_{*} \mathscr{F}\right) \longrightarrow H_{\mathrm{pl}}^{p}(\mathscr{X}, \mathscr{F}) \text {. }
$$

De plus ces morphismes sont des isomorphismes si pour tout $q>0$, le faisceau lisse-étale $R^{q} p_{*} \mathscr{F}$ est nul.

Nous allons à présent généraliser aux champs algébriques le résultat de Grothendieck ([2], exposé VI, paragraphe 11) selon lequel si $G$ est un groupe lisse sur un schéma $X$, le morphisme canonique

$$
H_{\text {ét }}^{q}\left(X, p_{*} G\right) \longrightarrow H_{\mathrm{pl}}^{q}(X, G)
$$

est un isomorphisme pour tout $q$. Dans la mesure où nous n'utiliserons ce résultat que pour le groupe $\mathbb{G}_{\mathrm{m}}$, nous n'avons pas cherché à le démontrer pour les «champs en groupes » lisses sur $\mathscr{X}$ (notion qui resterait d'ailleurs à définir), mais nous nous sommes contenté de considérer un groupe lisse sur la base $S$. Dans ce cadre élémentaire, le résultat se déduit assez facilement du cas des schémas. En effet pour démontrer B.2.5, il suffit d'après B.2.4 de montrer que pour tout $q>0$, le faisceau $R^{q} p_{*} G$ est nul. Or ce faisceau est le faisceau lisse-étale associé au préfaisceau qui à tout ouvert $u: U \rightarrow \mathscr{X}$ associe $H_{\mathrm{pl}}^{q}(U, G)$. Il suffit donc de montrer que pour tout $\xi \in H_{\mathrm{pl}}^{q}(U, G)$, il existe un morphisme étale et surjectif $U^{\prime} \rightarrow U$ tel que $\xi_{\left.\right|_{U^{\prime}}}$ soit nul. Cette dernière question ne fait intervenir que des schémas ( $c f$. [15] pour plus de détails).

Théorème B.2.5 [15, A.2.2.5] Soient $S$ un schéma, $G$ un schéma en groupes lisse sur $S$, et $\mathscr{X}$ un $S$-champ algébrique. On note $f$ le morphisme de $\mathscr{X}$ dans $S$, et l'on note encore $G$ le faisceau défini sur le site $\operatorname{fppf}(\mathscr{X})$ par:

$$
\forall(U, u) \in \operatorname{ob} \operatorname{fppf}(\mathscr{X}) \quad G(U, u):=G(U, f \circ u)=\operatorname{Hom}_{S}(U, G) .
$$




\section{Alors pour tout q le morphisme canonique}

$$
H_{\text {lis-ét }}^{q}\left(\mathscr{X}, p_{*} G\right) \longrightarrow H_{\mathrm{pl}}^{q}(\mathscr{X}, G)
$$

\section{est un isomorphisme.}

Open Access This article is distributed under the terms of the Creative Commons Attribution Noncommercial License which permits any noncommercial use, distribution, and reproduction in any medium, provided the original author(s) and source are credited.

\section{Références}

1. Schémas en groupes. II : Groupes de type multiplicatif, et structure des schémas en groupes généraux. Séminaire de Géométrie Algébrique du Bois Marie 1962/64 (SGA 3). Dirigé par M. Demazure et A. Grothendieck. Lecture Notes in Mathematics, vol. 152. Springer, Berlin (1962/1964)

2. Dix exposés sur la cohomologie des schémas. Advanced Studies in Pure Mathematics, vol. 3. NorthHolland Publishing Co., Amsterdam, 1968

3. Théorie des topos et cohomologie étale des schémas. Tome 1 : Théorie des topos. Springer, Berlin (1972). Séminaire de Géométrie Algébrique du Bois-Marie 1963-1964 (SGA 4), Dirigé par M. Artin, A. Grothendieck, et J. L. Verdier. Avec la collaboration de N. Bourbaki, P. Deligne et B. Saint-Donat. Lecture Notes in Mathematics, vol. 269

4. Théorie des topos et cohomologie étale des schémas. Tome 2. Springer, Berlin (1972). Séminaire de Géométrie Algébrique du Bois-Marie 1963-1964 (SGA 4), Dirigé par M. Artin, A. Grothendieck et J. L. Verdier. Avec la collaboration de N. Bourbaki, P. Deligne et B. Saint-Donat, Lecture Notes in Mathematics, vol. 270

5. Revêtements étales et groupe fondamental (SGA 1). Documents Mathématiques (Paris), 3. Société Mathématique de France, Paris, 2003. Séminaire de géométrie algébrique du Bois Marie 1960-61. Dirigé par A. Grothendieck, augmenté de deux exposés de Mme M. Raynaud. Édition recomposée et annotée du volume 224 des Lectures Notes in Mathematics publiée en 1971 par Springer-Verlag

6. Abramovich, D. et Vistoli, A. : Complete moduli for families over semistable curves. http://fr.arxiv. org/abs/math/9811059, 1998

7. Abramovich, D. et Vistoli, A. : Complete moduli for fibered surfaces. In : Recent progress in intersection theory (Bologna, 1997), Trends Math., pp. 1-31. Birkhäuser Boston, Boston (2000)

8. Abramovich, D. et Vistoli, A. : Compactifying the space of stable maps. J. Am. Math. Soc. 15(1), 27-75 (electronic) (2002)

9. Aoki, M.: Deformation theory of algebraic stacks. Compos. Math. 141(1), 19-34 (2005)

10. Aoki, M.: Erratum : "Hom stacks". Manuscripta Math. 121(1), 135 (2006)

11. Aoki, M.: Hom stacks. Manuscripta Math. 119(1), 37-56 (2006)

12. Artin, M. : Algebraization of formal moduli. I. In : Global Analysis (Papers in Honor of K. Kodaira), pp. 21-71. Univ. Tokyo Press, Tokyo (1969)

13. Artin, M.: Versal deformations and algebraic stacks. Invent. Math. 27, 165-189 (1974)

14. Bosch, S., Lütkebohmert, W., Raynaud, M. : Néron models. de Ergebnisse der Mathematik und ihrer Grenzgebiete (3) [Results in Mathematics and Related Areas (3)], vol. 21. Springer, Berlin (1990)

15. Brochard, S. : Champs algébriques et foncteur de Picard. Thèse de l'université de Rennes 1 , math.AG/ 0808.3253 (2007)

16. Cadman, C.: Using stacks to impose tangency conditions on curves. Am. J. Math. 129(2), 405-427 (2007)

17. Chiodo, A. : Stable twisted curves and their $r$-spin structures. À paraître dans les Annales de l'Institut Fourier. math.AG/0603687

18. Conrad, B.: A modern proof of Chevalley's theorem on algebraic groups. J. Ramanujan Math. Soc. 17(1), 1-18 (2002)

19. Deligne, P. : Théorème de Lefschetz et critères de dégénérescence de suites spectrales. Inst. Hautes Études Sci. Publ. Math. 35, 259-278 (1968)

20. Faltings, G.: Finiteness of coherent cohomology for proper fppf stacks. J. Algebraic Geom. 12(2), 357-366 (2003) 
21. Ferrand, D.: Conducteur, descente et pincement. Bull. Soc. Math. France 131(4), 553-585 (2003)

22. Fulton, W. et Olsson, M.C. : The Picard group of $\mathrm{M}_{1,1}$. arXiv:0704.2214

23. Giraud, J. : Cohomologie non abélienne. Springer-Verlag, Berlin, 1971. Die Grundlehren der mathematischen Wissenschaften, Band 179

24. Grothendieck, A.: Sur quelques points d'algèbre homologique. Tôhoku Math. J. (2) 9, 119-221 (1957)

25. Grothendieck, A. : Éléments de géométrie algébrique. II. Étude globale élémentaire de quelques classes de morphismes. Inst. Hautes Études Sci. Publ. Math. 8, 222 (1961)

26. Grothendieck, A. : Éléments de géométrie algébrique. III. Étude cohomologique des faisceaux cohérents. I. Inst. Hautes Études Sci. Publ. Math. 11, 167 (1961)

27. Grothendieck, A. : Éléments de géométrie algébrique. IV. Étude locale des schémas et des morphismes de schémas. I. Inst. Hautes Études Sci. Publ. Math. 20, 259 (1964)

28. Grothendieck, A. : Éléments de géométrie algébrique. IV. Étude locale des schémas et des morphismes de schémas. II. Inst. Hautes Études Sci. Publ. Math. 24, 231 (1965)

29. Grothendieck, A. : Éléments de géométrie algébrique. IV. Étude locale des schémas et des morphismes de schémas. III. Inst. Hautes Études Sci. Publ. Math. 28, 255 (1966)

30. Illusie, L. : Complexe cotangent et déformations. I. Lecture Notes in Mathematics, vol. 239. Springer, Berlin (1971)

31. Kleiman, S.L. : The Picard scheme. In Fundamental algebraic geometry, de Math. Surveys Monogr., vol. 123, pp. 235-321. American Mathematical Society, Providence (2005)

32. Knutson, D. : Algebraic spaces. Lecture Notes in Mathematics, vol. 203. Springer, Berlin (1971)

33. Laszlo, Y. et Olsson, M.C. : The six operations for sheaves on artin stacks i : Finite coefficients. À paraître dans Publ. Math. IHES. http://www.math.polytechnique.fr/ laszlo/

34. Laumon, G. et Moret-Bailly, L. : Champs algébriques, volume 39 de Ergebnisse der Mathematik und ihrer Grenzgebiete. 3. Folge. A Series of Modern Surveys in Mathematics [Results in Mathematics and Related Areas. 3rd Series. A Series of Modern Surveys in Mathematics]. Springer, Berlin (2000)

35. Lieblich, M.: Moduli of twisted sheaves. Duke Math. J. 138(1), 23-118 (2007)

36. Milne, J.S.: Étale cohomology, de Princeton Mathematical Series, vol. 33. Princeton University Press, Princeton (1980)

37. Mumford, D.: Picard groups of moduli problems. In : Arithmetical Algebraic Geometry (Proc. Conf. Purdue Univ., 1963), pp. 33-81. Harper \& Row, New York (1965)

38. Mumford, D.: Abelian varieties. Tata Institute of Fundamental Research Studies in Mathematics, No. 5. Published for the Tata Institute of Fundamental Research, Bombay (1970)

39. Noohi, B.: Picard stack of a weighted projective stack. http://www.math.fsu.edu/ noohi/papers/ PicardSt.pdf

40. Olsson, M.: Sheaves on Artin stacks. J. Reine Angew. Math. 603, 55-112 (2007)

41. Olsson, M.C.: On proper coverings of Artin stacks. Adv. Math. 198(1), 93-106 (2005)

42. Olsson, M.C.: Deformation theory of representable morphisms of algebraic stacks. Math. Z. 253(1), 25-62 (2006)

43. Olsson, M.C.: (Log) twisted curves. Compos. Math. 143(2), 476-494 (2007)

44. Springer, T.A. : Linear algebraic groups, de Progress in Mathematics, vol. 9, 2nd edn . Birkhäuser Boston Inc., Boston (1998) 